\title{
Saprophytic Colonization and Sporulation of Virulent and Hypovirulent Cryphonectria parasitica on American Chestnut (Castanea dentata) and Scarlet Oak (Quercus coccinea)
}

Eric Shelton Goddard

Follow this and additional works at: https://researchrepository.wvu.edu/etd

\section{Recommended Citation}

Goddard, Eric Shelton, "Saprophytic Colonization and Sporulation of Virulent and Hypovirulent Cryphonectria parasitica on American Chestnut (Castanea dentata) and Scarlet Oak (Quercus coccinea)" (2015). Graduate Theses, Dissertations, and Problem Reports. 5686.

https://researchrepository.wvu.edu/etd/5686

This Thesis is protected by copyright and/or related rights. It has been brought to you by the The Research Repository @ WVU with permission from the rights-holder(s). You are free to use this Thesis in any way that is permitted by the copyright and related rights legislation that applies to your use. For other uses you must obtain permission from the rights-holder(s) directly, unless additional rights are indicated by a Creative Commons license in the record and/ or on the work itself. This Thesis has been accepted for inclusion in WVU Graduate Theses, Dissertations, and Problem Reports collection by an authorized administrator of The Research Repository @ WVU. For more information, please contact researchrepository@mail.wvu.edu. 


\title{
Saprophytic Colonization and Sporulation of Virulent and Hypovirulent Cryphonectria parasitica on American Chestnut (Castanea dentata) and Scarlet Oak (Quercus coccinea)
}

\section{Eric Shelton Goddard}

Thesis submitted to the

Davis College of Agriculture, Natural Resources and Design

at West Virginia University

In partial fulfillment of the requirements for the degree of:

\section{Master of Science \\ in \\ Plant Pathology}

\author{
William L. MacDonald, Ph.D., Chair \\ Daniel G. Panaccione, Ph.D. \\ John R. Brooks, Ph.D.
}

Division of Plant and Soil Sciences

Morgantown, WV

2015

Keywords: chestnut blight, hypovirulence, saprophytic fungi 


\title{
ABSTRACT \\ Saprophytic Colonization and Sporulation of Virulent and Hypovirulent Cryphonectria parasitica on American Chestnut (Castanea dentata) and Scarlet Oak (Quercus coccinea)
}

\author{
Eric S. Goddard
}

Colonization and sporulation (stroma production) of virulent (V) and hypovirulent (HV) Cryphonectria parasitica, were evaluated on two hosts to better understand the saprophytic stage of the fungus and its ability to produce $\mathrm{HV}$ inoculum. Both the $\mathrm{V}$ and HV C. parasitica strains were isolated from an existing chestnut orchard at the test site. Castanea dentata (American chestnut) and Quercus coccinea (scarlet oak) were used as test hosts. Sixty centimeter long stems of both species were cut from saplings and placed as pairs in three-layer, triangular stacks. Each stack was wound inoculated with either V, $\mathrm{HV}$ or water agar (control) inoculum. Five groups of three inoculated stacks were placed on a wooded, upper-slope terrace at the Bunner's Ridge, WV experimental chestnut site. The stems were cut the week of May $15^{\text {th }}, 2011$ and three inoculations were made starting on May $20^{\text {th }}$ [Inoculation Period-1 (IP-1)], August $4^{\text {th }}$ (IP-2) and October $4^{\text {th }}$ (IP-3).

Lesions resulting from each IP was measured for colonization in $\mathrm{cm}^{2}$ from the point of inoculation and the colonized area was visually ranked for sporulation. The infected area was then sampled for fungi at monthly intervals following the date of inoculation until December $8^{\text {th }}, 2011$. The total colonization and stroma production were analyzed along with the effect of stack layers and stack placement at the site. Results indicated that colonization and sporulation of $C$. parasitica generally were not significantly different between $C$. dentata and $Q$. coccinea and declined propotionally with time for each subsequent inoculation period. With each successive IP, the area $C$. parasitica was able to colonize decreased, while the colonization and recovery of other fungi increased. Though V grew and sporulated significantly more than HV for IP-1 on both hosts, the differences were not significant for IP's 2 and 3 . The analysis of layer and location effects did not conclusively indicate trends that better colonization or sporulation for any specific layer or group of stacks occurred. Isolations showed that $\mathrm{HV}$ isolates were able to occasionally colonize $\mathrm{V}$ and Control piles and that non-inoculated stems became naturally HV infected up to six months after the initial inoculations. Results also indicate that $\mathrm{V}$ and $\mathrm{HV}$ are able to be successfully inoculated up to four months following the death of their host. However, colonization during successive IP's was greatly diminished when compared to IP-1. V and HV colonized and sporulated similarly to each other on both hosts and better, but generally not significantly so, on scarlet oak. Also, HV competed nearly as well as $\mathrm{V}$ as a saprophyte on both hosts. Initial colonization during IP-1 may have occurred readily and maintained a high recovery rate for $\mathrm{V}$ and $\mathrm{HV}$ because stems were cut and active host resistance was eliminated. Also, colonization and recovery of $C$. parasitica during subsequent IP's clearly was diminished by the aggressive colonization by other organisms that accompanied bark deterioration. The time of year stems were cut and bark thickness also may have played important roles in the results. 


\section{ACKNOWLEDGEMENTS}

There were many that helped in support and completion of this thesis. Without their support, none of this would have been possible. From the administration within the Davis College, the members of my committee, many professors, fellow classmates, Doug Six at Blue Mtn Inc, and the friends and family that supported, helped and taught me the skills and knowledge required for success, I owe my eternal gratitude. Also, none of this would have been possible without the unwaning patience and guidance of my advisor Dr. William MacDonald throughout this research project. Special thanks go out to Mark Double and Dr. Daniel Panaccione, you both taught me more than you know and were always there when I asked. Mark and Dr. Dan's knowledge and assistance with complex lab procedures and willingness to help was critical to my success. Also, my thanks and appreciation goes out to Dr. John Brooks for being on this graduate committee and aiding whenever called upon. I am forever grateful to everyone that was there when needed for advice, friendship and aid in this difficult journey, you know who you are.

Thanks Dr. Mac 


\section{TABLE OF CONTENTS}

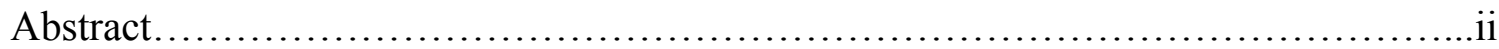

Acknowledgements........................................................



Research Objective........................................................vi

Introduction and Literature Review............................................

Methods and Materials............................................................ 11

Study Location....................................................... 11

Cryphonectria parasitica Strains Selected...............................11

Pathogenicity Tests................................................... 13

dsRNA Extraction........................................................ 14

Vegetative Compatibility........................................... 15

Stem Origination, Labeling and Selection................................ 15

Stacks............................................................... 16

Inoculum Production and Inoculation Procedure............................ 17

Time line for canker colonization measurements and sporulation evaluation......18



Culture and Single Spore Procedures...................................21

Fungi other than $C$. parasitica .......................................22

Dissemination of HV strains..........................................22

Sporulation Ranking..............................................23

Statistics.........................................................28



Chapter 1 Colonization................................................29

Section 1: Analysis of Total Colonization................................29

Section 2: Effect of Layers on Colonization.................................. 36

Section 3: Effect of Stack Location on Colonization..........................41



Chapter 2 - Sporulation............................................. 47

Section 1: Analysis of Total Sporulation..................................47



Section 3: Effect of Stack Location on Sporulation .........................58

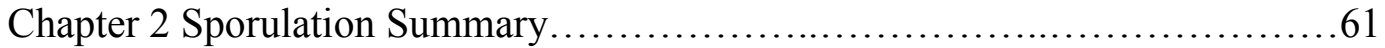


Chapter 3 - Fungi Associated with Inoculated Stems .........................63

Section 1: Castanea dentata V Inoculation ...............................66

Section 2: Castanea dentata HV Inoculation ..............................66

Section 3: Castanea dentata Water Agar (Control) Inoculation .................69

Section 4: Quercus coccinea V Inoculation .................................71

Section 5: Quercus coccinea HV Inoculation ..............................74

Section 6: Quercus coccinea Water Agar (Control) Inoculation ................76

Section 7: C. parasitica Isolated External to Observable Infection..............79



Colonization and Sporulation......................................... 82

Other Organisms................................................ 84

Tree Hosts..................................................... 87

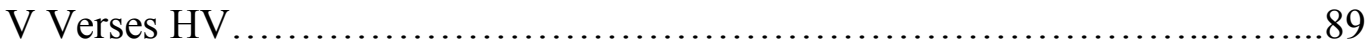



Conclusions................................................... 90

Future Direction for Experimentation..................................91

Appendix A.............................................................. 93

Media Ingredients.............................................. 93

Potato Dextrose Agar..........................................93

Glucose Yeast Extract with Antibiotics............................93

Modified Brome-Cresol Green Medium..............................93

Stack Diagram.................................................... 93

Additional Photos....................................................94

Other Fungi Isolated............................................96

Appendix B: PCR Products and DNA Sequence Results of Other Isolated Fungi........98






\section{RESEARCH OBJECTIVE}

The objective of this research thesis was to evaluate the potential of hypovirulent (HV) and virulent (V) strains of Cryphonectria parasitica to colonize and sporulate (produce stroma) on stacked, dead stems of American chestnut (Castanea dentata) and scarlet oak (Quercus coccinea). Specifically, the following were evaluated:

1) The ability of V and HV strains to colonize and sporulate on bark of artificially killed American chestnut and scarlet oak stems;

2) the influence of the date of inoculation after cutting on colonization and sporulation;

3) the colonization of the stems by other saprophytic fungi; and,

4) dissemination of the HV strain to non-HV inoculated sites among the cut stems. 


\section{INTRODUCTION AND LITERATURE REVIEW}

The heart of the American chestnut's (Castanea dentata Marsh. Borkh.) native range could be found in the Appalachian Mountains. This once valuable hardwood species played host to one the most destructive forest pathogens of the $20^{\text {th }}$ century. The pathogen, Cryphonectria parasitica (Murr.) Barr., was introduced from Asia via trade during the late 1800's (Anagnostakis, 1987; Milgroom, 1992). Chestnut blight disease symptoms were first observed in the New York Zoological Garden in 1904 (Alexopoulos et al., 1996). Both economically and ecologically, the American chestnut was reduced from a dominant species within eastern North America at a great price. At the time of the introduction of the pathogen, on average $25 \%$ or more of the upland canopy, ranging from Georgia through Maine and into southeastern Canada, was comprised of chestnut (Braun, 1950). Many chestnuts were giants having an average life span of 400 years. A mature tree could grow to a miraculous seven feet in diameter and 120 feet in height (Saucier, 1945). The tree produced shelter and mast for foraging animals. The nuts were considered delectable by humans and had substantial economic value (West 1988).

Furthermore, the wood was decay resistant, flexible, and provided valuable tannins to the leather industry. The straight grain and aesthetic quality of the wood made it a valuable timber resource (Kuhlman, 1978).

Following the onset of chestnut blight, the 50 years that followed were devastating for the species, ecosystem and economy. The result was the loss of over 3.6 million hectares of chestnut forests in West Virginia, Pennsylvania, and North Carolina with an estimated 1912 economic loss of 82.5 million dollars (Anagnostakis, 1987). This once dominant "redwood of the east" was diminished to an understory shrub (Alexopoulos et al., 1996). Fortunately, the species has survived due to its tenacious ability to sprout. The species now exists mostly as sprouts, saplings and small trees that 
rarely reach more than 25 feet in height (Stephenson, et al., 1991). New sprouts begin as epicormic buds. Chestnut produces these in abundance, particularly when stressed. The new sprouts grow until they also are infected. Sprouts can remain healthy for several years and sometimes can reach small tree size because they escape infection. Once the fungus infects the tree, it girdles vascular tissue. The vegetation beyond the point of infection eventually wilts and necrosis follows due to lack of water flow and nutrient exchange (Alexopoulos et al., 1996).

The chestnut blight fungus was originally named Diaporthe parasitica Murr. (Murrill, 1906). In 1978, the genus underwent another revision and was given its current name as Cryphonectria parasitica, "the hidden nectria" (Newhouse, 1978). It is a member of Ascomycota within the order Diaporthales. It forms orange stroma containing perithecial ascocarps in its perfect stage and pycnidia in its imperfect stage (Alexopoulos et al., 1996). The ascospores are two celled and can contain one-to-four nuclei per cell. They are not sticky and are disseminated primarily by wind and rain. The conidia are single-celled, curved, minute, and generally are formed in mucilaginous tendrils called cirrhi. These sticky spores are disseminated readily by insects, birds, mammals and wind-splashed rain. Conidia are produced in great numbers from diseased bark (Alexopoulos et al., 1996).

This species is homothallic but preferentially outcrosses when possible (Puhalla and Anagnostakis, 1971). This was determined by culturing genetically marked axenically grown mycelia from single uninucleate conidia. Chestnut trees were then inoculated with the individual mycelial colonies. The resulting cankers reproduced sexually and formed ascospores. Wild-type natural populations also need to be considered. Several C. parasitica populations from the USA, Europe and Asia demonstrated non-preferential selfing or outcrossing in a single stand leading to a mixed 
mating type population. Reasons remain unclear but genetic, demographic, and ecological factors likely contribute to homothallic and idiomorphic mixed population dynamics (Marra, et al., 2004). However, laboratory attempts at sexual reproduction reveal the fungus tends to self and sexual reproduction has not been demonstrated on agar media but has been demonstrated on bark (Anagnostakis, 1977). The conditions for perithecial formation to occur in culture are unknown.

Cryphonectria parasitica has been described as a wound pathogen. Spores are disseminated primarily by wind and rain. Insects also can carry inoculum and cause wounds through which conidia can enter (Alexopoulos et. al., 1996). Wound infections occur on saplings to mature trees. Prolific sprouting also creates many wounds from included bark (Shigo, 1986). These bark inclusions create an ideal infection court on young, otherwise unwounded sprouts and saplings. C. parasitica does not appear to infect the underground root system of trees and infections are not known to spread from root to sprouts. However, infected root collars can result in sprout infection (Stilwell, et al., 2003). Ascospores, conidia, or hyphae must be present to begin a new infection (Shigo, 1986; Alexopoulos et. al., 1996). Once infection occurs, complete girdling of the stem will follow, usually within a season or two. This will eventually lead to death of the tree.

Cryphonectria parasitica is known as a facultative parasite as it functions saprophytically in some phases of its lifecycle (Newhouse, 1990). The parasitic process begins when the fungus establishes within a wound. The germinating hyphae develop a mycelial fan underneath the bark surface. The trees response is to try to compartmentalize the wound and contain the infection (Shigo et al., 1977). Attempting to slow the invading organism, the host response is a release of a cocktail of inhibitory compounds, and extractives such as; saponin, catechol, terpinoids, phenolic compounds 
and tannic acids (McCarroll and Thor, 1985). A wound periderm barrier also is formed that may limit the growth of individual hyphae (Griffin, 1986). However, the advancing mycelium overtakes the host response and leads to canker formation. The canker formation occurs as the advancing mycelium penetrates this compartmentalizing barrier before it is fully formed (Griffin, 1986). The fungus releases a combination of enzymes and organic acids that degrade and digest the cells of the vascular phloem and cambium (Welch et al., 2007). A depressed or sunken canker forms due to the death of vascular tissue. As the canker fully girdles the stem, wilting and necrosis beyond the infection quickly follows.

Healthy wound or infection response by the tree produces callus tissue that builds up as successive layers of new wood. Callus is formed by the cambium to seal off the wound from the environment and contain or suppress the invading organism(s) (Shigo, 1977). This type of wound response is evident in Asiatic chestnuts that express resistance to C. parasitica (Shear et al., 1917).

Formation of healthy callus in response to wounding and infection also is evident under a different set of circumstances. The European chestnut (Castanea sativa Mill.) also is affected by C. parasitica and the disease epidemic. However, in the 1950's a plant pathologist by the name of Antonio Biraghi found trees living with the blight that formed healthy callus tissue in response to infection (Biraghi, 1953; MacDonald, 1985). Jean Grente, a French mycologist, then cultured the fungi from these cankers. The $C$. parasitica fungus was present, but it was restricted to the outer bark (Grente, 1978). This discovery lead to a break-though, and Grente eventually would demonstrate the potential use as a biological control agent. The new fungal isolate had new phenotypic characters not expressed in virulent forms. 
Grente noticed a different morphology within this apparent "new strain". It was white in culture whereas the virulent strain typically is pigmented orange. These strains sporulated less and their mycelial fan growth in infected trees progressed at a reduced rate resulting in a reduction of virulence (V). He coined the term "hypovirulence" (HV) because of their reduced ability to infect bark. The HV fungus expresses reduced virulence allowing trees to tolerate infections and survive. Now it is known that this effect is the result of a cytoplasmic double-stranded RNA (Anagnostakis, 1987). This dsRNA is now properly named a hypovirus (Choi and Nuss, 1992). Some of the viral effects on the fungal metabolism observed are reduction of surface proteins, conidia production, laccases, cutinase, and reduced accumulation of oxalate (Nuss and Koltkin, 1990). C. parasitica, as well as many saprophytic fungi, produces excessive amounts of this acid to degrade cellulose (Havir and Anagnostakis, 1983). Oxalic oxidase, produced by the tree, creates hydrogen peroxide in the presence of oxalic acid and induces lignification of cell walls and forms callus tissue (Welch et al., 2007). Therefore, the reduced production of oxalic acid helps contribute to callus formation when bark is infected by hypovirulent $C$. parasitica infected chestnut (Havir and Anagnostakis, 1983). The reduced virulence of the fungus allows the tree to respond by producing callus. However, dissemination of hypovirus is dependent upon conidia. Unfortunately, hypovirus infection reduces conidia production, an undesired effect for utilization of these HV strains as biocontrol agents (Nuss, 2005).

Vegetative compatibility (VC) is another issue of concern for dissemination of the hypovirus. When strains are vegetatively compatible anastomosis can occur. Anastomosis is the cytoplasmic fusion and exchange of material within fungi (Anagnostakis and Wagner, 1981). This process allows for the transference of dsRNA hypovirus through cytoplasm (Nuss and Koltkin, 1990). There are approximately 128 
VC types in North America (MacDonald and Fulbright, 1991). Anastomosis is not possible between strains without similar VC genes and vegetative compatibility is regulated by at least six vic loci (McGuire et al., 2005). The hypovirus rarely is capable of being transmitted between incompatible strains. Hypovirus transmission occasionally occurs by the incidental passing through cytoplasm before cell death forms the zone of demarcation between two incompatible fungal colonies. However, hypoviruses are generally transmitted $100 \%$ of the time between completely compatible strains (Robin et al., 2009). VC is an important factor to consider when attempting to use hypovirulent strains of $C$. parasitica as biological control agents. If a hypovirus containing strain is intentionally utilized as a biocontrol agent, the spread would be enhanced if it is compatible with the resident virulent fungi. The hypovirus transmission factors described appear to function on a live or dead host.

The saprophytic colonization of C. parasitica was assessed by Prospero et al., (2006). They studied the transmission of hypovirus in southern Switzerland. Stacked piles of dead European chestnut (Castanea sativa) were utilized. The study took place in coppice (raised from stump sprouts) stands of chestnut with naturally occurring hypovirus $\mathrm{CHV}-1$ and virulent strains of $C$. parasitica. The natural spread of HV strains has been successful in this region. Vegetative compatibility appears to be less restrictive to the transmission of hypovirus in this area of Europe, where as few as $33 \mathrm{vc}$ groups have been identified and some regions support just one or two dominant ve groups (Heiniger and Rigling, 1994). Some individual locations in North America could contain as many as $48 \mathrm{VC}$ types (MacDonald and Fulbright, 1991). This is an important consideration relative to the intentional or unintentional spread of the hypovirus.

Prospero's study (2006) demonstrated the importance of saprophytic colonization of virulent and hypovirulent strains. Comparisons were made between $\mathrm{V}$ and $\mathrm{HV} C$. 
parasitica strains for sporulation and canker growth. The trees utilized were already exhibiting V and HV cankers and canker free trees were used as control stems. Canker colonization and sporulation were measured on live trees and cut and stacked stems. In all accounts, cut and stacked stems had significantly more stroma and colonization on the bark than living stems. In fact, from March, 1996 to April 1997 stroma on dead stems increased two fold but decreased on the living trees. Sporulation also increased on the dead stems but remained low on living stems. Dead stems that originally were infected or showed little sign of infection produced more $\mathrm{V}$ and $\mathrm{HV}$ isolates than living stems. Stroma producing perithecia and pycnidia also formed on stems from virulent and hypovirulent strains. When cultured, a significant amount of conidia was produced from HV colonies. However, in no instance were HV containing cultures recovered from ascospores. Inactive cankers upon stems that originally were living resumed growth and colonized new bark when cut. New stroma also formed in bark of stacks that contained previously uninfected wood. The results showed that between 5 to $41 \%$ (mean 26\%) of the pycnidia examined had conidia containing hypovirus. Single spore counts showed pycnidia from $\mathrm{HV}$ colonies contained $69 \%$ hypovirus containing conidia. New infection transmission rates averaged $18 \%$ for saprophytically produced colonies infected with hypovirus. The results of this study indicated that it is possible to infect and allow for natural dissemination of hypovirus-infected conidia. This research provides further information that saprophytic HV inoculum can contribute to biological control (Prospero et al., 2006).

Cryphonectria parasitica also infects many other chestnut substrates. The fungus can grow in these niches both parasitically and saprophytically (Baird, 1991). Saprophytically, the fungus will grow on leaves, twigs, and burs of American chestnut. Saprophytic colonization also was observed during studies by the Pennsylvania Chestnut 
Blight Commission (1913). The Commission stated that the fungus grew more rapidly on dead woody substrate than living and produced spore bearing stroma. The report also noted that dead leaves and burs produced stroma. No repetitive experiments were performed by the Commission at that time. The Commission also stated that uninfected logs that were stacked within infected zones would acquire infection and eventually sporulate. This later action was found to be more prevalent when stacked within shaded areas with elevated moisture content (Anderson and Babcock, 1913; Diller, 1965).

Chestnut is a member of the family Fagaceae. This family includes species other than chestnut that support $C$. parasitica colonization including members of the genus Quercus and Fagus. The fungus also has been reported on Rhus, Ostrya, and present but weak upon Acer, and Liriodendron (Fulton, 1912). However, classic cankers and/or debilitating symptoms do not appear unless these hosts are severely stressed or wounded (Rankin, 1914; Shear et al., 1917).

Cryphonectria parasitica is influenced by the presence of stronger and naturally occurring saprophytes. Baird (1991) demonstrated that Trichoderma spp. could grow over and potentially kill $C$. parasitica colonies in culture. Inhibition zones were formed when Cornyeum and Shaeropsis species were paired with C. parasitica in culture suggesting that they too may be antagonists. However, chi-square analysis that compared the recovery of differing C. parasitica strains with these other commonly occurring saprophytic fungi showed no significant influence upon the recovery of C. parasitica (Baird, 1991).

Other species, particularly within the family Fagaceae, have the ability to harbor C. parasitica as a parasite or saprophyte. White oak (Quercus alba L.) was reported to allow natural parasitic colonization of C. parasitica (Anderson and Babcock, 1913) and chestnut oak (Q. prinus L.) supported parasitic colonization and pycnidial formation 
when inoculated (Shear et al., 1917). Baird (1991) tested saprophytic colonization of virulent and hypovirulent $C$. parasitica upon red oak (Q. rubra L.). The virulent strain exhibited greater colonization and sporulation than the hypovirulent strain.

A previous study performed in North Carolina by Nash and Stambaugh (1982) showed that $13.8 \%$ of the scarlet oaks and $15.5 \%$ of post oak (Quercus stellata Wangenh) were infected with $C$. parasitica. Post oak has been shown to suffer severe canker symptoms and damage from the fungus (Russell et al., 1987). Torsello (1994) studied the parasitic colonization of $C$. parasitica on scarlet oak (Q. coccinea Münchh.), another member of the red oak group. They showed that $15 \%$ of the scarlet oak surveyed exhibited $C$. parasitica infected cankers. The canker types found were bole cankers $(6.3 \%)$, basal cankers $(6.9 \%)$ and both on the same tree $(1.7 \%)$. Sixty percent of the trees exhibiting cankers had evident stroma and 4.4\% had stroma without evident cankers. Perithecia were found to be present on only $3.6 \%$ of the infected specimens. They also observed that an extensive amount of $C$. parasitica "conidial ooze" was released from declining or recently dead trees. They stated that considerable amounts of inoculum were produced on the dead and dying trees in comparison with the relatively small amount of fruiting stroma found in association with cankers on living trees (Tosello, 1994).

Cryphonectria parasitica has an established reputation as a destructive parasite. However, its role as a saprophyte on American chestnut and other hosts is not well defined. Though it may not be a well-known member of the saprophytic community it is capable of colonization and sporulation on dead bark. Prospero's study indicated that pycnidia production was far greater than perithecia production on dead and dying wood (Prospero et al., 2006). This may be an important factor contributing to the spread of hypoviruses because they are disseminated only by conidia and not ascospores (Nuss, 2005). 
A pilot study conducted by King (2008), examined the effects of multiple virulent and hypovirulent strains of $C$. parasitica on living and standing dead American chestnut stems. Trees were inoculated and then girdled at various intervals. This was performed to test the sporulation and colonization rates of cankers at different times after girdling. Although a limited study, the results indicated a relation between time of girdling and increased colonization and sporulation of hypovirulent strains of the fungus. In most cases colonization and sporulation were enhanced on trees that previously had been killed by girdling before or after inoculation. Living trees used as controls showed typical canker development.

Due to the lack of an abundant American chestnut population because of its removal during blight, it is important to attempt to understand other reservoirs of inoculum. Though multiple attempts have been made, the successful use of hypovirulent strains for biocontrol has not been successful in North America (MacDonald and Fulbright, 1991). Many potential reasons exist for this situation. The inability of hypovirulent to compete with virulent inoculum in North America could be of particular importance to HV strains as biological control agents.

The following experiment is designed to expand our knowledge of the potential of C. parasitica to grow and sporulate as a saprophyte on American chestnut. Particular interest lies in the generation of inoculum by hypovirulent strains. Scarlet oak has been included in this experiment as a second host because infections of this species by $C$. parasitica commonly have been reported. When completed the experiment should provide results that compare the ability of $\mathrm{V}$ and $\mathrm{HV}$ strains to grow and produce inoculum on dead stems of both species. 


\section{METHODS and MATERIALS}

\section{$\underline{\text { Study Location }}$}

The study site was located at Bunner's Ridge (BR), Marion County, WV, near South Bunner's Ridge Road. A plantation of American chestnut was established in approximately 1985 by the WVU chestnut research group. The stand has grown without disturbance since then. In about 1998 some stems were used to test the Euro-7 hypovirulent strain for its affect on treated cankers. Some original stems remain alive and others are examples of repeated infection and death. A few infected stems that have been girdled by $C$. parasitica remain alive with healthy crowns. Fungal cultures from a few cankers on these stems yielded strains with a white HV phenotype. Extractions of dsRNA performed for this project documented that the white strains are hypovirus infected. This experiment utilizes HV and V strains from the site (Table 1).

\section{EXPERIMENTAL DESIGN AND TREATMENTS}

\section{C. parasitica Strains Selected}

Two strains of $C$. parasitica were selected for this experiment from a group of eight strains evaluated in a preliminary study (Table 1). Preliminary pathogenicity, vegetative compatibility (VC), and dsRNA extraction tests were performed to indicate the usefulness of experimental fungal strains. BR-HV-1 and BR-V-1 were recovered from the test site and met the appropriate pathogenicity, vegetative compatibility and dsRNA criteria, and were therefore used in the experiment (Figures A and B). 
TABLE 1: FUNGAL ISOLATES USED
Isolate Name
Details

Isolates used for primary experimental inoculations:

1) BR-HV-1 Isolated from Bunner's Ridge, WV (Feb, 2011)

Hypovirus content confirmed by dsRNA extraction

(March, 2011), Hypovirulent

2) BR-V-1 Isolated from Bunner's Ridge, WV (Feb, 2011), Virulent

\section{Isolates used for preliminary strain selection tests:}

3) EP-155 Isolated from Connecticut, Virulent

4) EP-146 Isolated from Pocohontas County, WV. Brown

Pigmented. Virulent

5) EP-146-HV Laboratory created transgenic (Nuss). Hypovirulent

6) Euro-7 Isolated from Florence, Italy. Hypovirulent

7) County Line Isolated from Manistee County, MI. Hypovirulent

8) Grand Haven-2 Isolated from Michigan. Virulent


Figure A: BR-V-1 (left) and BR-HV-1 (right) isolated from single spores and chosen for the experiment. 


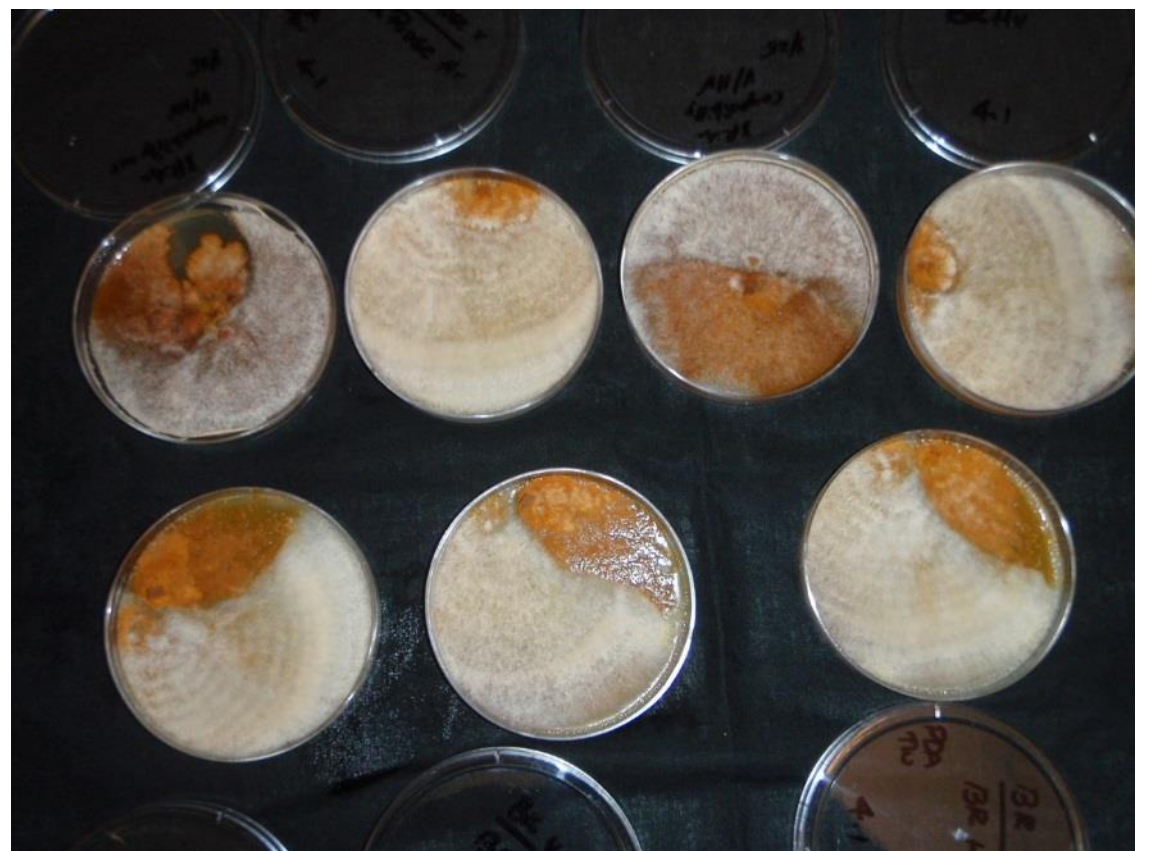

Figure B: BR-HV-1 paired with BR-V-1 to assess anastomosis frequency and Hypovirus transmission from $\mathrm{HV}$ to $\mathrm{V}$ isolate. Photo shows replicate pairings used in the experiment and hypovirus transmission to V colonies.

\section{Pathogenicity Tests}

Preliminary pathogenicity tests were conducted to determine the relative colonization rates of the Bunner's Ridge isolates in comparison to known V and HV strains (Table 1). These tests were performed on two plant tissues: Granny Smith apples and stems collected during dormancy. The ends of the stems were waxed to prevent desiccation. The chestnut stems and apples were inoculated with all isolates listed above with a $\mathrm{V}$ and $\mathrm{HV}$ isolate on the opposite linear side of each stem or apple. They were then incubated in closed, plastic bins at room temperature with a layer of vermiculite covered in aluminum foil on the bottom of the bin to aid in moisture control. Colonization and visual sporulation assessment rating (Prospero, et al., 2006) were recorded over a two-week period for the apples and after approximately 45 days for the dormant chestnut stems. Both the apples and stem inoculation sites were measured weekly to compare fungal colonization rates. 
1.) C. parasitica colonization of the stems for the entire experiment was measured using the following formula:

$$
\text { Colonization } \mathrm{cm}^{2}=\frac{\text { Length } \cdot \text { Width }}{2}
$$

This formula was used instead of the area of an elipse due to the disproportional and varied colonization patterns that became apparent throughout the course of the experiment.

\section{dsRNA Extraction}

Double-stranded RNA (dsRNA) extractions were performed on the BR-HV-1 and BR-V-1 strains to determine if they contained dsRNA, indicating hypovirus infection (Figure C). A modified dsRNA extraction procedure was used (Morris and Dodds, 1979). Known HV isolates (lanes 1-4) contain the hypovirus and a virulent isolate that does not contain the hypovirus (lane 9) were employed as controls. A black numbered lane indicates that no dsRNA was present in the isolate.



Figure C: Depicts HV and V isolates from dsRNA extraction. From left to right the isolates in each lane are: 1) GH2 2) GH2 Brown 3) Euro7 4) EP- 146 Cytoplasmic 5) Bunners Ridge HV-1 6) Bunners Ridge HV-1 (Mottled) 7) Bunners Ridge V-1 8) Bunners Ridge V-1 9) EP-155 V 


\section{Vegetative Compatibility}

Vegetative compatibility (VC) tests were periodically conducted to provide circumstantial evidence that the isolates used as inoculum were being recovered from the inoculated test stems. The VC methodology involved pairing strains on agar as described previously (Anagnostakis, 1977), with the amendment of brome-cresol green to the medium (Powell, 1995) (Appendix Table 16).

\section{Stem Origination, Labeling and Selection}

American chestnut stems used in this study were cut from the BR site and the scarlet oak stems were cut from the BR site and the WVU forest. Stems were assigned numbers in the order of cutting so that the origin of the stem position on the tree was known. They were then cut into $\sim 60 \mathrm{~cm}$ pieces and the cut ends labeled with letters in sequential order from the bottom to the top of the tree. The stems were divided randomly among five replicate groups. The group name where the treatment was located and species also were included. For example, a stem labeled, G1-1a-chestnut, G1-1bchestnut, etc., indicated two successive stems from the same tree within group 1. Stem diameters were measured before placement within stacks.

The requirement for these stems to be selected was at least 60 -cm length of healthy bark free from infection or wounds. Due to the widespread chestnut blight disease within the stand, chestnut stems were limited in size and age in comparison to oak. The chestnut stem diameters ranged from 2.8 to $7.5-\mathrm{cm}$ on young saplings growing without a shading overhead canopy. Therefore, the chestnut bark was typically not very thick or furled. In contrast, the scarlet oak was difficult to find in the young sapling stage and when small trees were located they tended to be older due to stunted shade tolerant growth under canopy or upon the forest edge with partial shade. Oak saplings of similar 
size, age and bark thickness to chestnut were not readily available and some upper level tree branches and main stems had to be utilized. Therefore, oak stems had greater variation in stem size ranging from 3.1 to $9.5-\mathrm{cm}$ in diameter. The oak bark also tended to be thicker and more furled (Figure 1).

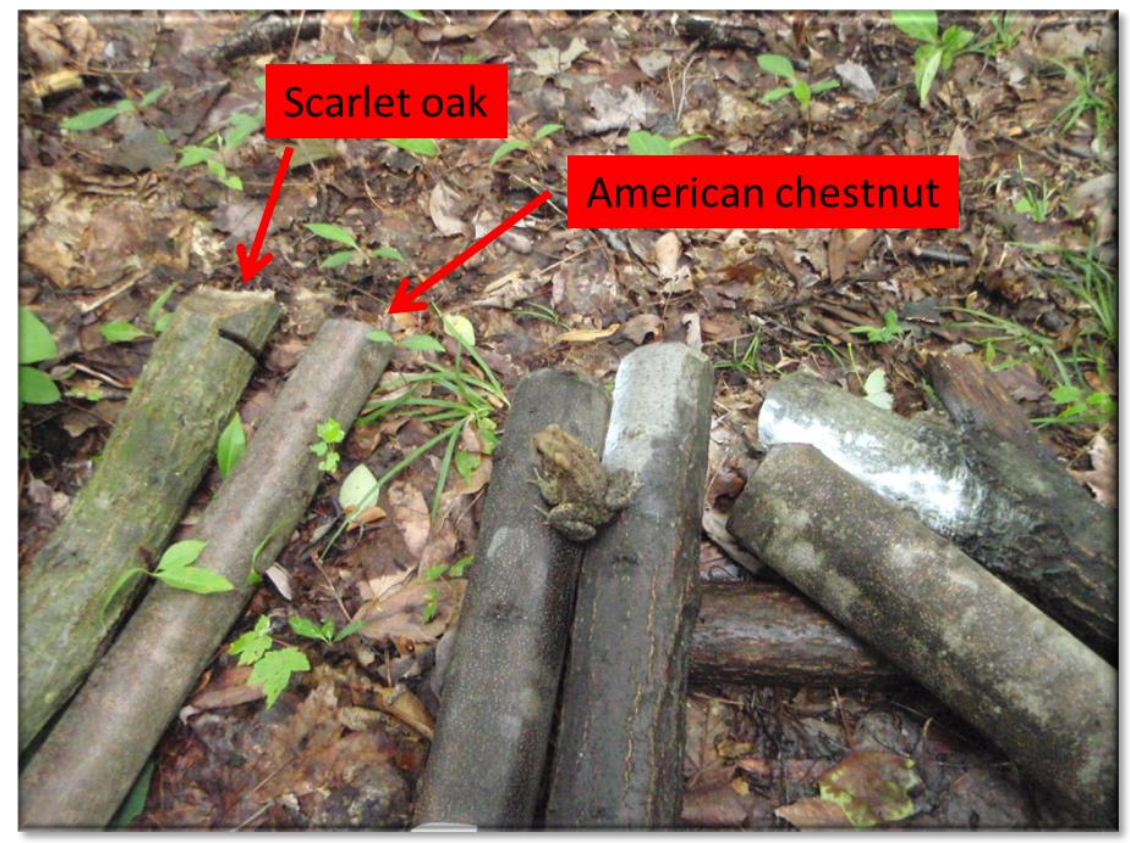

Figure 1: Photograph of typical American chestnut and scarlet oak stems used for this experiment side-byside. Bufo americanus appeared by chance and is not subject to inference.

\section{$\underline{\text { Stacks }}$}

The experiment was established at the edge of the forest under the canopy to the northeast of the chestnut plantation. The stems of American chestnut and scarlet oak were cut the week immediately preceding the creation of the stacks. Fifteen stacks of stem pieces were constructed from the healthy stems (Figure 2). They were divided into five groups each consisting of three stacks per group with twenty-four stems per stack arranged in four layers. A total of eighteen test specimens were placed in each stack with a bottom layer composed of six sourwood (Oxydendrum arboreum (L. DC.) stems that act as a buffer between the ground and the test stems. Each stack was constructed in a triangular fashion with three layers of six stems per level to be inoculated (Figure 2 and Appendix Figure 59). An equal number of American chestnut and scarlet oak stems were 
arranged side-by-side in each layer. The bottom layer of the stack closest to the ground was designated as Row-1, Row-2 was in the middle and Row-3 was on top. The groups of three stacks were labeled G-1 to G-5 with each stack of stems designated to receive a different inoculum type of either BR-V-1, BR-HV-1 or water agar control (Table 1). Each group contains one of each inoculum type resulting in five replicate stacks, with 90 total stems per treatment (Figure 2). Pin flags were utilized to indicate the treatment type and group number.

Densiometer readings for shading were recorded under full leaf condition of the canopy trees. The densitometer readings were (where $0=$ full shade; and $100=$ full open sun) $\mathrm{G}-1=5, \mathrm{G}-2=1, \mathrm{G}-3=3, \mathrm{G}-4=2$, and $\mathrm{G}-5=1$. The densitometer readings therefore indicate that shading was very high at $>95 \%$ at all group locations.



Figure 2: Six out of the fifteen stacks in two out of the five groups comprised of American chestnut and scarlet oak inoculated with V, HV or control inoculum.

\section{Inoculum Production and Inoculation Procedure}

There were three inoculation periods. The first was on May $20^{\text {th }}, 2011$ and subsequent inoculations were on August $4^{\text {th }}$ and October $4^{\text {th }}$. A slurry inoculum was made of $250 \mathrm{~mL}$ of $0.1 \%$ peptone water, $250 \mathrm{~mL}$ of $2.5 \%$ solidified water agar, and ten (10-day-old) potato dextrose agar (PDA; 10-cm diameter culture containing 
approximately $30 \mathrm{ml}$ of medium) (Appendix Table 16) cultures of BR-V-1 and BR-

HV-1 inoculum types. The water agar slurry contained no inoculum. All components were then placed in a 4-L Waring blender and mixed together. The slurry then was poured into a $500 \mathrm{~mL}$ plastic squeeze bottle to dispense for the inoculations. Inoculation slurry was applied to stems within 24 hours of preparation. BR-V-1, BR-HV-1 and water agar control isolates were assigned to each stack within a group. A randomly selected layer of stem pieces in each stack was inoculated during each period. Inoculation wounds were created using a hammer and $1-\mathrm{cm}$ diameter steel leather punch, penetrating to the wood just below the cambium. Inoculum was applied to the punched stem holes. The inoculum filled holes were covered with time tape for protection until the fungus was considered established within host substrate. Establishment was considered adequate, at the time of the first measurement one month later, following the inoculation.

\section{Timeline for canker colonization measurements and sporulation evaluation}

All treatment types were monitored and evaluated for changes in colonization, stroma formation (sporulation) and appearance. The inoculation sites were examined at monthly intervals following the initial inoculation. Colonization was measured from the point of inoculation or until the fungus either completely girdled and/or reached the end

of the stem. Sporulation also was evaluated by subjectively scoring the number of stroma associated with each infection. A zero-to-three scale was used (Prospero et al., 2006) (Table 1a). Data were collected through the winter of 2011.

\section{Sampling Procedure}

Two sizes of bark samples were collected from the inoculated stems during each measurement period. A bone biopsy instrument and a steel leather punch were used 
resulting in 2-mm plugs and 10-mm diameter disc samples, respectively. The 2-mm plugs were used for culturing resident fungi and the $10-\mathrm{mm}$ discs were used for examining individual pycnidia and conducting single spore analysis because intact stroma were recoverable from the larger surface area provided by the $10-\mathrm{mm}$ diameter disc.

Eight 2-mm plugs were collected from each artificially initiated canker using the bone biopsy instrument, six from within the perceived colonized region and two from approximately two centimeters beyond the boundary of visible stoma (Figure 3). The subsequent two samples taken from outside the perceived colonized region were cultured for presence or absence of $C$. parasitica. This was performed to assess if the actual colonization went beyond the perceived colonized area on the stem to prove the colonization limits by $C$. parasitica. The plug samples were then placed within labeled 96-well microtiter plates with the two external to apparent colonized region samples placed on the far end. This allowed reference to the sample site on the stem. The samples within the plates were kept frozen for preservation prior to processing. Samples were cultured to determine if the strain recovered is the same as that used to initiate the infection and for assessment of other resident fungi within the canker. The resulting cultures were scored by morphology to determine whether they were V or HV strains of C. parasitica or other microorganisms.

The 10-mm bark discs containing stroma also were collected by hammering a steel leather punch into the bark to the cambium and extracting the plug. The samples were stored in labeled 24-well culture plates and kept frozen prior to processing. The plugs provided fruiting structures of $C$. parasitica or other microorganisms for analysis. Dissecting and compound microscopes were used to examine the fruiting bodies on the 10-mm discs and cultures created from the 2-mm plugs. When pycnidia were identified 
the spores they produced were subjected to single spore analysis to assess whether they would yield V or HV inoculum.

Stem colonization measurements were made from the inoculation site out by length and width. Appearance of stroma and changes in bark appearance and color were used as direct indicators of fungal colonization. When stroma were not directly evident, orange coloration under the periderm of the bark (Figure 4) and a sunken appearance typical of cankers on living trees infected with C. parasitica were indicators of the colonization limits. The boundaries between colonized and non-colonized bark were confirmed by sampling within the perceived infection and $2-\mathrm{cm}$ external to the lengthwise infection boundary (Figure 3).

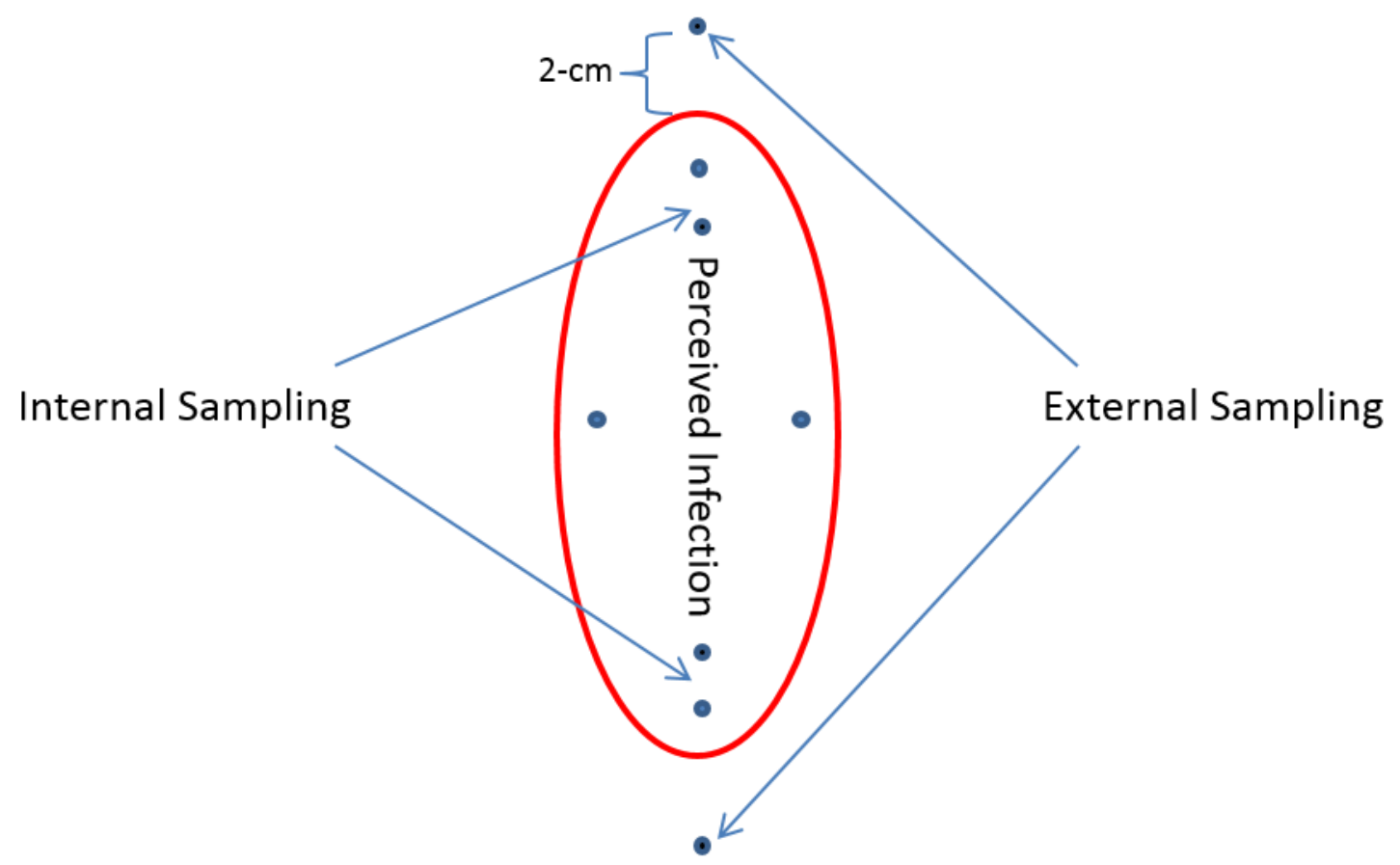

Figure 3: Example of bone marrow biopsy instrument sampling design. This method was used to assess whether sampling was accurately depicting the infection limits on the inoculated stem. 


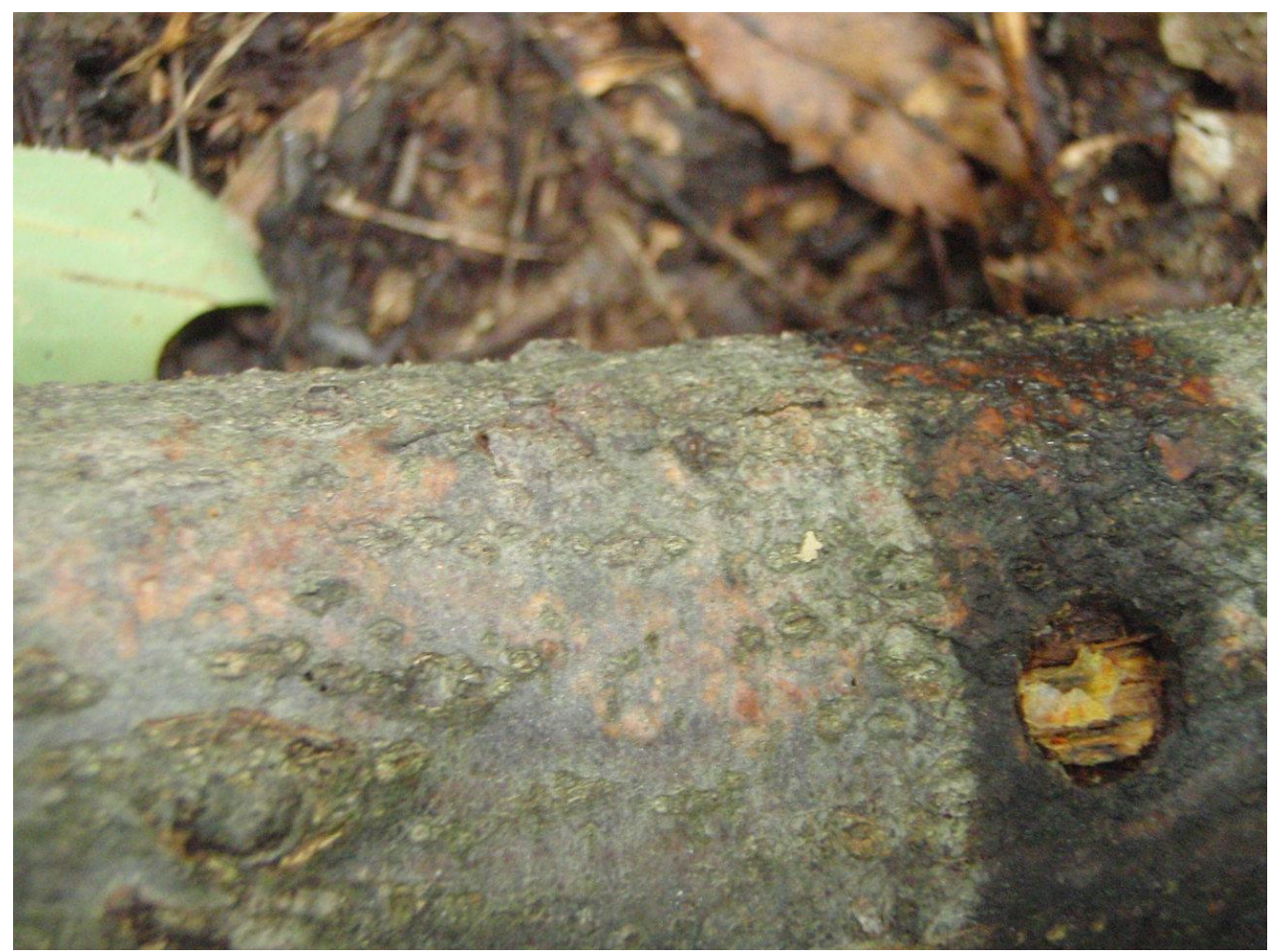

Figure 4: Showing orange discoloration present in bark used as a C. parasitica infection indicator when stroma were not present. Sampling methods confirmed infection.

\section{$\underline{\text { Culture and Single Spore Procedures }}$}

The 2-mm bark plugs were sterilized in $10 \%$ bleach solution before culture. After five-days incubation on glucose-yeast extract agar (GYE/A), the resulting fungi were transferred to potato dextrose agar (PDA) (Appendix Table 16) and incubated at $20^{\circ} \mathrm{C}$ for ten days in a 16:8 hour light regime. The resulting fungal isolates were identified as $C$. parasitica or other fungi. If the isolates were C. parasitica, culture morphology was used to assess whether the isolates were $\mathrm{V}$ or $\mathrm{HV}$.

The 10-mm bark discs were used to collect spores from pycnidia. Single pycnidia were isolated from each bark disc and added to $1 \%$ peptone solution in a watch glass. Spores recovered from pycnidia were serially diluted and plated on GYE and spread over the surface of the agar with a glass L-shaped rod. Spores were then incubated at $30^{\circ} \mathrm{C}$ in total darkness for 48 hours. Germinating conidia were then identified and transferred to 
PDA, 5 per plate. The germlings that resulted were placed in the $20^{\circ} \mathrm{C}$ room for 5 days and frequency of $\mathrm{V}$ and $\mathrm{HV}$ colonies were evaluated based on culture morphology.

\section{Fungi other than $C$. parasitica}

Commonly occurring fungi other than $C$. parasitica were identified by isolation and subsequent culture. Samples were taken for isolates at monthly intervals during each measurement period. Individual cumulative percentages were then tallied separately for each inoculation period. Identification to genus and when possible species level was confirmed through traditional morphological keys (Barnett and Hunter, 1998) and/or a polymerase chain reaction (PCR) procedure. Universal fungal primers targeting the internal transcribed space regions of ribosomal DNA for ITS-1 and ITS-4 were used for the amplification process and molecular confirmation (White et al., 1990). The following primer sequence was used:

Primer ITS1 - 5'TCCGTAGGTGAACCTGCGG3' Primer ITS4-5'TCCTCCGCTTATTGATATGC3'

PCR products were then shipped to Davis Sequencing (Davis, CA) to obtain the DNA sequence. The results from Davis Sequencing were then analyzed and compared for the best match within the National Center for Biotechnology Information (NCBI) BLAST database (http://blast.ncbi.nlm.nih.gov/Blast.cgi) (Altschul, et al., 1997). A record of the frequency of other species associated with the stems was kept and tallied as a percentage relative to $C$. parasitica cultures recovered from the same infection.

\section{$\underline{\text { Dissemination of HV strains }}$}

Dissemination of the strains used for inoculation to non-inoculated stems within stacks also was assessed. To accomplish this, one healthy stem of American chestnut and 
scarlet oak was hammered vertically into the ground within the middle of each stack to serve as a trap stem (Figure 4a1). These stems were sampled when stromata of $C$. parasitica were evident. Isolates recovered from these stems were evaluated to determine whether dissemination of saprophytically produced $\mathrm{HV}$, or $\mathrm{V}$ inoculum to these stems has occurred. Isolates from these stems also were subject to single spore analysis and vegetative compatibility testing. These tests circumstantially determined if these putative $\mathrm{HV}$ isolates were disseminated from the original test stems based on morphology, pigmentation, spore abundance per pycnidium, hypovirus transmission rates and $\mathrm{VC}$ in comparison to preliminary tests.



Figure 4a1: Represents an experimental stack with two stems, one oak and one chestnut, hammered into the ground in the stack center. These stems were un-inoculated and intended to show if V and HV C. parasitica from within the stack would infect these stems.

\section{Sporulation Ranking}

Sporulation was ranked using a four tier ordinal rating system ranging from zero (0) to three (3) where zero represents no sporulation and three represents extensive sporulation (Prospero et al., 2006). The ranking inspection is subjective in terms of observing the 
infection site and making a visual judgment. The judgment is based upon the total number of stroma and the distance between them as well as the distance of spread from the original inoculation site (Figures $4 \mathrm{a}-4 \mathrm{~h}$ ).

Table 1a: Ordinal rating system used to assess sporulation (Prospero et al., 2006)

2.) $0=$ no evidence of sporulation

3.) $1=$ low sporulation

4.) $2=$ medium sporulation

5.) 3 = heavy sporulation



Figure 4a: Showing limited stroma $=$ Spore Rank 1 


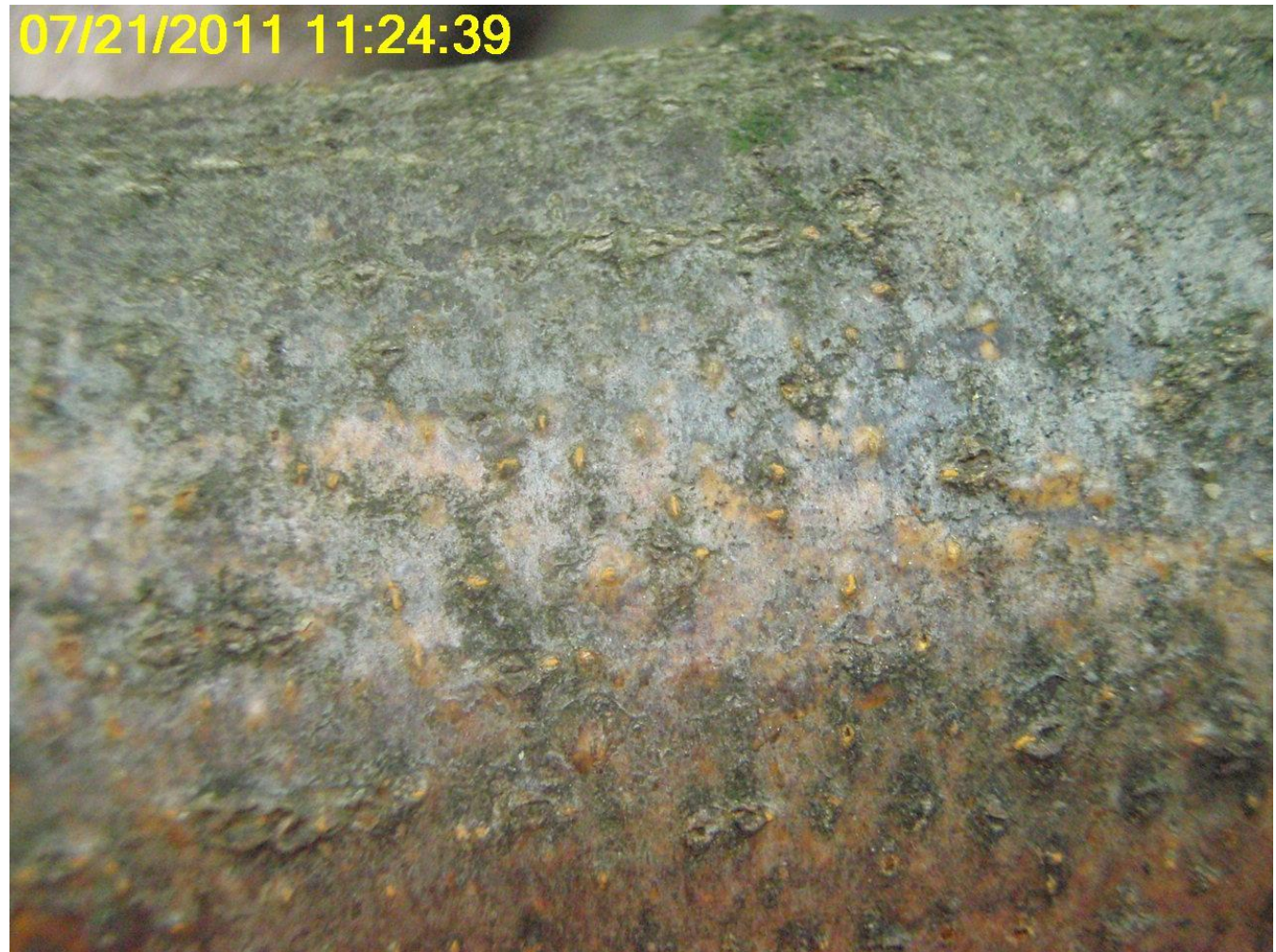

Figure 4b: Showing maximum limited stroma $=$ Spore Rank 1



Figure 4c: Showing limited stroma $=$ Spore Rank 2 


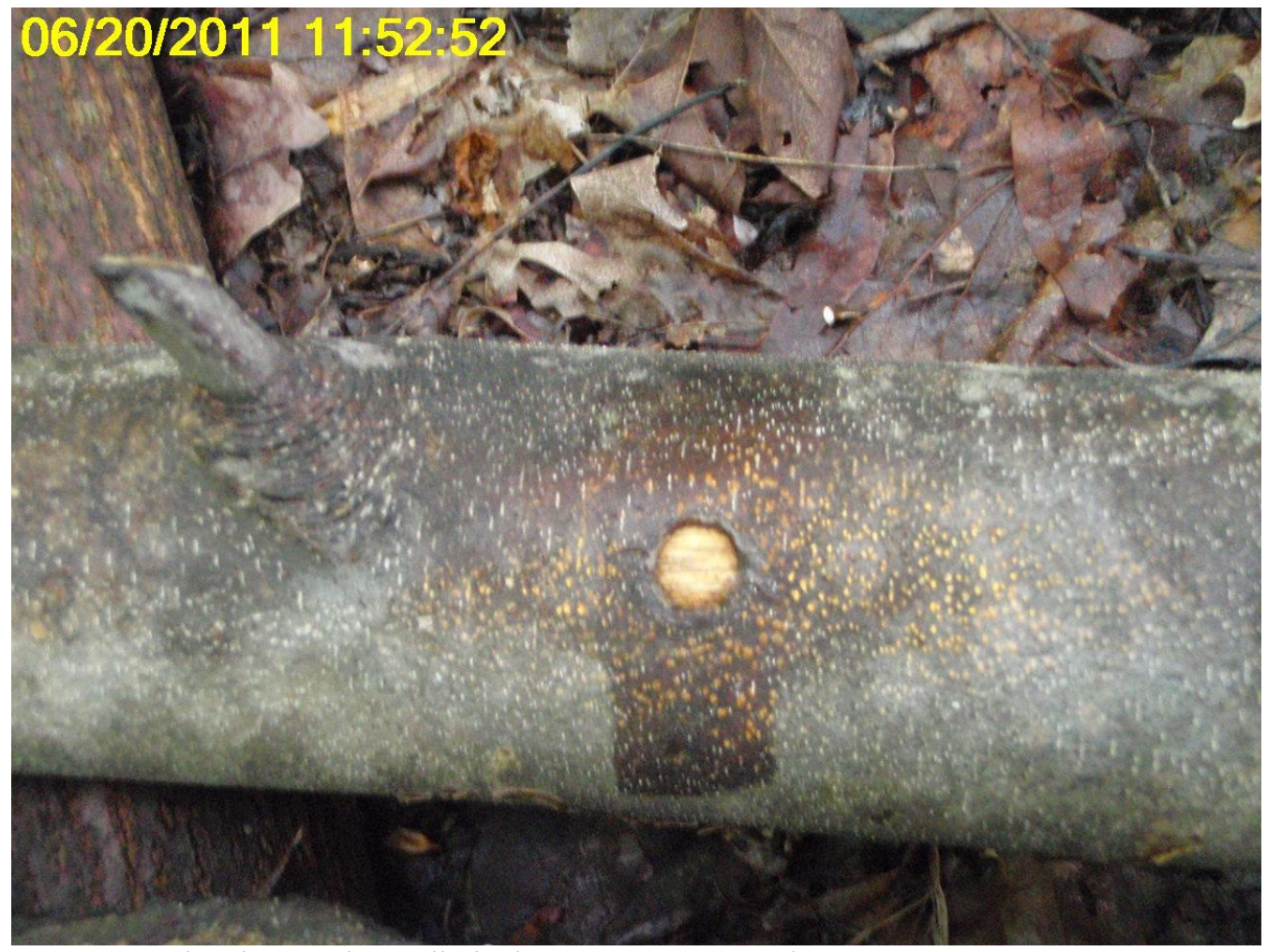

Figure 4d: Showing maximum limited stroma $=$ Spore Rank 2

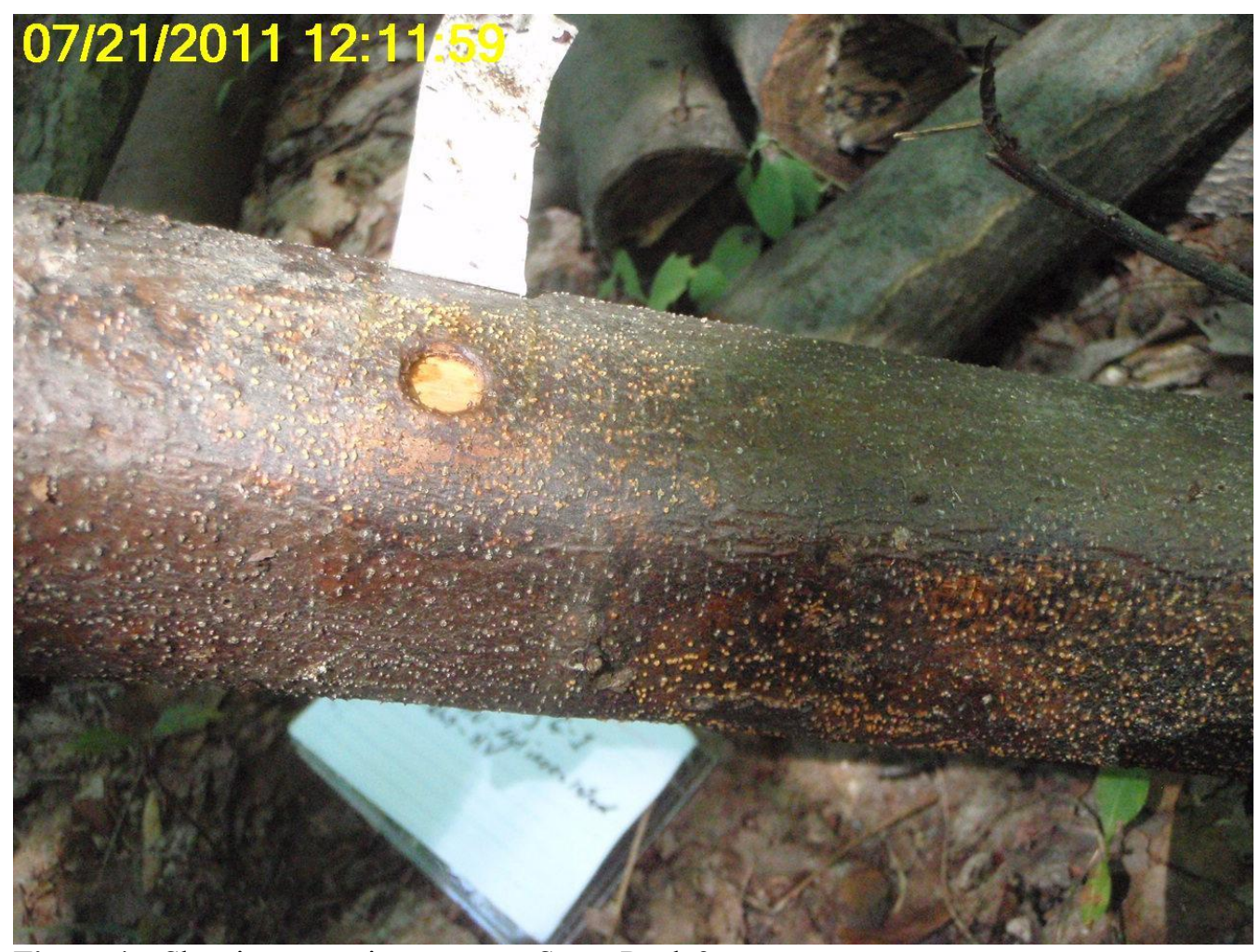

Figure 4e: Showing extensive stroma $=$ Spore Rank 3 


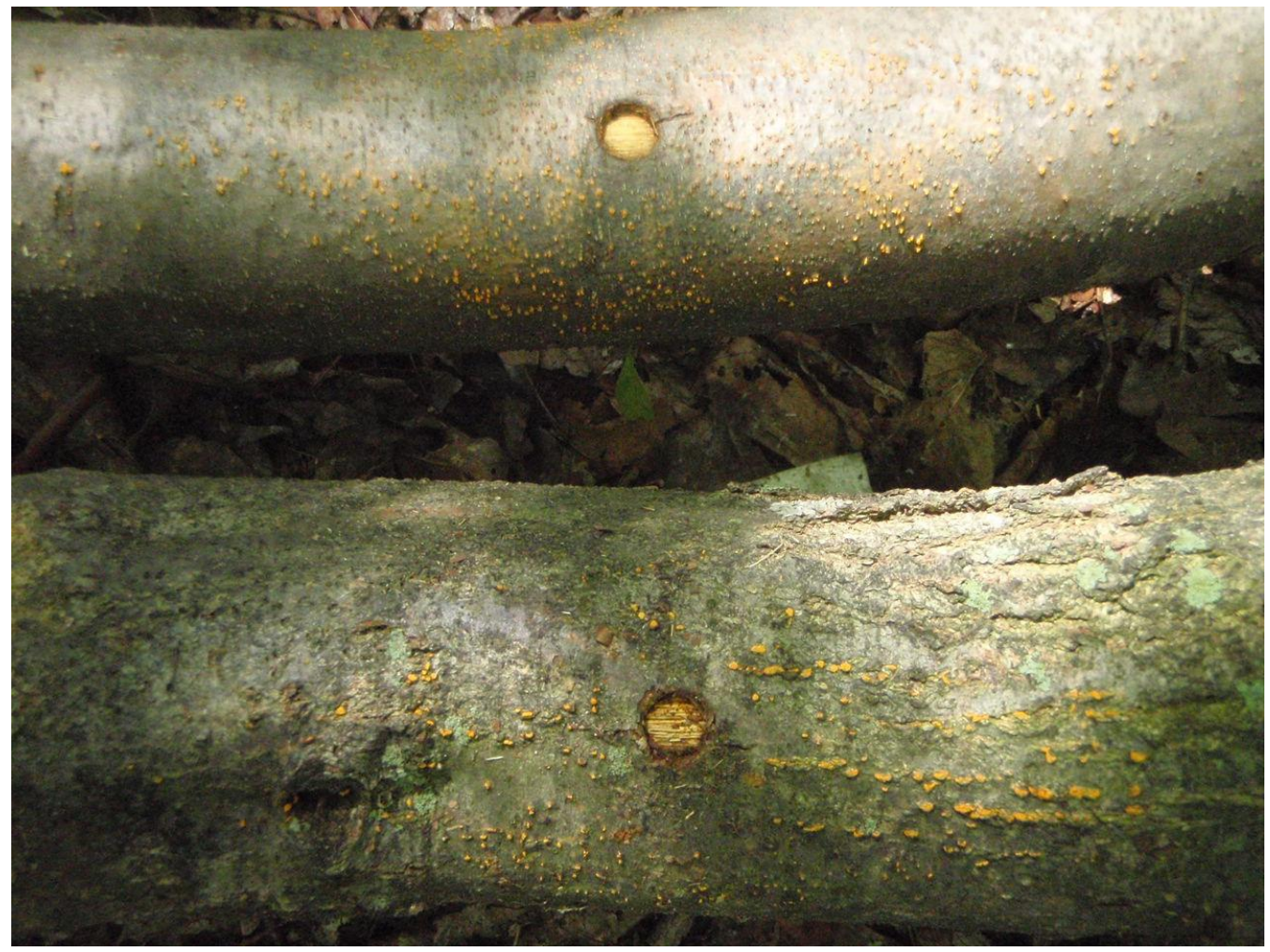

Figure 4f: Showing extensive stroma at top stem $=$ Spore Rank 3

Lower stem showing maximum limited stroma $=$ Spore Rank 2

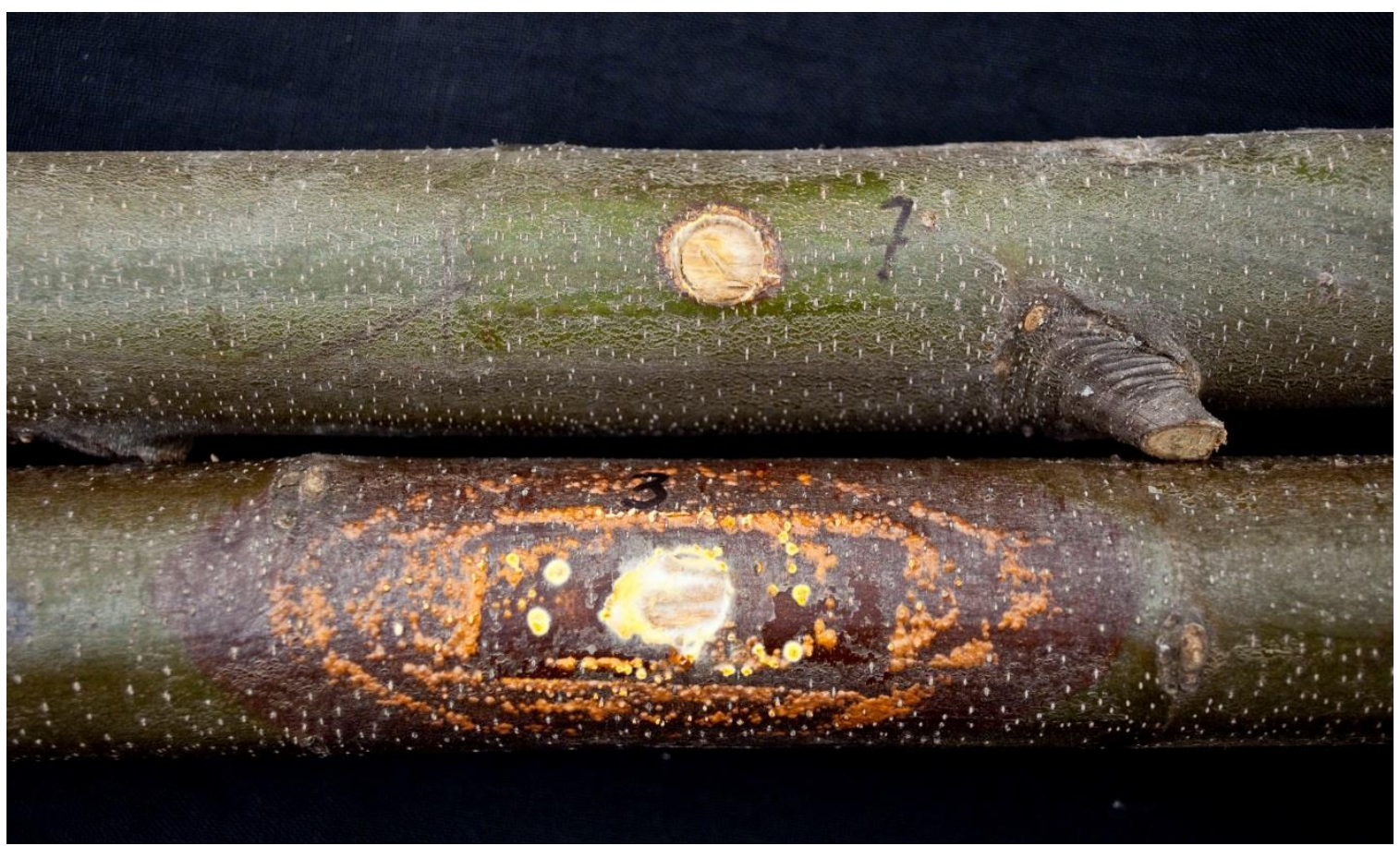

Figure 4g: Showing minimum stroma at top stem $=$ Spore Rank 1

Lower stem showing extensive stroma $=$ Spore Rank 3 


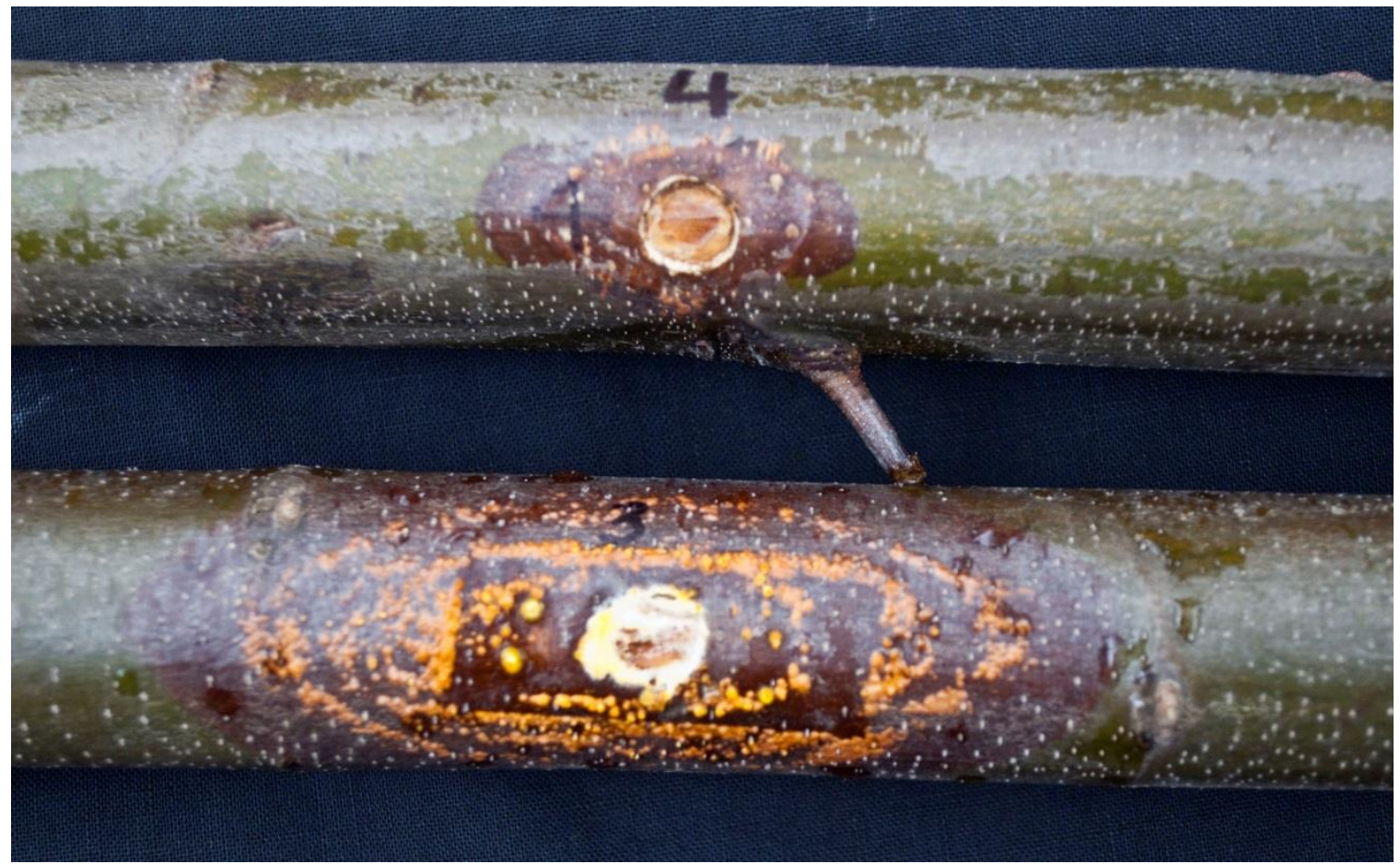

Figure 4h: Showing minimum stroma at top stem $=$ Spore Rank 2

Lower stem showing extensive stroma $=$ Spore Rank 3

\section{$\underline{\text { Statistics }}$}

The programs JMP and Microsoft Excel were used to perform the statistics and graphs on all data. An alpha $(\alpha=0.05)$ was used throughout the experiment. A Bonferroni correction was performed on the set alpha whenever appropriate during multiple comparisons. Standard T-Tests and one way ANOVA's were utilized for comparisons of significant evidence in the mean data to suggest differences. A Tukey-Kramer multiple comparisons test was then used to determine which specific differences occurred when variances were equal. When variances were not equal a LOG10(+1) conversion was performed in attempt to normalize the data set. In cases where data variance was not successfully normalized Steel-Dwass all pairs and Wilcoxon non-parametric tests were utilized. Variances were analyzed before any other tests were performed using the Brown-Forsyth and Leven's tests for unequal variances. 


\section{RESULTS}

\section{CHAPTER 1: COLONIZATION}

Three inoculation periods (IP's) were used to assess colonization of $C$. parasitica on American chestnut and scarlet oak stems. Measurement of the bark tissue colonized was used as a variable to examine the differences in $\mathrm{V}$ and $\mathrm{HV}$ colonization of the inoculated stems. The first analysis compared the colonization of V and HV for the two tree species. A second analysis considered whether position (layer) in the stack had an effect on colonization and the third evaluated colonization differences among groups due to their placement at the research site. Measurements of colonization were pooled from the first to the last time period to assess total colonization over the entire period of the experiment and analyzed by a one way ANOVA using $\alpha=0.05$.

\section{SECTION 1: Analysis of Total Colonization}

\section{First Inoculation Period}

Even though V colonization was greater on oak than on chestnut (Figure 5) results indicated there was not sufficient evidence that American chestnut was different than scarlet oak for the May inoculation period $(\mathrm{P}>\mathrm{F}=0.4909)$ (Figure 5). The exception was, at the time of the measurement period on June $20^{\text {th }}$, the virulent fungus had grown significantly more on chestnut than on oak $(\mathrm{P}>\mathrm{F}=<0.0001)$. Thereafter, measurements indicated more colonization on oak than chestnut but the remainder of the monthly measurements were not significantly different.

When HV mean colonization was evaluated, American chestnut was not significantly different than scarlet oak for the May inoculation $(\mathrm{P}>\mathrm{F}=0.4729)$ (Figure 5). Though, the HV colonization was significantly better on chestnut for the first measurement taken on June $20^{\text {th }}(\mathrm{P}>\mathrm{F}=<0.0001)$, colonization differences were non- 
significant at $\alpha=0.05$ for the remaining five measurement periods. Similar to the $\mathrm{V}$ fungus, $\mathrm{HV}$ eventually grew better on oak throughout this inoculation period than on chestnut and by the end of the test, had higher average colonization than chestnut for both $\mathrm{V}$ and HV inoculum types (Figures 5 and 6). The total summed cumulative colonization for each sampling period over the duration of the experiment is illustrated in Figure 6. These data were summed separately for each individual IP and treatment type to show the collective colonization over time by $C$. parasitica of all stems for each measurement period. Therefore, the sum of cumulative colonization exceeds the area of an individual stem. This applies to all inoculation periods.

When colonization between $\mathrm{V}$ and $\mathrm{HV}$ was compared, the V isolate grew significantly better on both American chestnut and scarlet oak $(\mathrm{P}>\mathrm{F}=<0.0001)$ (Figure 6). Overall, V and HV had the greatest average colonization on scarlet oak. The water agar inoculated control stems showed almost no colonization by $C$. parasitica compared to the inoculated stems $(\mathrm{P}>\mathrm{F}=<0.0001)$ and had essentially null colonization at the established inoculation points (Figure 6). Some infections occurred at the cut ends of the stems but were not considered in the experiment. 




Figure 5: Average colonization for V BRV-1 and HV BRHV-1 not significantly different between scarlet oak and American chestnut at $\alpha=0.05(\mathrm{P}>\mathrm{F}=0.4909)$ and $(\mathrm{P}>\mathrm{F}=0.4729)$ respectively, for the first inoculation period from May $20^{\text {th }}$ to December $8^{\text {th }} 2011$.

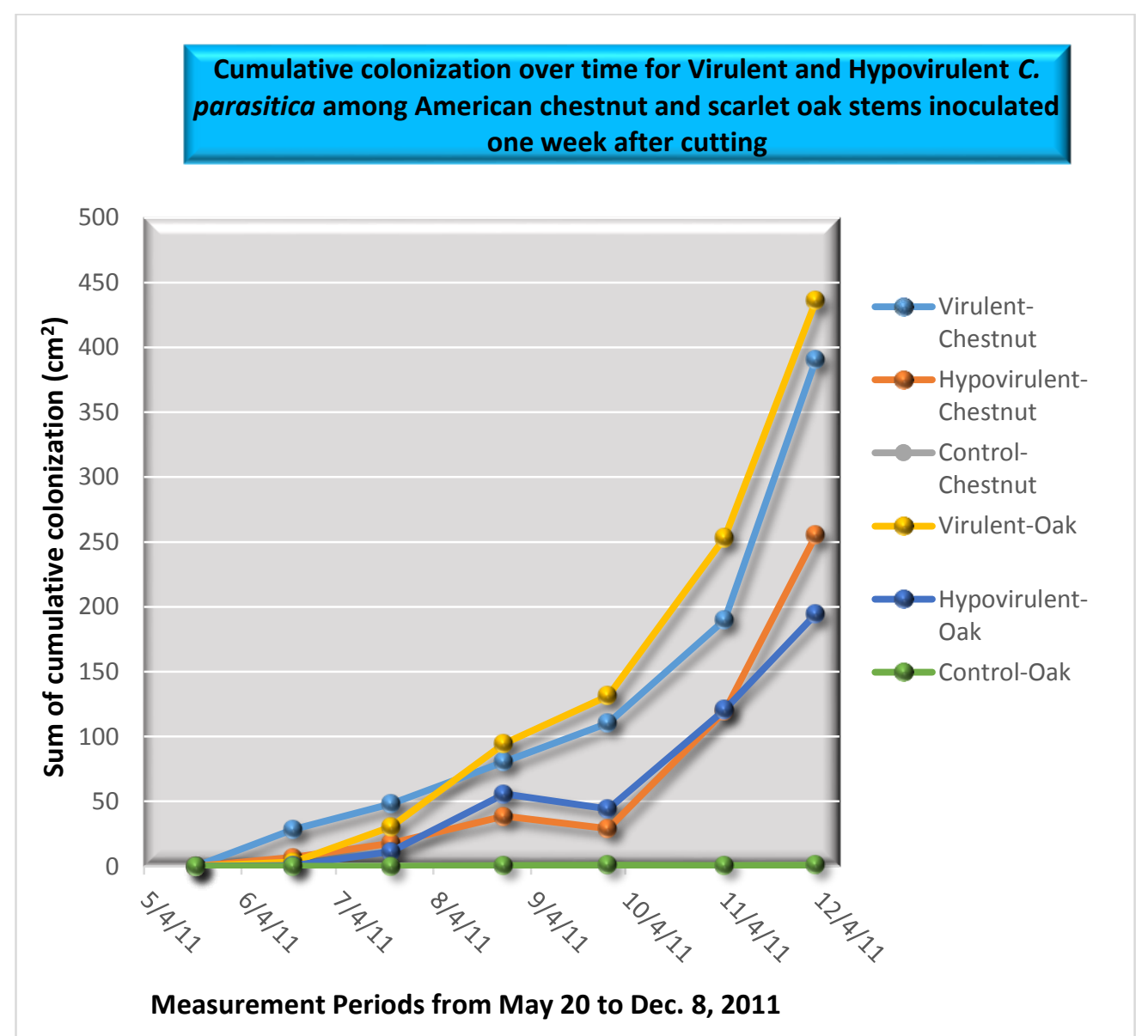

Figure 6: Sum of cumulative colonization for V BRV-1 and HV BRHV-1 were significantly different at $\alpha=0.05(\mathrm{P}>\mathrm{F}=0.0001)$ when $\mathrm{V}$ and $\mathrm{HV}$ was compared on American chestnut and the same for scarlet oak for the first inoculation period from May $20^{\text {th }}$ to December $8^{\text {th }} 2011$. When Control stems were compared with $\mathrm{V}$ and $\mathrm{HV}$ inoculations, the Control expressed nearly null colonization at the inoculation sites $(\mathrm{P}>\mathrm{F}=$ $0.0001)$. 
$\underline{\text { Second Inoculation Period }}$

The second inoculation period was initiated on August $4^{\text {th }}, 2011$ and measured the same colonization factors as the first period. Like the first inoculation period there was not sufficient evidence that the mean difference of V C. parasitica colonization on American chestnut was different than scarlet oak $(\mathrm{P}>\mathrm{F}=0.0770)$. Even though colonization was better on oak than on chestnut, the means were not significantly different (Figure 7). Colonization by the $\mathrm{V}$ isolate also was greater but not significantly more by the time of the first measurement on September $7^{\text {th }}(\mathrm{P}>\mathrm{F}=<0.0666)$ wherein the fungus grew more on oak than chestnut. The remainder of the monthly colonization measurements did approach significance.

The mean colonization of HV C. parasitica on American chestnut was significantly less than scarlet oak for the August $4^{\text {th }}$ inoculation $(\mathrm{P}>\mathrm{F}=0.0349)$ (Figure 7). For $\mathrm{V}$ and $\mathrm{HV}$ inoculum the fungus grew better on oak than chestnut. The cumulative colonization for each sampling period over the duration of the experiment is illustrated in Figure 8.

When total colonization of V to HV were compared, V colonization was not significantly greater than $\mathrm{HV}$ colonization for either American chestnut $(\mathrm{P}>\mathrm{F}=<0.4666)$ or scarlet oak $(\mathrm{P}>\mathrm{F}=<0.4615)$ (Figure 8). Overall, $\mathrm{V}$ and $\mathrm{HV}$ had the greatest average colonization on scarlet oak. The water agar inoculated control stems essentially showed no colonization when compared to the inoculated stems $(\mathrm{P}>\mathrm{F}=<0.0001)$ and were essentially null at the established inoculation points (Figure 8). End infections occurred at the cut ends of the stems but were not considered in the experiment. 




Figure 7: Average colonization for V BRV-1 and HV BRHV-1 not significantly different for $\mathrm{V}$ but was on HV between scarlet oak and American chestnut at $\alpha=0.05(\mathrm{P}>\mathrm{F}=0.0770)$ and $(\mathrm{P}>\mathrm{F}=0.0349)$ respectively, for the second inoculation period from August $4^{\text {th }}$ to December $8^{\text {th }} 2011$.

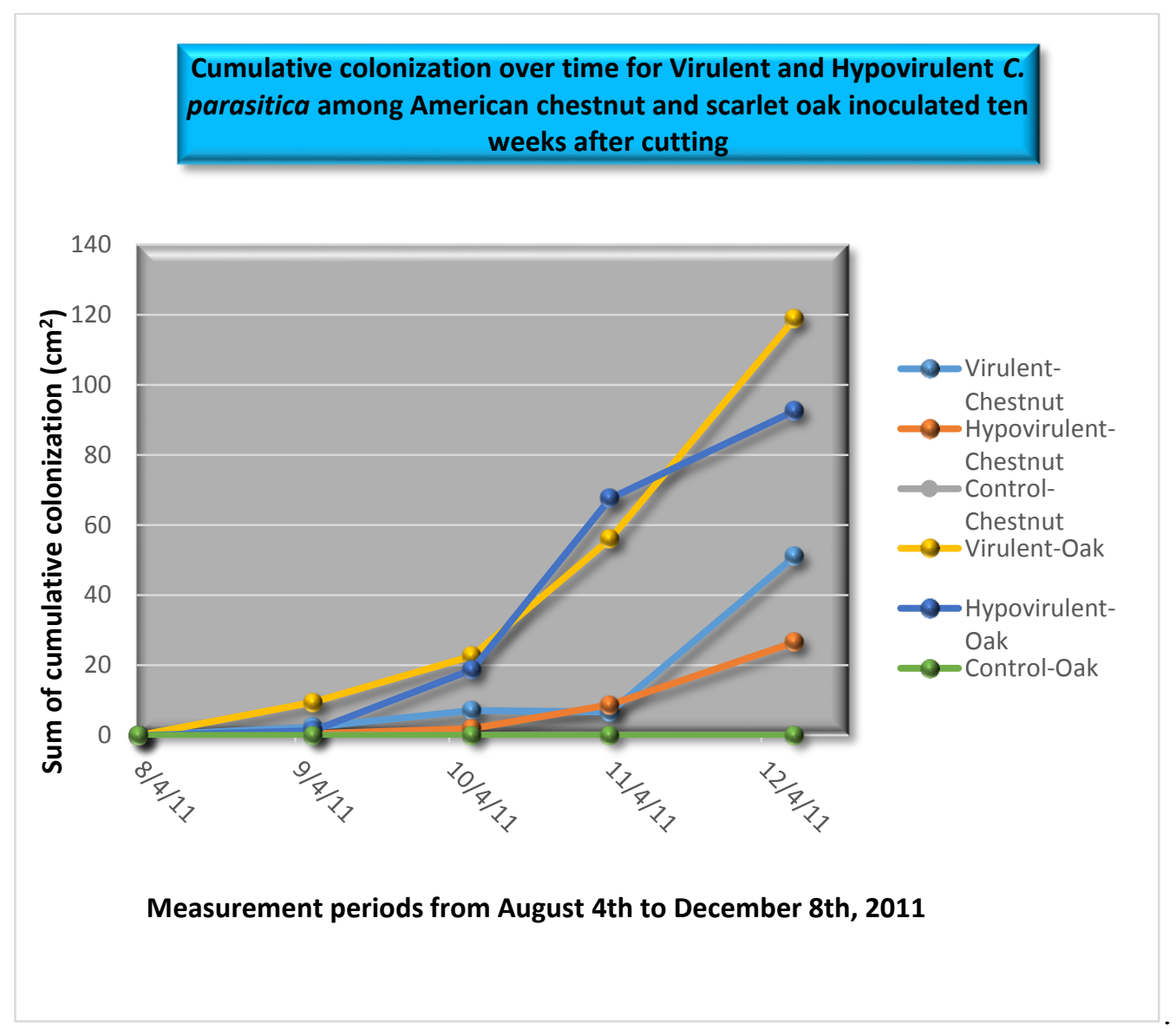

Figure 8: Sum of cumulative colonization for V BRV-1 and HV BRHV-1 were not significantly different at $\alpha=0.05$ when $\mathrm{V}$ and HV was compared on American chestnut $(\mathrm{P}>\mathrm{F}=<0.4666)$ and the same for scarlet oak $(\mathrm{P}>\mathrm{F}=<0.4615)$ for the second inoculation period from August $4^{\text {th }}$ to December $8^{\text {th }} 2011$. When Control stems were compared with $\mathrm{V}$ and HV inoculations, the Control expressed nearly null colonization at the inoculation sites $(\mathrm{P}>\mathrm{F}=0.0001)$. 


\section{Third Inoculation Period}

The third inoculation period was initiated on October $4^{\text {th }}, 2011$. For this period there was not sufficient evidence that the mean difference of $\mathrm{V} C$. parasitica colonization on American chestnut was different than scarlet oak $(\mathrm{P}>\mathrm{F}=0.1919)$. Even though colonization, was much greater on oak than on chestnut it was not significantly so (Figure 9). Virulent fungal colonization also was greater but not significantly so on November $7^{\text {th }}(\mathrm{P}>\mathrm{F}=<0.1642)$ at which time the fungus had grown more on oak than chestnut. The remainder of the monthly measurements also had greater colonization on oak but not significantly so.

The mean colonization of HV C. parasitica on American chestnut was not significantly less than scarlet oak for the October $4^{\text {th }}$ inoculation $(\mathrm{P}>\mathrm{F}=0.30910)$ (Figure 9). For $\mathrm{V}$ and $\mathrm{HV}$ inoculum the fungus grew better on oak than chestnut. The cumulative colonization for each sampling period over the duration of the experiment is illustrated in Figure 10.

When V to HV total colonization were compared, V colonization was not significantly greater than $\mathrm{HV}$ colonization for either American chestnut $(\mathrm{P}>\mathrm{F}=<0.3778)$ or scarlet oak $(\mathrm{P}>\mathrm{F}=<0.4255)$ (Figure 10). Overall, $\mathrm{V}$ and $\mathrm{HV}$ had the greatest colonization on scarlet oak. The water agar inoculated control stems essentially showed no colonization when compared to the inoculated stems $(\mathrm{P}>\mathrm{F}=<0.0001)$ and were essentially null at the established inoculation points (Figure 10). End infections occurred at the cut ends of the stems but were not considered in the experiment. 


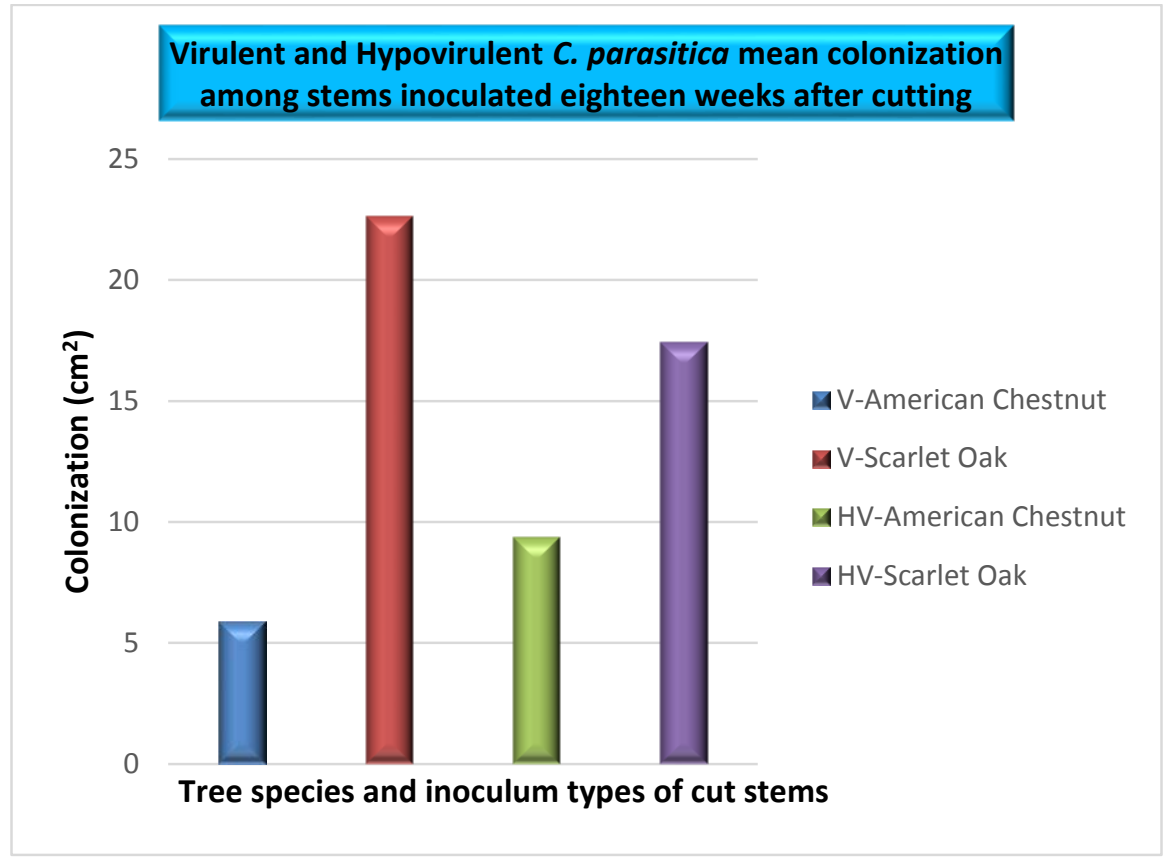

Figure 9: Average colonization for V BRV-1 and HV BRHV-1 not significantly different between scarlet oak and American chestnut at $\alpha=0.05(\mathrm{P}>\mathrm{F}=0.1919)$ and $(\mathrm{P}>\mathrm{F}=0.30910)$ respectively, for the second inoculation period from October $4^{\text {th }}$ to December $8^{\text {th }} 2011$.

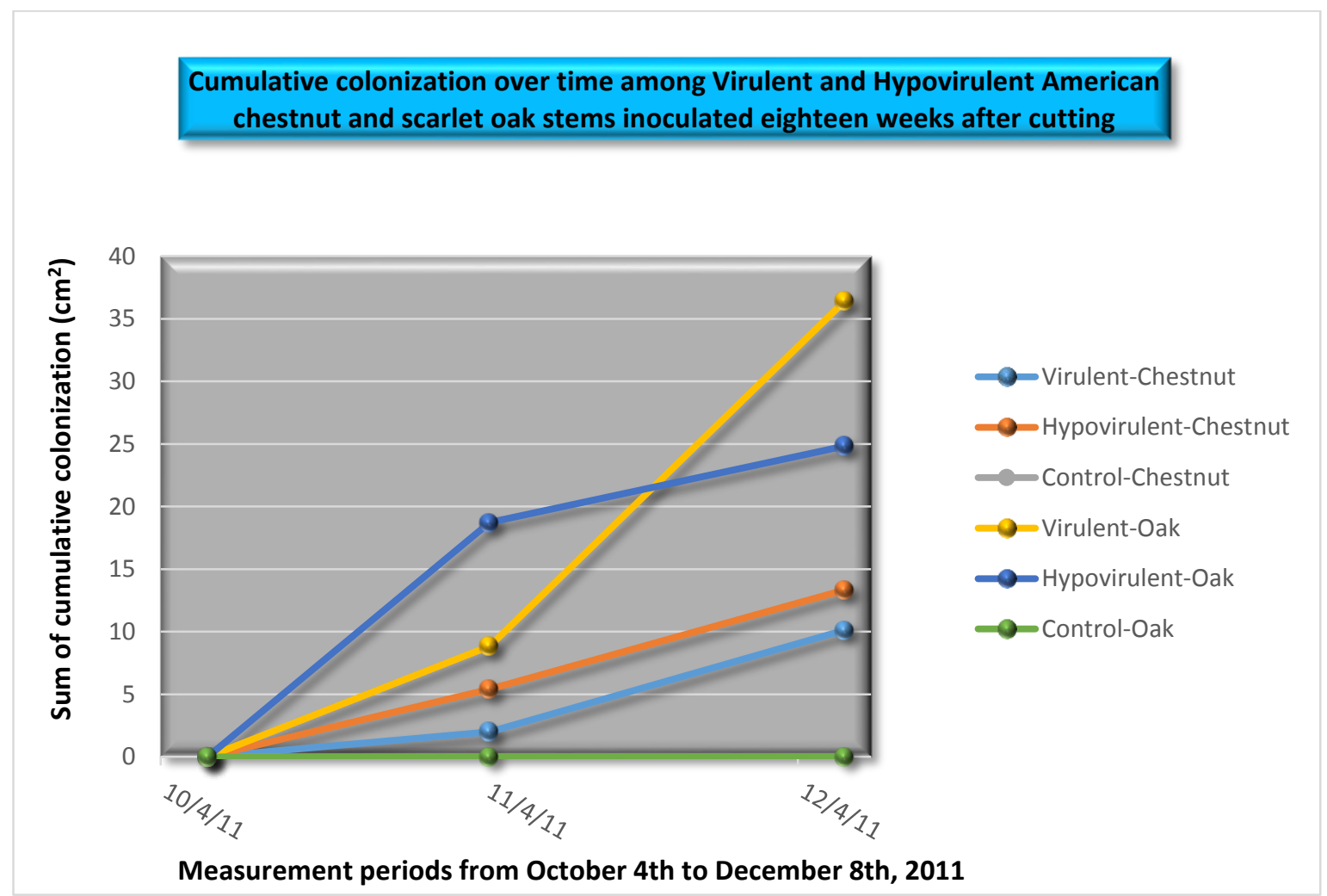

Figure 10: Sum of cumulative colonization for V BRV-1 and HV BRHV-1 were not significantly different at $\alpha=0.05$ when $\mathrm{V}$ and $\mathrm{HV}$ was compared on American chestnut $(\mathrm{P}>\mathrm{F}=<0.3778)$ and the same for scarlet oak $(\mathrm{P}>\mathrm{F}=<0.4255)$ for the third inoculation period from October $4^{\text {th }}$ to December $8^{\text {th }} 2011$. When Control stems were compared with V and HV inoculations, the Control expressed nearly null colonization at the inoculation sites $(\mathrm{P}>\mathrm{F}=0.0001)$. 


\section{SECTION 2: Effect of Layers on Colonization}

\section{First Inoculation Period}

The layers within a stack also were evaluated for differences in bark colonization. This comparison analyzed whether the total fungal colonization was influenced by proximity of the layer to the ground. There was not sufficient evidence to suggest that the mean differences of $\mathrm{V}$ colonization among the layers of a stack of American chestnut were different $(\mathrm{P}>\mathrm{F}=0.2959)$. Even though colonization was measurably higher in the layer (L-1) closest to the ground, it was not significantly so (Figure 11). Likewise for scarlet oak, there was no significant difference for V layer colonization within a stack for the first inoculation period $(\mathrm{P}>\mathrm{F}=0.2954)$ (Figure 12). In the case of scarlet oak, layer colonization was slightly more in L-2.

There also was not sufficient evidence to suggest that the mean difference of HV C. parasitica colonization on the layers within a stack of American chestnut were different $(\mathrm{P}>\mathrm{F}=0.6717)$. In comparison to the $\mathrm{V}$ stack, HV L-1 layer also had the highest mean colonization (Figure 11). Bark colonization means of the inoculated scarlet oak stems by HV C. parasitica were significantly lower on $\mathrm{L}-2$ than the others $(\mathrm{P}>\mathrm{F}=$ 0.0068). Similar to V and HV chestnut, L-1 also had the highest average colonization (Figure 12). 


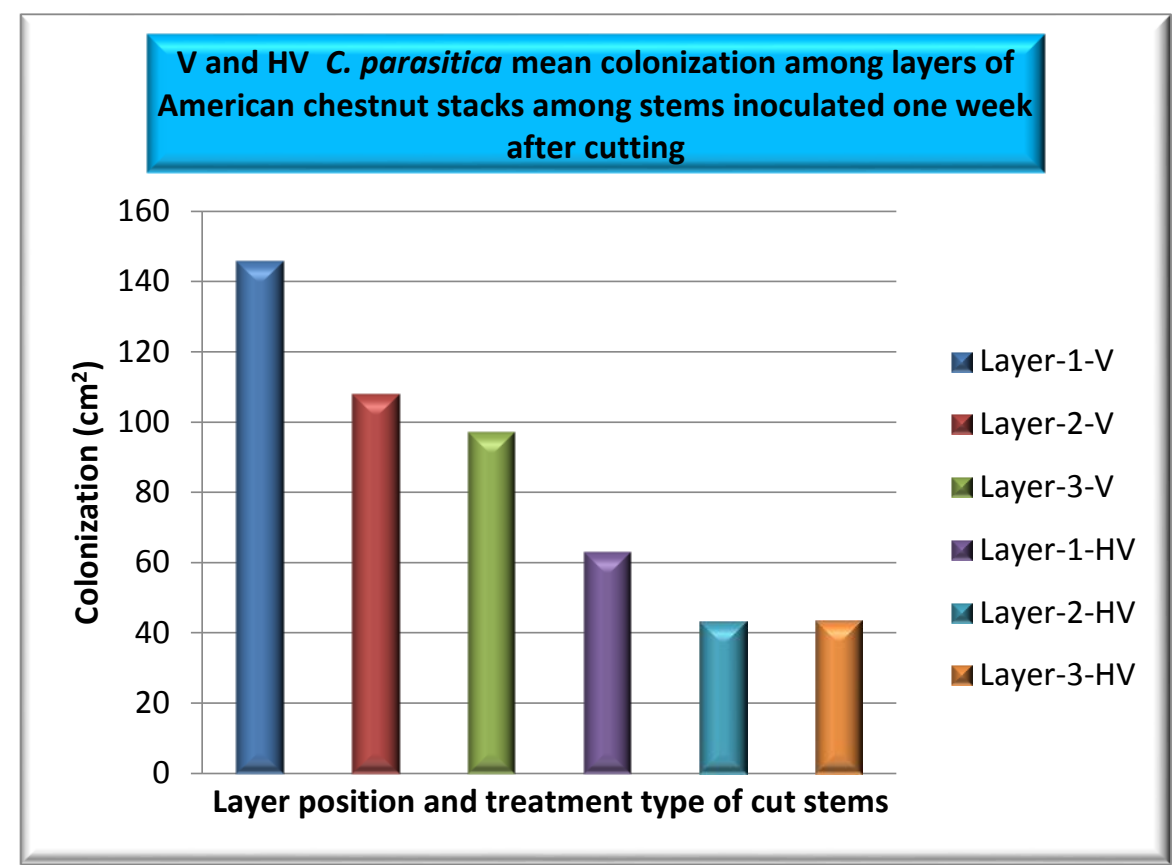

Figure 11: Average colonization for V BRV-1 and HV BRHV-1 not significant respectively at $\alpha=0.05$ $(\mathrm{P}>\mathrm{F}=0.2959)$ and $(\mathrm{P}>\mathrm{F}=0.6717)$ on American chestnut layers L-1 (ground), L-2 (middle) and L-3 (top) for the first inoculation period from May $20^{\text {th }}$ to December $8^{\text {th }} 2011$.

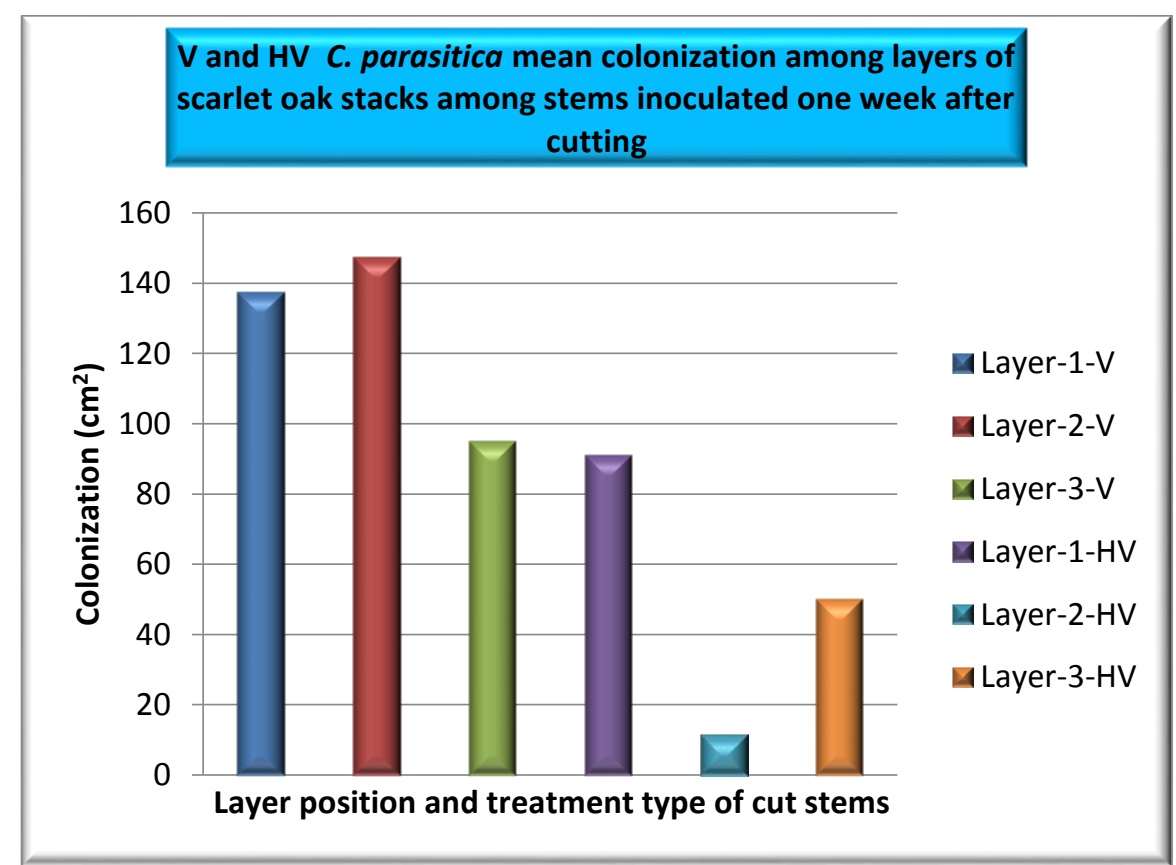

Figure 12: Average colonization for $V B R V-1$ not significant at $\alpha=0.05(P>F=0.2954)$ on scarlet oak layers and HV BRHV-1 significant at $(\mathrm{P}>\mathrm{F}=0.0068)$. L-1 (ground), L-2 (middle) and L-3 (top) for the first inoculation period from May $20^{\text {th }}$ to December $8^{\text {th }} 2011$.

\section{Second Inoculation Period}

As with inoculation period one the mean difference of V C. parasitica mycelial colonization among the layers within a stack of American chestnut $(\mathrm{P}>\mathrm{F}=0.5538)$ or 
scarlet oak $(\mathrm{P}>\mathrm{F}=0.5819)$ for the August $4^{\text {th }}$ inoculation (IP-2) were different (Figures 13 and 14). There also was not sufficient evidence to suggest that the mean difference of HV colonization on the layers within a stack of American chestnut $(\mathrm{P}>\mathrm{F}=0.6768)$ or scarlet oak $(\mathrm{P}>\mathrm{F}=0.4458)$ were different (Figures 13 and 14). All analyses indicated no outstanding colonization differences among any layers within the stacks even though L-2 promoted the most colonization for both the V and HV layers on chestnut and the HV layer on oak.

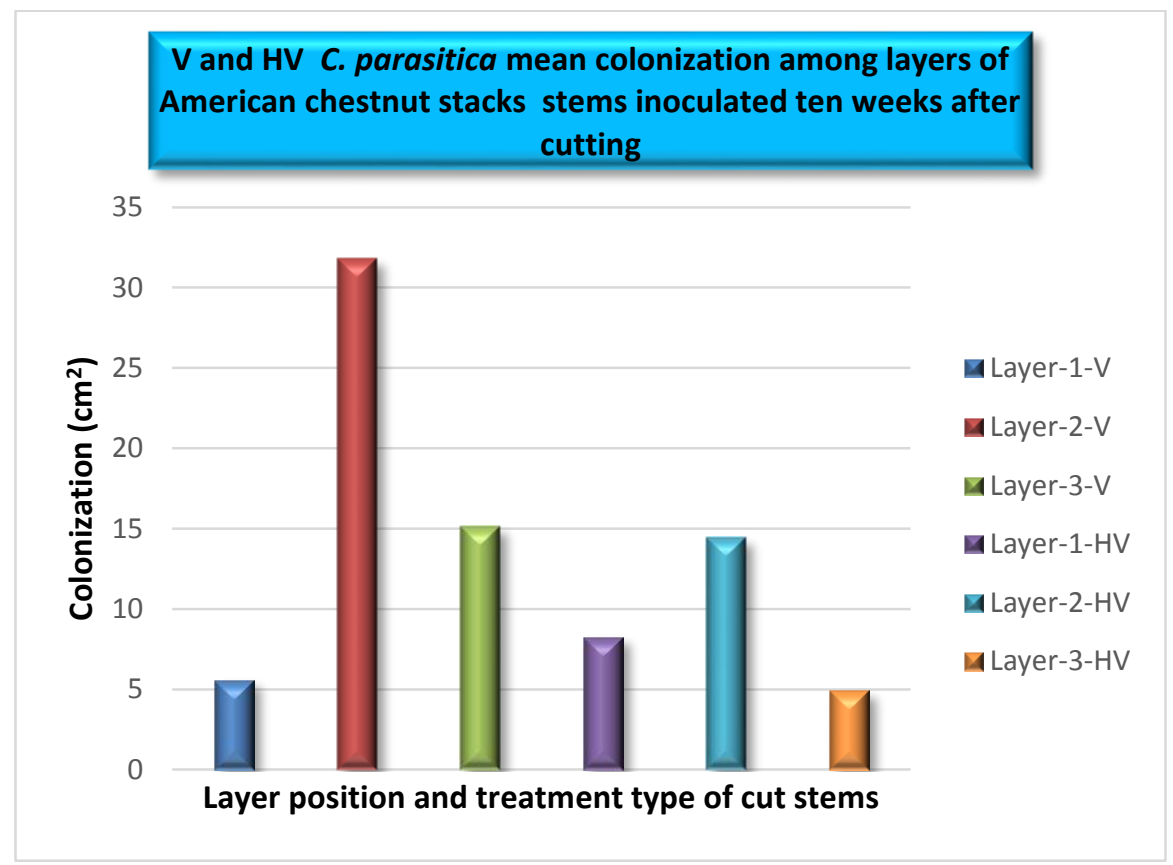

Figure 13: Average colonization for V BRV-1 and HV BRHV-1 not significant, respectively at $\alpha=0.05$ $(\mathrm{P}>\mathrm{F}=0.5538)$ and $(\mathrm{P}>\mathrm{F}=0.6768)$ on American chestnut layers L-1 (ground), L-2 (middle) and L-3 (top) for the second inoculation period from August $4^{\text {th }}$ to December $8^{\text {th }} 2011$. 


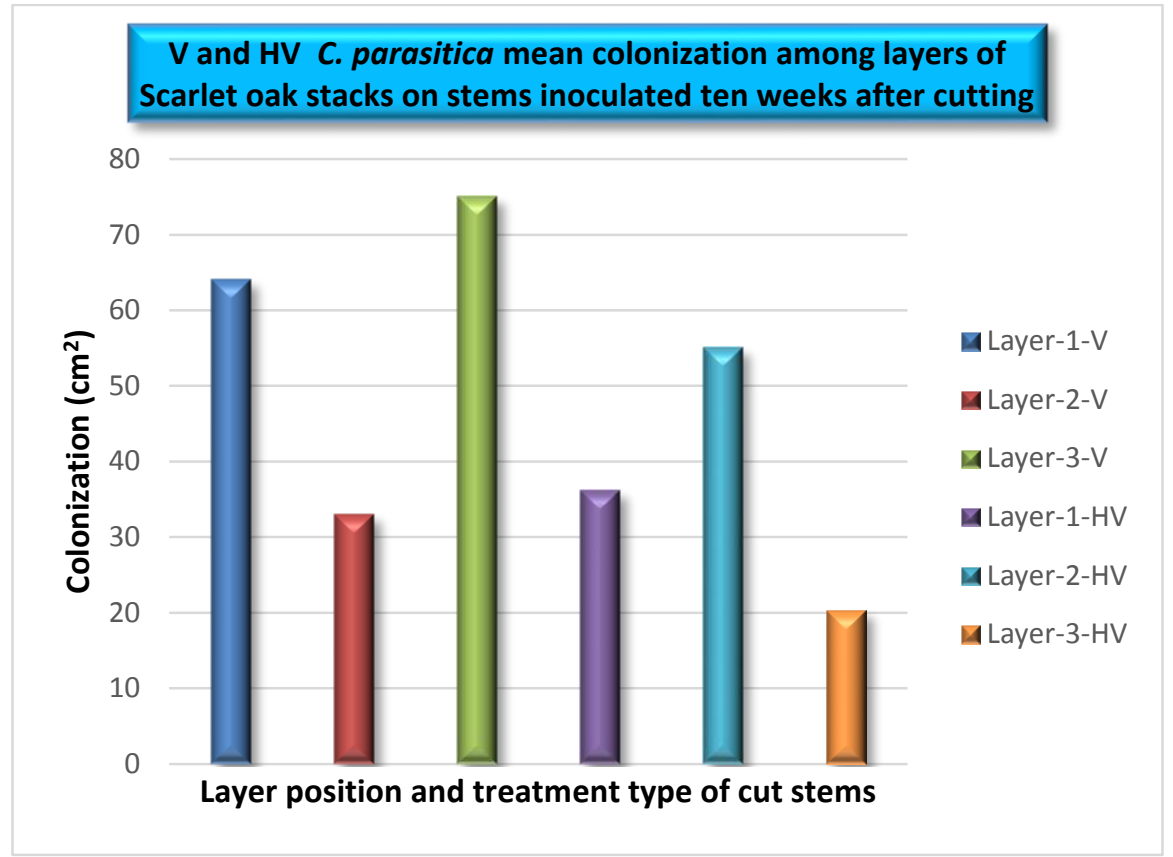

Figure 14: Average colonization for V BRV-1 and HV BRHV-1 not significant, respectively at $\alpha=0.05$ $(\mathrm{P}>\mathrm{F}=0.5819)$ and $(\mathrm{P}>\mathrm{F}=0.4458)$ on scarlet oak layers $\mathrm{L}-1$ (ground), L-2 (middle) and L-3 (top) for the second inoculation period from August $4^{\text {th }}$ to December $8^{\text {th }}$

\section{Third Inoculation Period}

The third inoculation period mirrored the first two in that chestnut and oak were similar. There was not sufficient evidence to suggest that the mean difference of $\mathrm{V}$ colonization on the layers within a stack of American chestnut $(\mathrm{P}>\mathrm{F}=0.2796)$ or scarlet oak $(\mathrm{P}>\mathrm{F}=0.5155)$ for the October $4^{\text {th }}$ inoculation were different (Figures 15 and 16). HV results also were indicative of the previous inoculation periods. Therein, colonization on the layers within a stack of American chestnut $(\mathrm{P}>\mathrm{F}=0.5369)$ and scarlet oak $(\mathrm{P}>\mathrm{F}=0.2131)$ were not different for any layer (Figures 15 and16). Analyses for this period indicated no outstanding colonization differences among any layers within the stacks. 


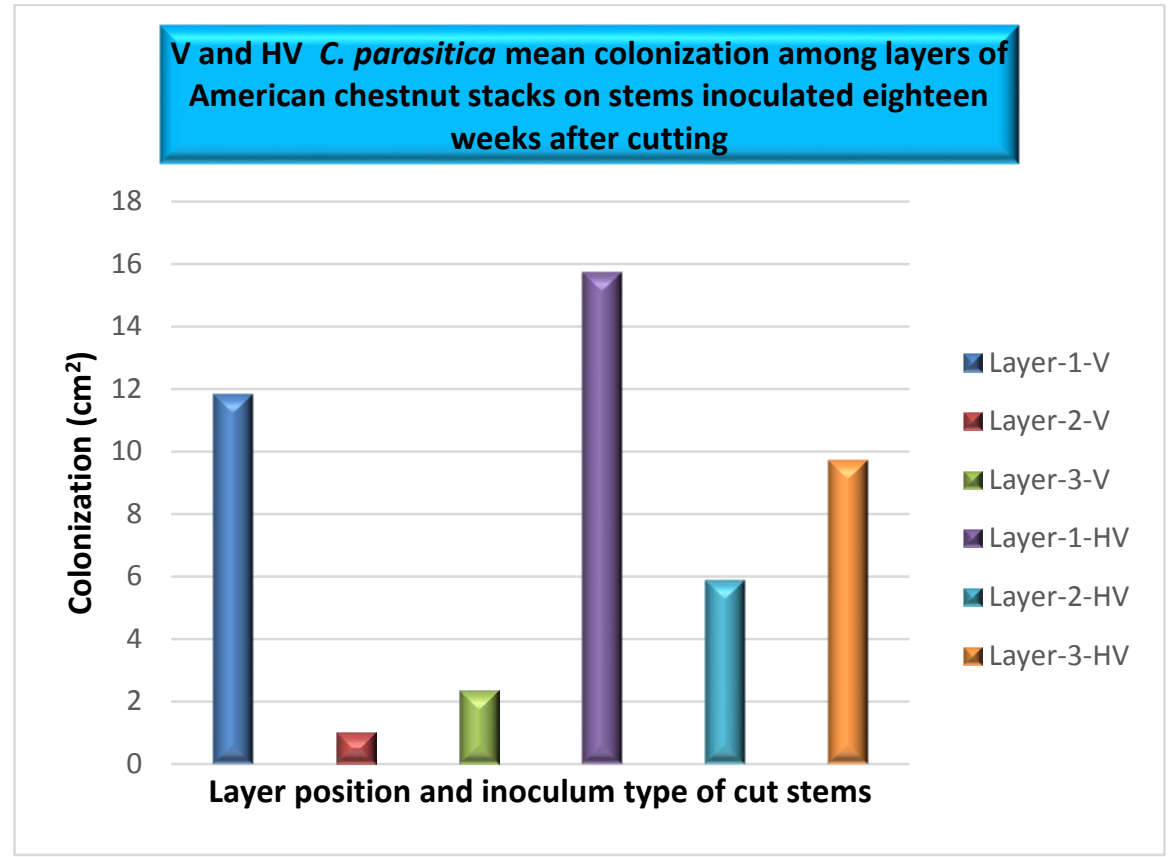

Figure 15: Average colonization for V BRV-1 and HV BRHV-1 not significant, respectively at $\alpha=0.05$ $(\mathrm{P}>\mathrm{F}=0.2796)$ and $(\mathrm{P}>\mathrm{F}=0.5369)$ on American chestnut layers L-1 (ground), L-2 (middle) and L-3 (top) for the third inoculation period from October $4^{\text {th }}$ to December $8^{\text {th }} 2011$.



Figure 16: Average colonization for V BRV-1 and HV BRHV-1 not significant, respectively at $\alpha=0.05$ $(\mathrm{P}>\mathrm{F}=0.5155)$ and $(\mathrm{P}>\mathrm{F}=0.2131)$ on scarlet oak layers L-1 (ground), $\mathrm{L}-2$ (middle) and L-3 (top) for the third inoculation period from October $4^{\text {th }}$ to December $8^{\text {th }} 2011$. 


\section{SECTION 3: Effect of Stack Location on Colonization}

\section{First Inoculation Period}

Analyses were performed to compare the effect of stack location on total

colonization. The oak and chestnut colonization data for a stack receiving the same inoculation type (V, HV and C) were pooled for this analysis. This test analyzed the total colonization of $C$. parasitica can take place within a stack of chestnut and oak. Although groups G-1 through G-5 were spatially distributed, they were all close to one another on a wooded terrace within an area such that 33 meters was the greatest distance between G-1 and G-4. The ANOVA indicated that there was sufficient evidence to suggest colonization differences existed within $\mathrm{V}$ stacks $(\mathrm{P}>\mathrm{F}=0.0532)$. In particular, the colonization for the G-4 stack was significantly less than for G-1 and G-3 (Figure 17).

There also was a statistically significant difference among the HV stacks $(\mathrm{P}>\mathrm{F}=$ 0.0001). All comparisons tests indicated that group G-1 had significantly more colonization than G-2 and G-4 (Figure 18).

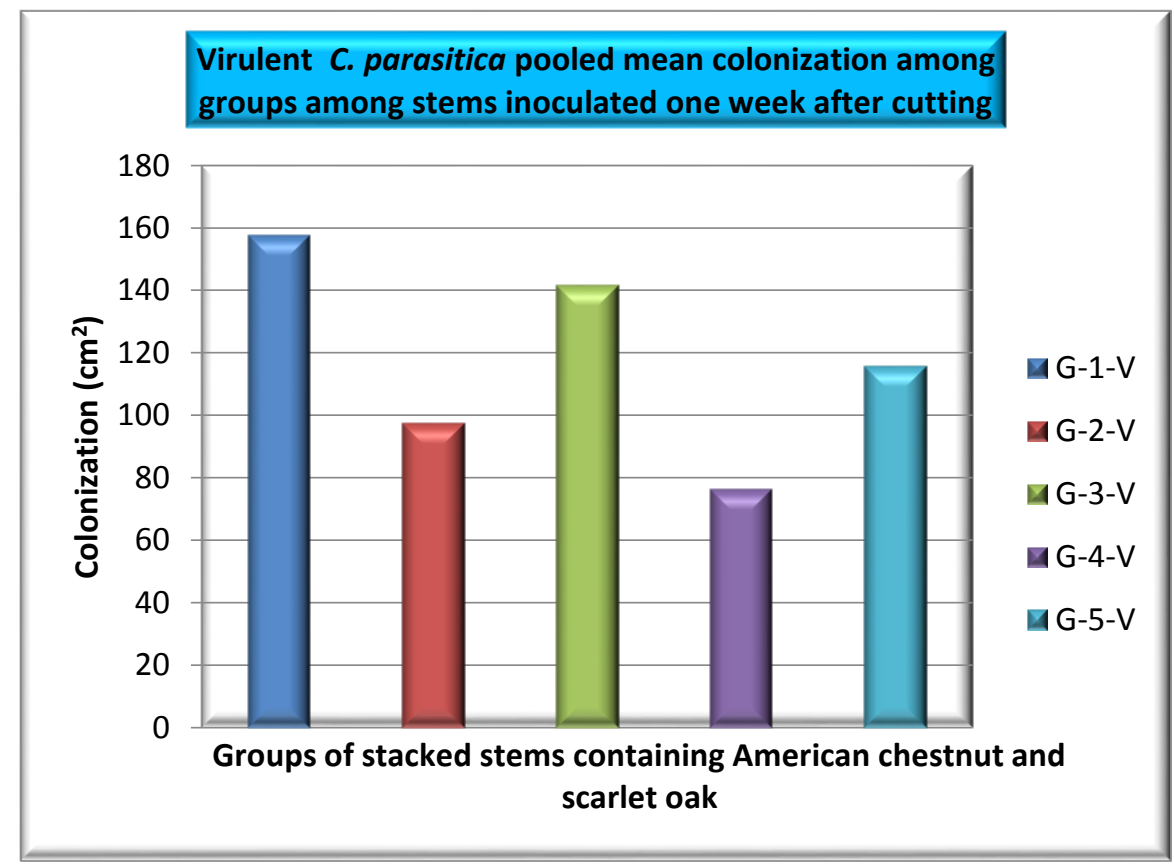

Figure 17: Average colonization for V BRV-1 significant at $\alpha=0.05(\mathrm{P}>\mathrm{F}=0.0532)$ for pooled colonization on American chestnut and scarlet oak within a group for the first inoculation period from May $20^{\text {th }}$ to December $8^{\text {th }} 2011$. 


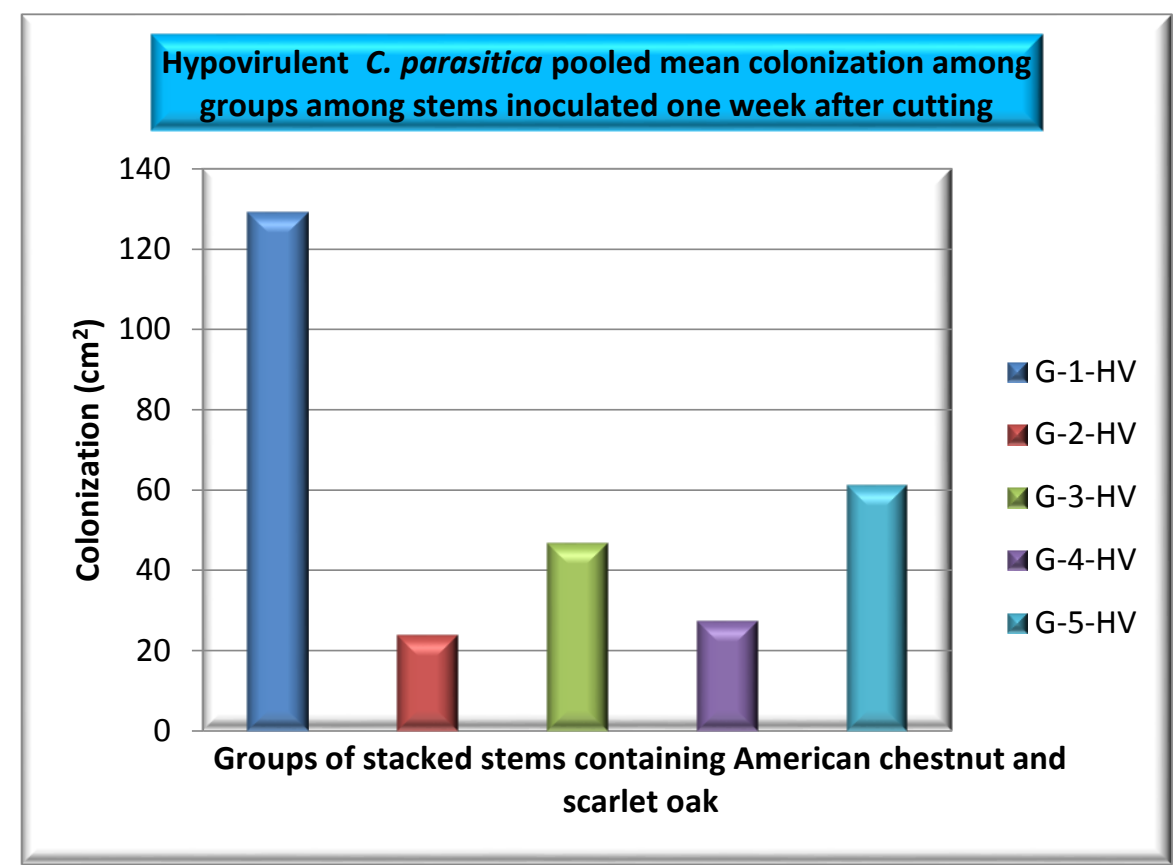

Figure 18: Average colonization for HV BRHV-1 significant at $\alpha=0.05(\mathrm{P}>\mathrm{F}=0.0001)$ for pooled colonization on American chestnut and scarlet oak within a group for the first inoculation period from May $20^{\text {th }}$ to December $8^{\text {th }} 2011$.

\section{Second Inoculation Period}

The ANOVA indicated that there was insufficient evidence to suggest a difference among the stacks colonization for either the $\mathrm{V}(\mathrm{P}>\mathrm{F}=0.7502)$ or $\mathrm{HV}(\mathrm{P}>\mathrm{F}=$ 0.2976) groups. However, in both cases comparing the first and second inoculation periods, G-4 grew the least and G-1 grew the most, but not significantly more than the others (Figures 19 and 20). 


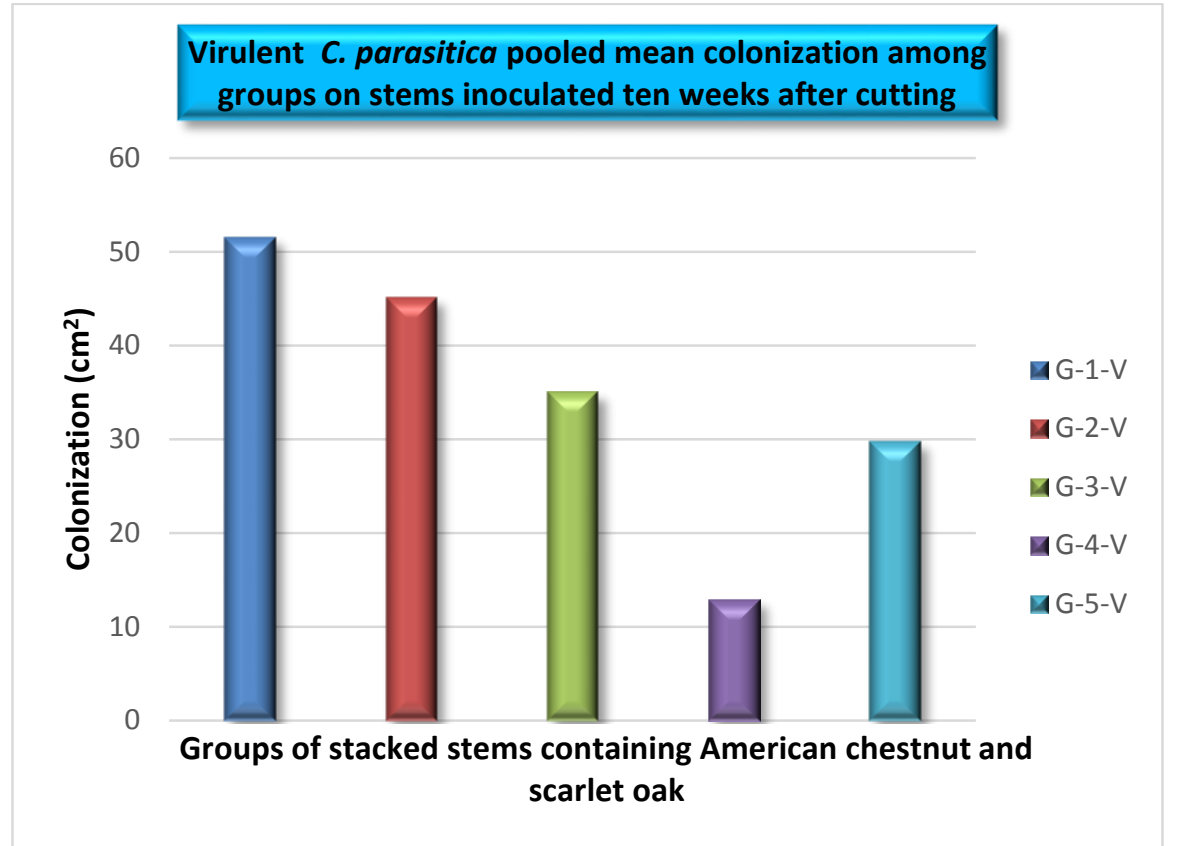

Figure 19: Average colonization for $V$ BRV-1 not significant at $\alpha=0.05(\mathrm{P}>\mathrm{F}=0.7502)$ for pooled colonization on American chestnut and scarlet oak within a group for the second inoculation period from August $4^{\text {th }}$ to December $8^{\text {th }} 2011$.



Figure 20: Average colonization for HV BRHV-1 not significant at $\alpha=0.05(\mathrm{P}>\mathrm{F}=0.2976)$ for pooled colonization on American chestnut and scarlet oak within a group for the second inoculation period from August $4^{\text {th }}$ to December $8^{\text {th }} 2011$. 


\section{Third Inoculation Period}

Again, the ANOVA for this period indicated that there was insufficient evidence to suggest a difference among the stacks for either $\mathrm{V}(\mathrm{P}>\mathrm{F}=0.3815)$ or $\mathrm{HV}(\mathrm{P}>\mathrm{F}$ $=0.7488)$ groups $($ Figures 21 and 22) .

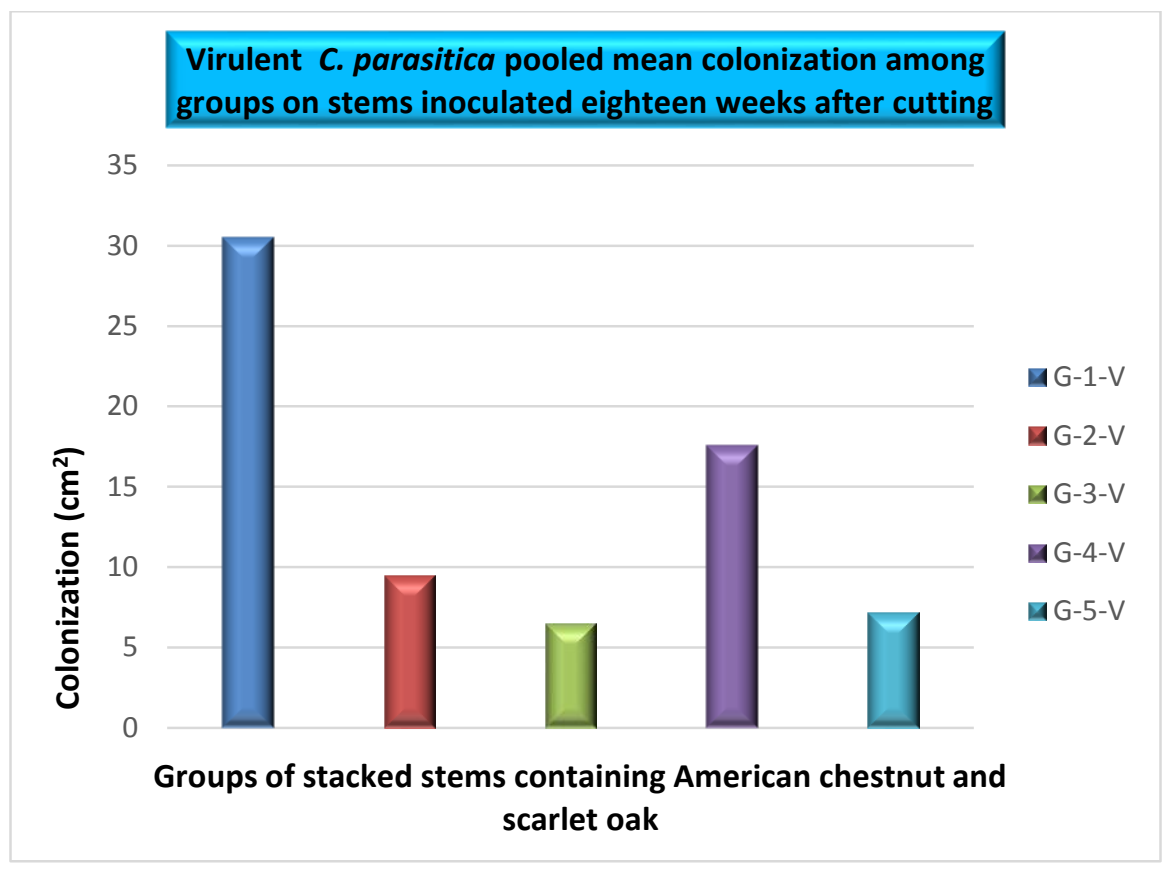

Figure 21: Average colonization for V BRV-1 not significant at $\alpha=0.05(\mathrm{P}>\mathrm{F}=0.7488)$ for pooled colonization on American chestnut and scarlet oak within a group for the third inoculation period from October $4^{\text {th }}$ to December $8^{\text {th }} 2011$.

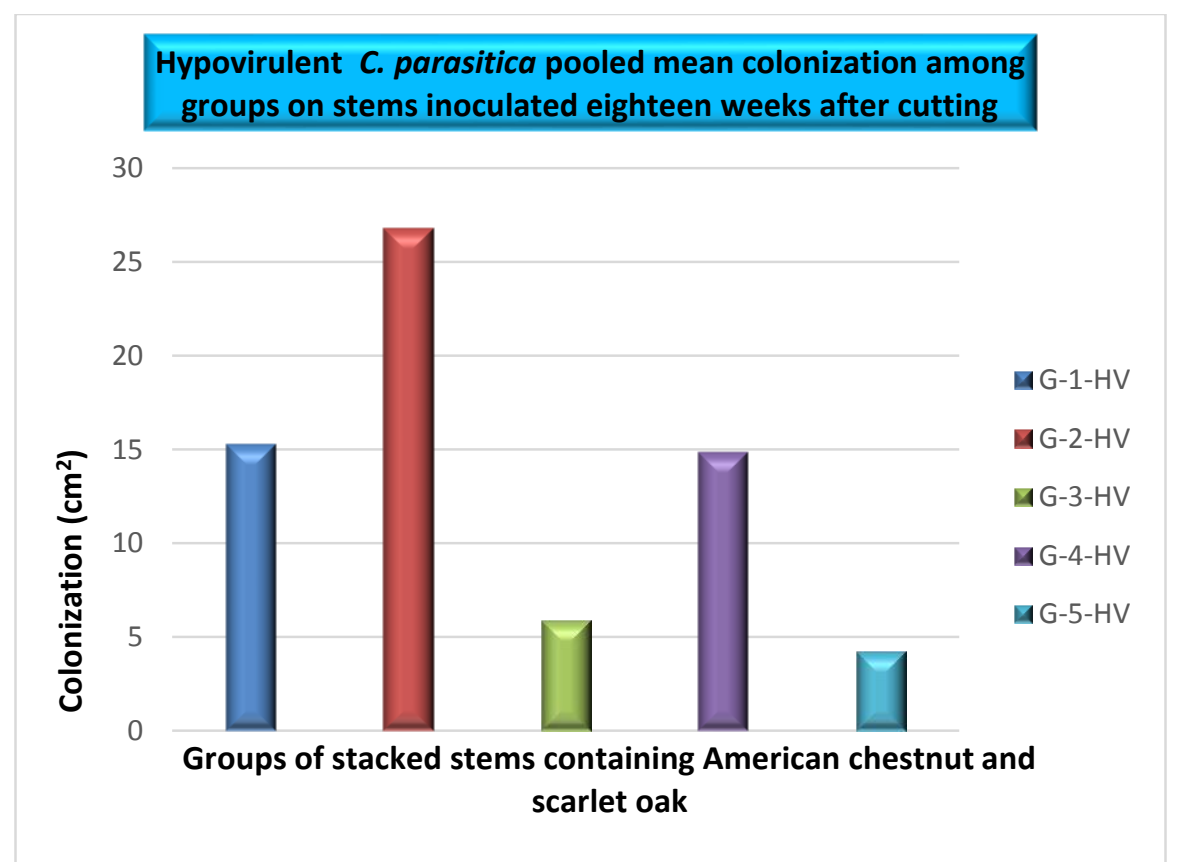

Figure 22: Average colonization for HV BRHV-1 not significant at $\alpha=0.05(\mathrm{P}>\mathrm{F}=0.3815)$ for pooled colonization on American chestnut and scarlet oak within a group for the third inoculation period from October $4^{\text {th }}$ to December $8^{\text {th }} 2011$. 
CHAPTER 1 COLONIZATION SUMMARY: Total Colonization, Effect of Layer and Location of Stack at the Site

Tables 2-4 consolidate the results of bark lesion areas of American chestnut and scarlet oak that were colonized during the May, August and October inoculation periods. Because the y-axis scales differ among the tables and graphs in this chapter, this approach provides a more direct comparison of the data for successive inoculation periods (IP's) on overall colonization, the effect of the layer in which the stem was placed and the effect of the position of the stack at the site. Even though statistical comparisons were not made among the inoculation periods the ability of $C$. parasitica to colonize bark diminished significantly with each successive inoculation period (IP). The data also supports the observation that scarlet oak provided a better substrate for colonization during IP's 2 and 3 then did American chestnut. Other trends indicated higher HV than $\mathrm{V}$ total colonization on chestnut during IP-3. This phenomenon also occurred for layer and location effect. Also, more colonization by V and HV took place during IP-3 than in IP-2 in a few circumstances.

TABLE 2: Average $C$. parasitica total colonization measurement comparisons for American chestnut and scarlet oak including all treatments and inoculation periods.

\begin{tabular}{|l|l|l|l|l|l|l|}
\hline $\begin{array}{l}\text { Total } \\
\text { Colonization }\end{array}$ & Inoc $\mathbf{1}$ & \multicolumn{2}{|l|}{ Inoc 2 } & Inoc 3 \\
\hline & Cd-V & $\begin{array}{l}\text { Colonization } \\
\left(\mathrm{cm}^{2}\right)\end{array}$ & \multicolumn{2}{|l|}{$\begin{array}{l}\text { Colonization } \\
\left(\mathrm{cm}^{2}\right)\end{array}$} & $\begin{array}{l}\text { Colonization } \\
\left(\mathrm{cm}^{2}\right)\end{array}$ \\
\hline Qc-V & 111 & & 18 & $6^{\mathrm{c}}$ \\
\hline Cd-HV & 124 & & 52 & 22 \\
\hline Qc-HV & 55 & & 9 & 9 \\
\hline Control $^{\mathbf{b}}$ & 67 & & 37 & 17 \\
\hline
\end{tabular}

${ }^{\text {a }} \mathrm{Cd}=$ Castanea dentata $; \mathrm{Qc}=$ Quercus coccinea

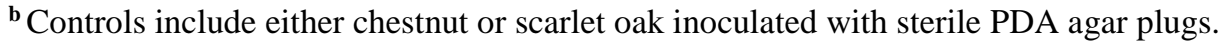

${ }^{\mathbf{c}}$ Yellow highlighted text indicates that HV grew more than V within the same tree species. 
TABLE 3: Average $C$. parasitica layer effect colonization measurement comparisons for American chestnut and scarlet oak including all treatments and inoculation periods.

\begin{tabular}{|c|c|c|c|}
\hline $\begin{array}{l}\text { Layer } \\
\text { Colonizationa }\end{array}$ & Inoc 1 & Inoc 2 & Inoc 3 \\
\hline & $\begin{array}{l}\text { Colonization } \\
\left(\mathrm{cm}^{2}\right)\end{array}$ & $\begin{array}{l}\text { Colonization } \\
\left(\mathrm{cm}^{2}\right)\end{array}$ & $\begin{array}{l}\text { Colonization } \\
\left(\mathrm{cm}^{2}\right)\end{array}$ \\
\hline Cd-L1-V & \begin{tabular}{|l|l|}
146 \\
\end{tabular} & \begin{tabular}{l|l}
$6^{c}$ \\
\end{tabular} & 11 \\
\hline Cd-L2-V & 108 & 15 & 2 \\
\hline Cd-L3-V & 97 & 15 & 6 \\
\hline Cd-L1-HV & 63 & 32 & 1 \\
\hline Cd-L2-HV & 43 & 8 & 16 \\
\hline Cd-L3-HV & 43 & 5 & 9 \\
\hline Qc-L1-V & 137 & 64 & 5 \\
\hline Qc-L2-V & 147 & 75 & 35 \\
\hline Qc-L3-V & 94 & 55 & 4 \\
\hline Qc-L1-HV & 90 & 33 & 34 \\
\hline Qc-L2-HV & $11^{\mathrm{d}}$ & 36 & 14 \\
\hline Qc-L3-HV & 50 & 20 & 32 \\
\hline Control $^{b}$ & 0 & 0 & 0 \\
\hline
\end{tabular}

${ }^{\mathrm{a}} \mathrm{Cd}=$ Castanea dentata $; \mathrm{Qc}=$ Quercus coccinea

${ }^{\mathrm{b}}$ Controls include either chestnut or scarlet oak inoculated with sterile PDA agar plugs.

${ }^{c}$ Yellow highlighted text indicates that HV grew more than $\mathrm{V}$ within the same tree species and layer type.

d Red blocks indicate a region where the subsequent IP's colonization was greater than the previous IP's.

TABLE 4: Average $C$. parasitica location effect colonization measurement comparisons for American chestnut and scarlet oak including all treatments and inoculation periods.

\begin{tabular}{|l|l|l|l|l|l|}
\hline $\begin{array}{l}\text { Group } \\
\text { Colonization }\end{array}$ & Inoc 1 & & Inoc 2 & Inoc 3 \\
\hline & $\begin{array}{l}\text { Colonization } \\
\left(\mathrm{cm}^{2}\right)\end{array}$ & $\begin{array}{l}\text { Colonization } \\
\left(\mathrm{cm}^{2}\right)\end{array}$ & $\begin{array}{l}\text { Colonization } \\
\left(\mathrm{cm}^{2}\right)\end{array}$ \\
\hline G1-V & 158 & & 51 & 31 \\
\hline G2-V & 97 & & 45 & 10 \\
\hline G3-V & 141 & & 35 & 7 \\
\hline G4-V & 76 & & 13 & 17 \\
\hline G5-V & 116 & & 30 & 7 \\
\hline G1-HV & 129 & & 50 & 15 \\
\hline G2-HV & 24 & & 20 & 27 \\
\hline G3-HV & 47 & & 22 & 6 \\
\hline G4-HV & 27 & & 4 & 15 \\
\hline G5-HV & 61 & & 21 & 4 \\
\hline Control & 0 & & 0 & 0 \\
\hline
\end{tabular}

${ }^{a}$ Controls include either chestnut or scarlet oak inoculated with sterile PDA agar plugs.

${ }^{\mathbf{b}}$ Represents total combined Group colonization within full, mixed stem stacks of $C$. dentata and $Q$. coccinea

${ }^{\mathbf{c}}$ Yellow highlighted text indicates that HV grew more than $\mathrm{V}$ within the same tree species and layer type.

d Red blocks indicate a region where the subsequent IP's colonization was greater than the previous IP's. 


\section{CHAPTER 2: SPORULATION}

\section{SECTION 1: Analysis of Total Sporulation}

The same three inoculation periods were used to assess sporulation of $C$.

parasitica on the American chestnut and scarlet oak stems. A visual assessment was used to evaluate the differences in stroma formation and density on the inoculated $\mathrm{V}$ and HV stems. The first analysis compared the presence of stroma for V and HV on the bark of the two species during the three inoculation periods. A second analysis considered whether position in the stack had an effect on stroma formation and the third evaluated whether differences existed among groups due to their location at the site. Ordinal sporulation ranks were pooled from throughout the course of each inoculation period to assess total sporulation over the entire inoculation period duration. Sporulation ranking results were analyzed by a one way ANOVA using $\alpha=0.05$.

\section{First Inoculation Period}

The V strain sporulated much more on chestnut than on oak for the first three months $(\mathrm{P}>\mathrm{F}=0.0001)$. However, by October $4^{\text {th }}$, the virulent fungus sporulation on the two species was not significantly different between the two species and remained so for the balance of the experiment ( $\mathrm{P}>\mathrm{F}=<0.09800$ to 0.4294$)$ (Figure 23).

When HV mean sporulation was evaluated, American chestnut also was initially significantly higher than scarlet oak $(\mathrm{P}>\mathrm{F}=0.0001)$. However, similar to $\mathrm{V}$, by October $4^{\text {th }}$, the HV fungus sporulation was not significantly different and remained so for the duration of the first inoculation period ( $\mathrm{P}>\mathrm{F}=<0.1543$ to 0.0834$)$ (Figure 23). In general, C. parasitica sporulated relatively as well on both species over time when inoculated within a week of cutting. 
Sporulation by the V strain was significantly greater than HV for both and chestnut and oak. $(\mathrm{P}>\mathrm{F}=0.0001)$ (Figure 24). The average sporulation at each sampling period for the third inoculation period treatments is illustrated in Figure 24.

Overall, V and HV had the greatest average sporulation on American chestnut. Additionally, HV chestnut sporulated better than V oak. The water agar inoculated control stems showed almost no sporulation compared to the inoculated stems and expressed essentially null sporulation at the established inoculation points $(\mathrm{P}>\mathrm{F}=$ $<0.0001$ ) (Figure 24). Some infections occurred at the cut ends of the stems but were not considered in the experiment.

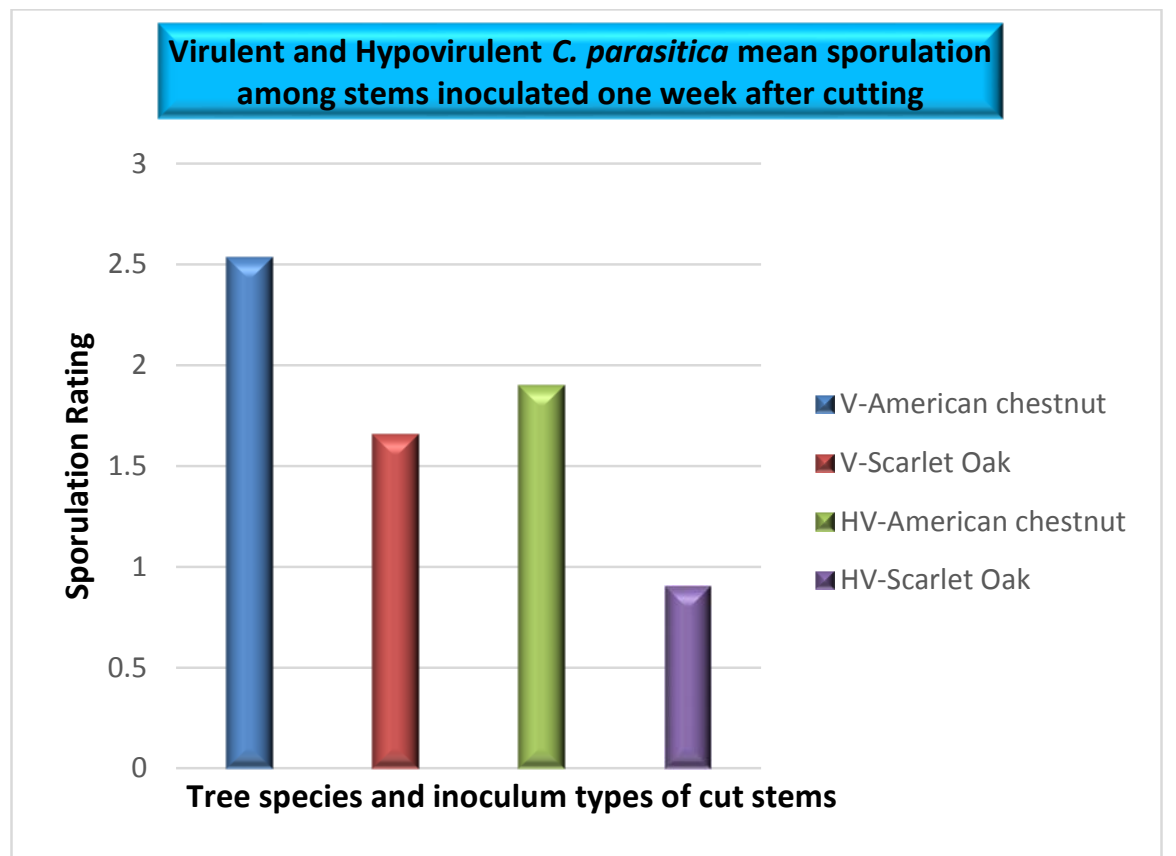

Figure 23: Average sporulation for V BRV-1 and HV BRHV-1 not significantly different between American chestnut and scarlet oak at $\alpha=0.05(\mathrm{P}>\mathrm{F}=0.4294)$ and $(\mathrm{P}>\mathrm{F}=0.0834)$ respectively, for the first inoculation period from May $20^{\text {th }}$ to December $8^{\text {th }} 2011$. 


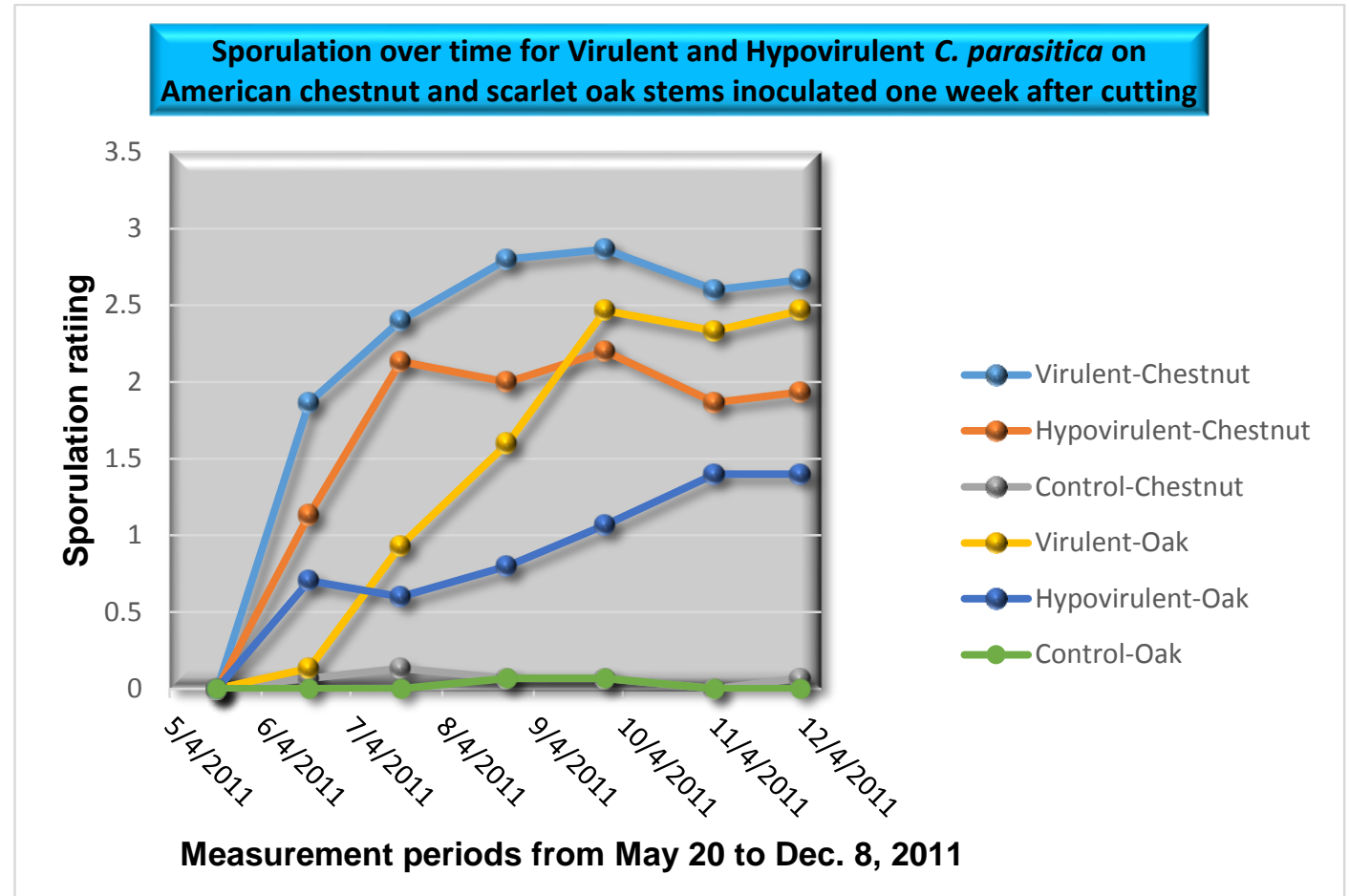

Figure 24: Average sporulation for V BRV-1 and HV BRHV-1 were significantly different at $\alpha=0.05$ ( $P>F$ $=0.0001$ ) when $\mathrm{V}$ and $\mathrm{HV}$ was compared on American chestnut and the same for scarlet oak for the first inoculation period from May $20^{\text {th }}$ to December $8^{\text {th }}$ 2011. When Control stems were compared with $\mathrm{V}$ and $\mathrm{HV}$ inoculations, the Control expressed nearly null sporulation at the inoculation sites $(\mathrm{P}>\mathrm{F}=0.0001)$.

\section{Second Inoculation Period}

The $\mathrm{V}$ inoculations sporulated somewhat equally initially and then oak began sporulating significantly more than chestnut by the October, $4^{\text {th }}$ assessment $(\mathrm{P}>\mathrm{F}=$ $<0.0521)$. The duration of the inoculation period showed that the $\mathrm{V}$ isolate yielded no statistically different results between the two species $(\mathrm{P}>\mathrm{F}=0.1585)$ (Figure 25). However, the V isolate's sporulation developed more on oak than chestnut.

When HV mean sporulation was evaluated, American chestnut stems exhibited significantly less sporulation than scarlet oak $(\mathrm{P}>\mathrm{F}=0.0028)$ (Figure 25). Similar to the sporulation of the V strain, the HV fungus produced more stroma on oak. Cryphonectria parasitica sporulation on American chestnut remained less than that of scarlet oak for both $\mathrm{V}$ and $\mathrm{HV}$ inoculum types for the balance of this inoculation period.

In contrast to the first inoculation period, $\mathrm{V}$ sporulation was not significantly greater than HV for either chestnut $(\mathrm{P}>\mathrm{F}=0.6283)$ or oak $(\mathrm{P}>\mathrm{F}=0.8635)$ (Figure 26). 
The average sporulation at each sampling period for the third inoculation period treatments is illustrated in Figure 26.

The water agar inoculated control stems showed almost no sporulation and expressed essentially null sporulation at the established inoculation points $(\mathrm{P}>\mathrm{F}=$ $<0.0001$ ) (Figure 26). Some infections occurred at the cut ends of the stems but were not considered in the experimental data set.

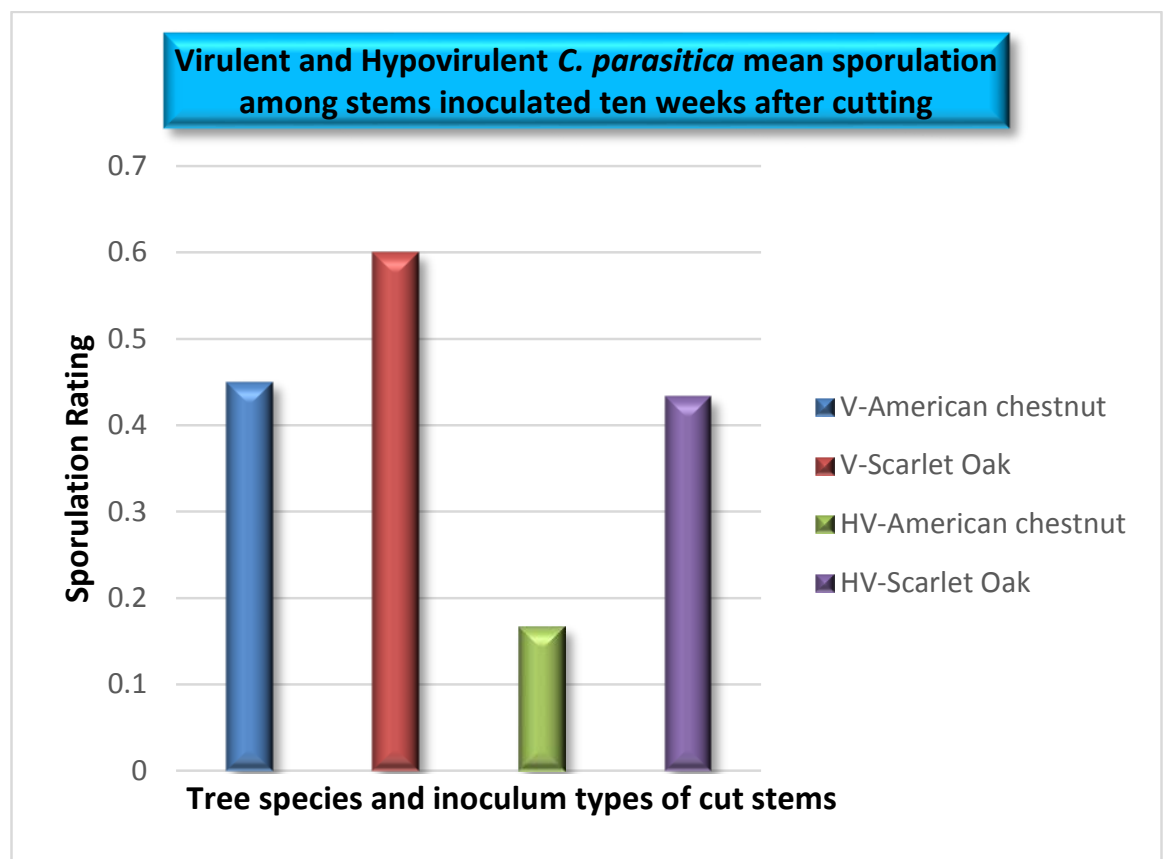

Figure 25: Average sporulation for V BRV-1 and HV BRHV-1 between scarlet oak and American chestnut not significantly different for $\mathrm{V}$ at $\alpha=0.05(\mathrm{P}>\mathrm{F}=0.1585)$ and significantly different on $\mathrm{HV}(\mathrm{P}>\mathrm{F}=0.0028)$ respectively, for the second inoculation period from August $4^{\text {th }}$ to December $8^{\text {th }} 2011$. 


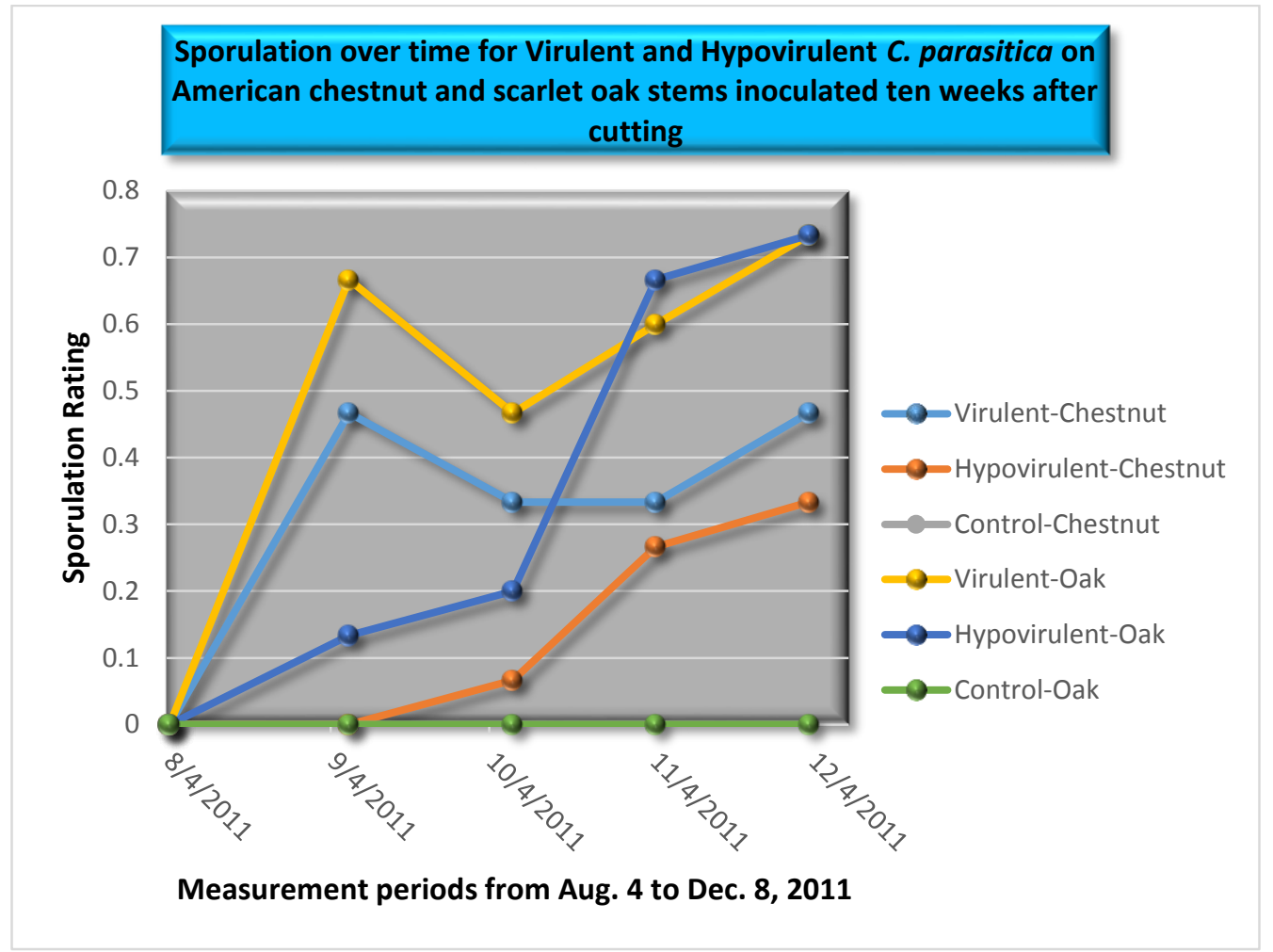

Figure 26: Average sporulation for $\mathrm{V} B R \mathrm{~B}-1$ and HV BRHV-1 were not significantly different at $\alpha=0.05$ when $\mathrm{V}$ and $\mathrm{HV}$ was compared on American chestnut $(\mathrm{P}>\mathrm{F}=0.6283)$ and the same for scarlet oak $(\mathrm{P}>\mathrm{F}=$ $0.8635)$ for the second inoculation period from August $4^{\text {th }}$ to December $8^{\text {th }} 2011$. When Control stems were compared with $\mathrm{V}$ and $\mathrm{HV}$ inoculations, the Control expressed nearly null sporulation at the inoculation sites $(\mathrm{P}>\mathrm{F}=0.0001)$.

\section{Third Inoculation Period}

For the third set of inoculations, initiated on October $4^{\text {th }}$, V sporulation was not statistically different for either species $(\mathrm{P}>\mathrm{F}=0.2999)$ (Figure 27). However, visually the virulent strain tended to sporulate more on oak.

When HV mean sporulation was evaluated between the two species they were numerically the same $(\mathrm{P}>\mathrm{F}=1.0000)$ (Figure 27). The comparison of $\mathrm{V}$ to $\mathrm{HV}$ for chestnut $(\mathrm{P}>\mathrm{F}=0.5311)$ and oak $(\mathrm{P}>\mathrm{F}=0.8115)$ showed no significant sporulation differences (Figure 28). The average sporulation at each sampling period for the third inoculation period treatments is illustrated in Figure 28.

The water agar inoculated control stems showed almost no sporulation compared to the inoculated stems and expressed essentially null stroma formation at the established 
inoculation points $(\mathrm{P}>\mathrm{F}=<0.0001)$ (Figure 28). Some infections occurred at the cut

ends of the stems but were not considered in the experimental data set.

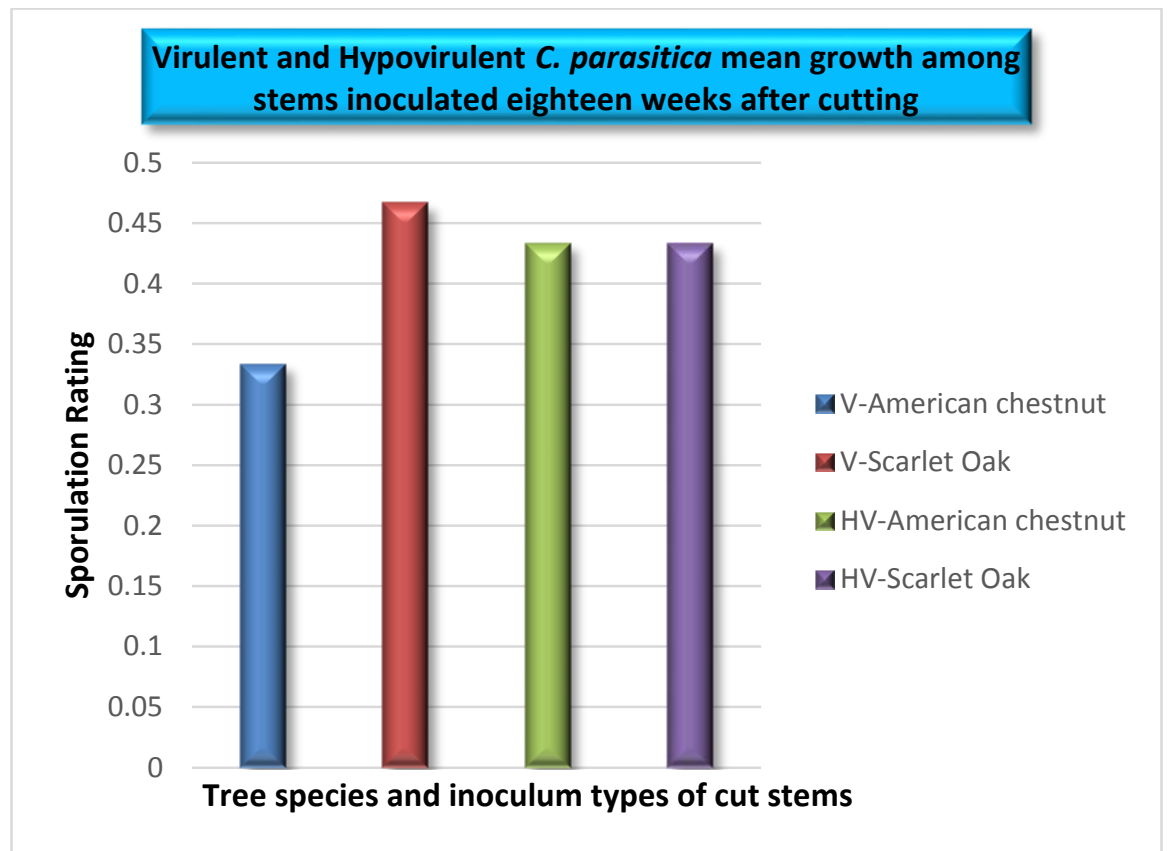

Figure 27: Average sporulation for V BRV-1 and HV BRHV-1 not significantly different between scarlet oak and American chestnut for $\mathrm{V}$ at $\alpha=0.05(\mathrm{P}>\mathrm{F}=0.2999)$ or $\mathrm{HV}(\mathrm{P}>\mathrm{F}=1.0000)$ respectively, for the third inoculation period from October $4^{\text {th }}$ to December $8^{\text {th }} 2011$.

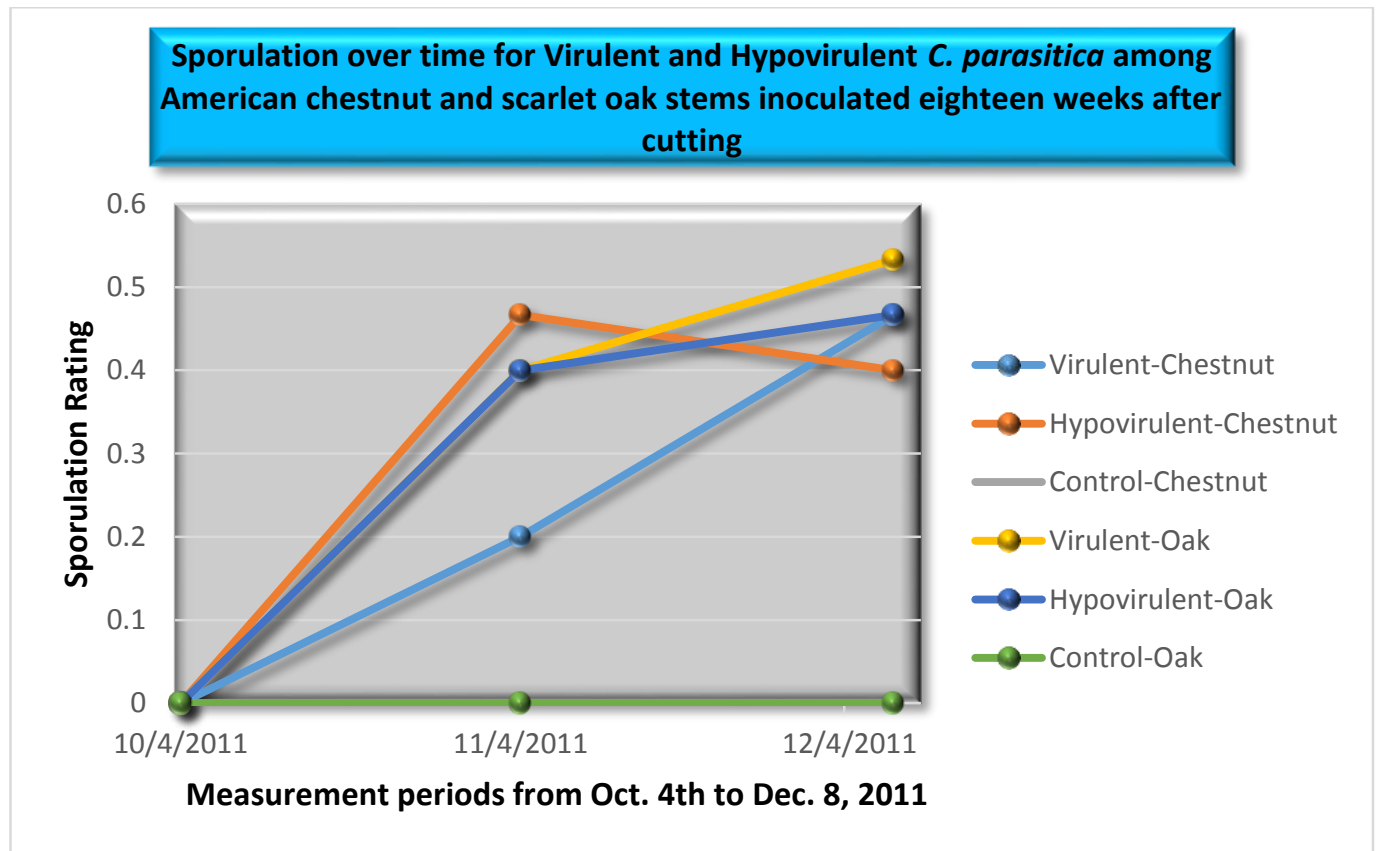

Figure 28: Average sporulation for V BRV-1 and HV BRHV-1 were not significantly different at $\alpha=0.05$ when $\mathrm{V}$ and $\mathrm{HV}$ was compared on American chestnut $(\mathrm{P}>\mathrm{F}=0.5311)$ and the same for scarlet oak $(\mathrm{P}>\mathrm{F}=$ $0.8115)$ for the third inoculation period from October $4^{\text {th }}$ to December $8^{\text {th }} 2011$. When Control stems were compared with $\mathrm{V}$ and $\mathrm{HV}$ inoculations, the Control expressed nearly null sporulation at the inoculation sites $(\mathrm{P}>\mathrm{F}=0.0001)$. 


\section{SECTION 2: Effect of Layers on Sporulation}

\section{First Inoculation Period}

The layers within a stack were evaluated for differences in sporulation. This comparison analyzed whether sporulation was influenced by distance of the layer from the ground. There was no statistical evidence to suggest that the mean difference of $\mathrm{V} C$. parasitica sporulation on the layers within a stack of American chestnut were different However, total sporulation was visually higher in the layer (L-1) closest to the ground, but not significantly so $(\mathrm{P}>\mathrm{F}=0.0870)$ (Figure 29). There was a significant difference between V sporulation on scarlet oak layers within a stack for the May inoculation period where L-1 sporulated more than L-2 $(\mathrm{P}>\mathrm{F}=0.0313)$ (Figure 30).

There also was sufficient evidence to suggest that the mean difference of HV $C$. parasitica sporulation on the layers within a stack of American chestnut were different, where L-2 developed fewer stroma than L-1 and L-3 $(\mathrm{P}>\mathrm{F}=0.0006)$. In contrast to $\mathrm{V}$, the HV L-3 layer had the highest mean sporulation (Figure 29). Sporulation means on the inoculated scarlet oak stems by HV C. parasitica was significantly lower on L-2 than the other two layers $(\mathrm{P}>\mathrm{F}=0.0054)$. The $\mathrm{HV}$ treatments for both oak and chestnut showed that L-3 had the highest average sporulation (Figure 30). 


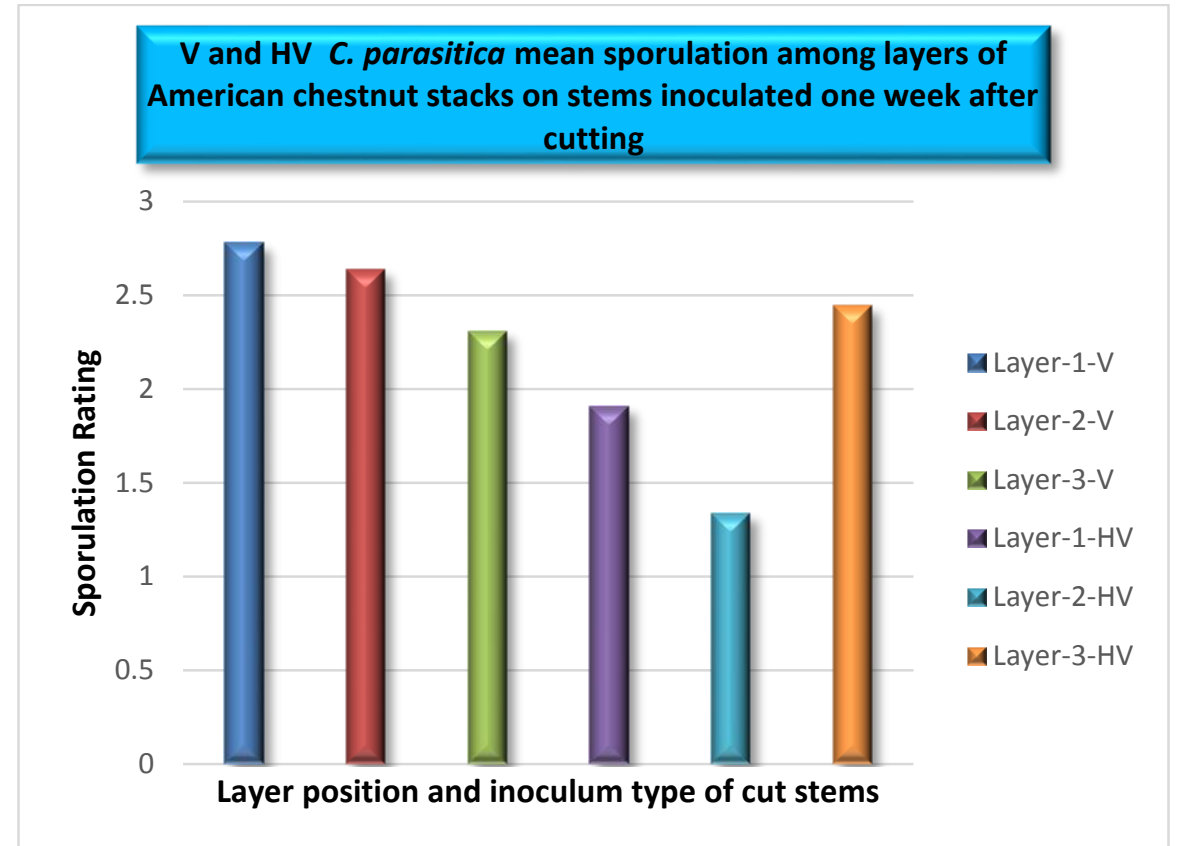

Figure 29: Average sporulation for V BRV-1 and HV BRHV-1 not significant for $\mathrm{V}$ at $\alpha=0.05$ ( $\mathrm{P}>\mathrm{F}=$ $0.0870)$ and significant for $\mathrm{HV}(\mathrm{P}>\mathrm{F}=0.0006)$ on American chestnut layers. L-1 (ground), L-2 (middle) and L-3 (top) for the first inoculation period from May $20^{\text {th }}$ to December $8^{\text {th }} 2011$.

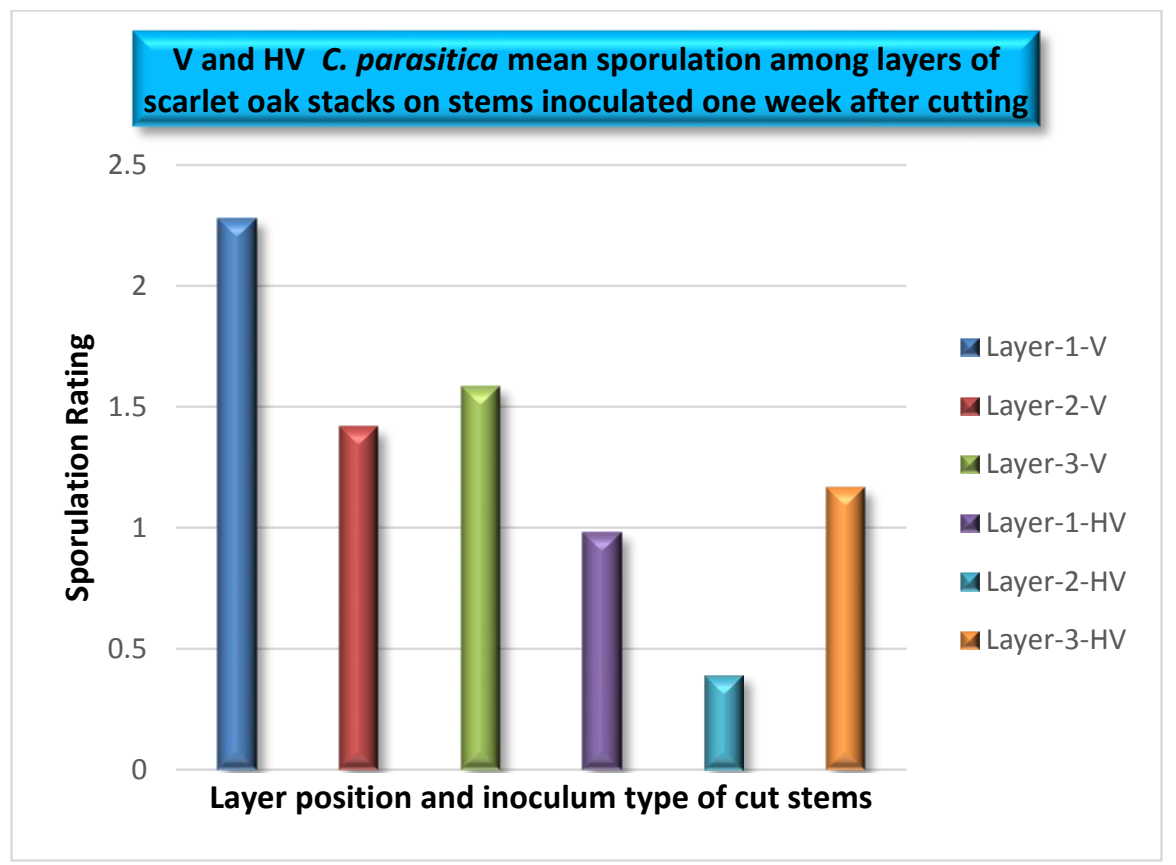

Figure 30: Average sporulation for V BRV-1 and HV BRHV-1 significant at $\alpha=0.05(\mathrm{P}>\mathrm{F}=0.0313)$ and $(\mathrm{P}>\mathrm{F}=0.0054)$ on scarlet oak layers, respectively. L-1 (ground), L-2 (middle) and L-3 (top) for the first inoculation period from May $20^{\text {th }}$ to December $8^{\text {th }} 2011$. 


\section{Second Inoculation Period}

There was not sufficient evidence to suggest that the mean difference of $\mathrm{V} C$.

parasitica sporulation on the layers within a stack of American chestnut were different $(\mathrm{P}>\mathrm{F}=0.9476)($ Figure 31). There was a significant difference between $\mathrm{V}$ sporulation on scarlet oak layers for this inoculation period, where, L-3 sporulated greater than L-2 ( $P>F$ $=0.0169)($ Figure 32).

There also was no statistical evidence that the mean difference of HV $C$.

parasitica sporulation on the layers within a stack of American chestnut were different $(\mathrm{P}>\mathrm{F}=0.6500)$ (Figure 31). However, Figure 31 shows that L-1 sporulation was slightly higher than the other layers. A trend did exist with sporulation decreasing from L-1 to L3 for both V and HV strains. Bark sporulation means of the inoculated scarlet oak stems by $\mathrm{HV}$ also were not significantly different within any layer $(\mathrm{P}>\mathrm{F}=0.4448)$ though, the

L-1 layer had the lowest average sporulation (Figure 32).

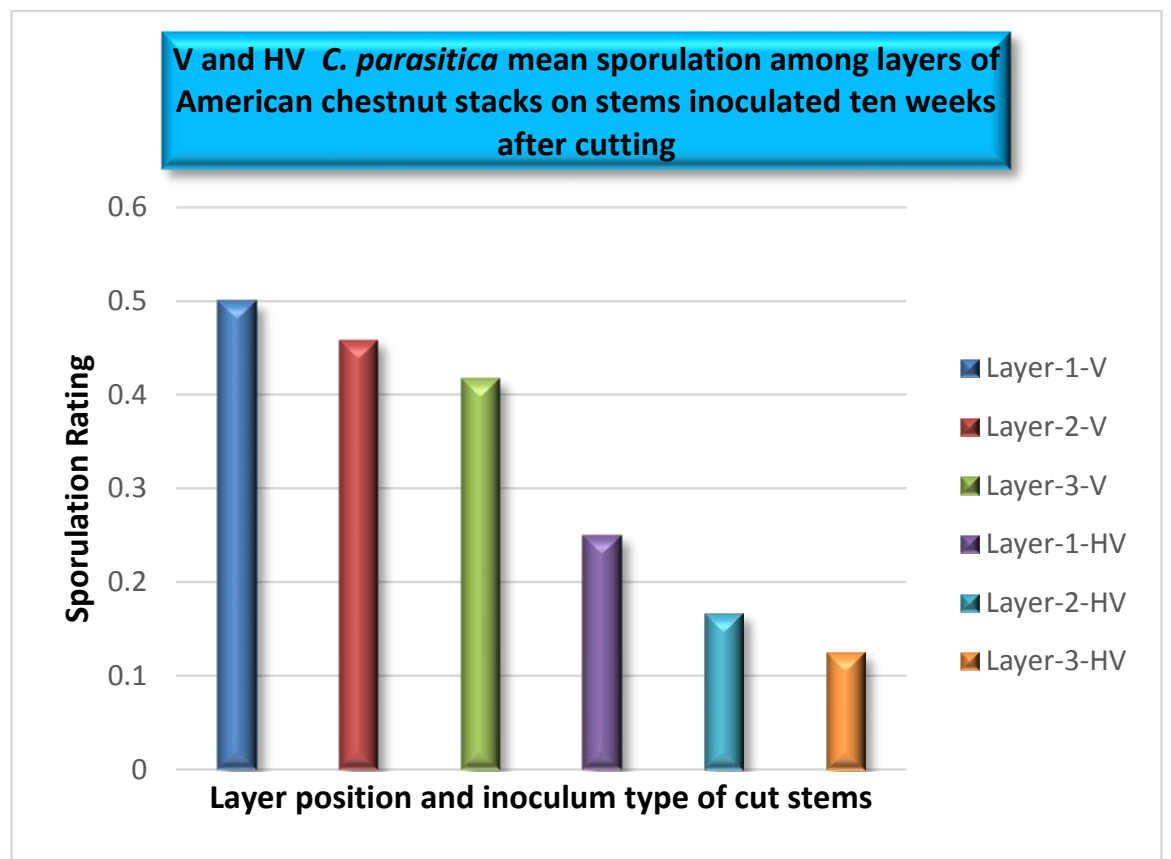

Figure 31: Average sporulation for $\mathrm{V} \mathrm{BRV}-1$ and HV BRHV-1 not significant for $\mathrm{V}$ at $\alpha=0.05(\mathrm{P}>\mathrm{F}=$ 0.9476) or $\mathrm{HV}$ at $(\mathrm{P}>\mathrm{F}=0.6500)$ on American chestnut layers, respectively. L-1 (ground), L-2 (middle) and L-3 (top) for the second inoculation period from August $4^{\text {th }}$ to December $8^{\text {th }} 2011$. 


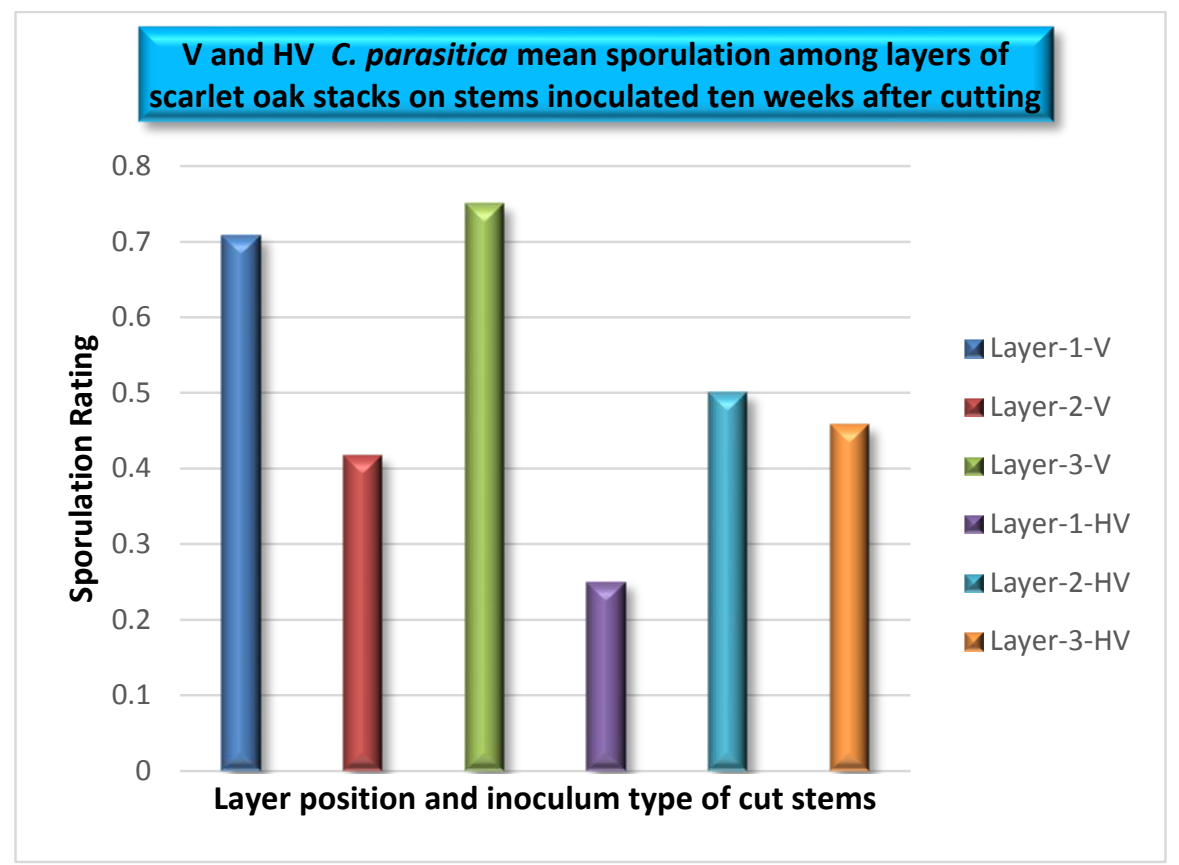

Figure 32: Average sporulation for $V B R V-1$ and $H V B R H V-1$ significant for $V$ at $\alpha=0.05(P>F=0.0169)$ and not for $\mathrm{HV}$ at $(\mathrm{P}>\mathrm{F}=0.4448)$ on scarlet oak layers, respectively. L-1 (ground), L-2 (middle) and L-3 (top) for the second inoculation period from August $4^{\text {th }}$ to December $8^{\text {th }} 2011$.

\section{Third Inoculation Period}

There was sufficient evidence to suggest that the mean difference of V sporulation among the layers within American chestnut stacks were different $(\mathrm{P}>\mathrm{F}=0.0320)$. There were significantly fewer stroma formed on L-3 than the other two layers (Figure 33). However, there was not a significant difference among V sporulation on scarlet oak layers for the third inoculation period $(\mathrm{P}>\mathrm{F}=0.1539)$ (Figure 34).

There was a significant difference for HV sporulation on the layers within a stack of American chestnut $(\mathrm{P}>\mathrm{F}=0.0519)$ (Figure 33), where, L-2 sporulated less than the others. Bark sporulation means of the inoculated scarlet oak stems by HV C. parasitica were not significantly different for any layer $(\mathrm{P}>\mathrm{F}=0.8443)$ (Figure 34$)$. 


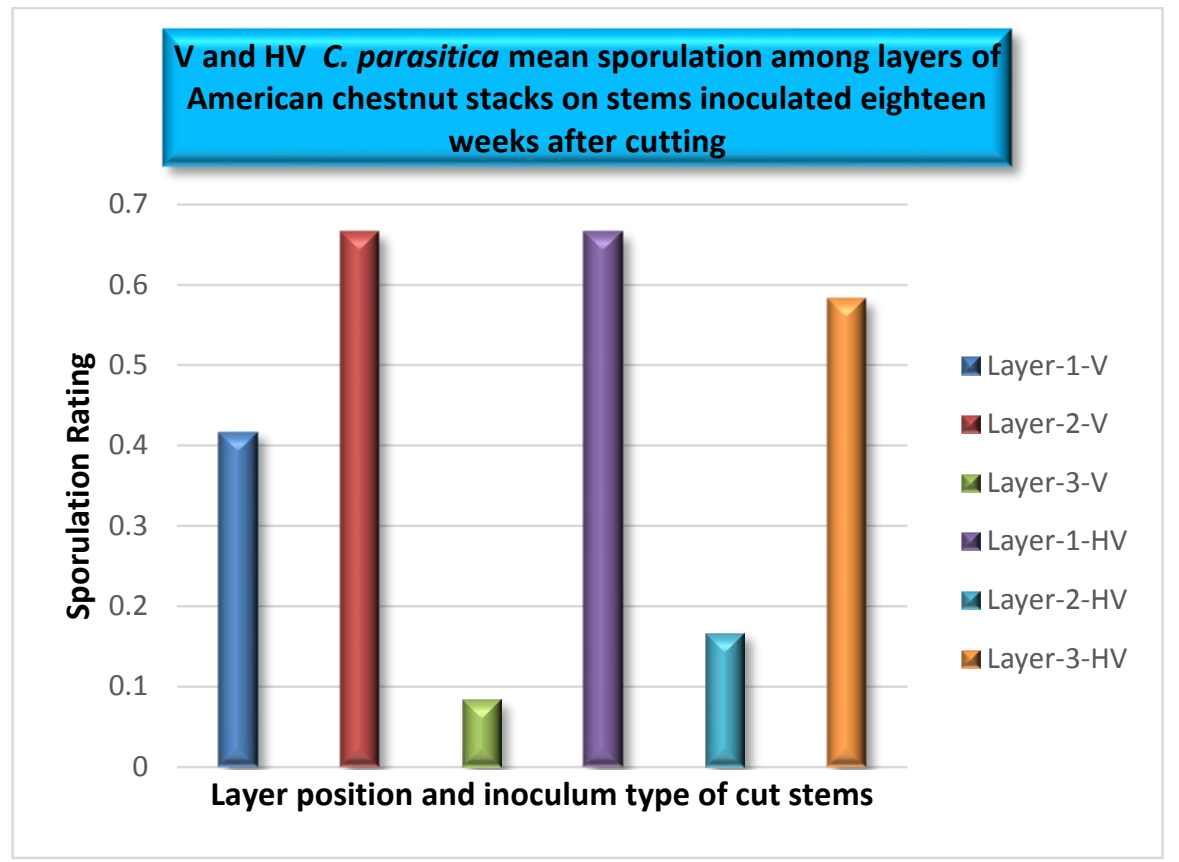

Figure 33: Average sporulation for $\mathrm{V} B R V-1$ and HV BRHV-1 significant for $\mathrm{V}$ at $\alpha=0.05(\mathrm{P}>\mathrm{F}=0.0320)$ and $\mathrm{HV}$ at $(\mathrm{P}>\mathrm{F}=0.0519)$ on American chestnut layers, respectively. L-1 (ground), L-2 (middle) and L-3 (top) for the third inoculation period from October $4^{\text {th }}$ to December $8^{\text {th }} 2011$.

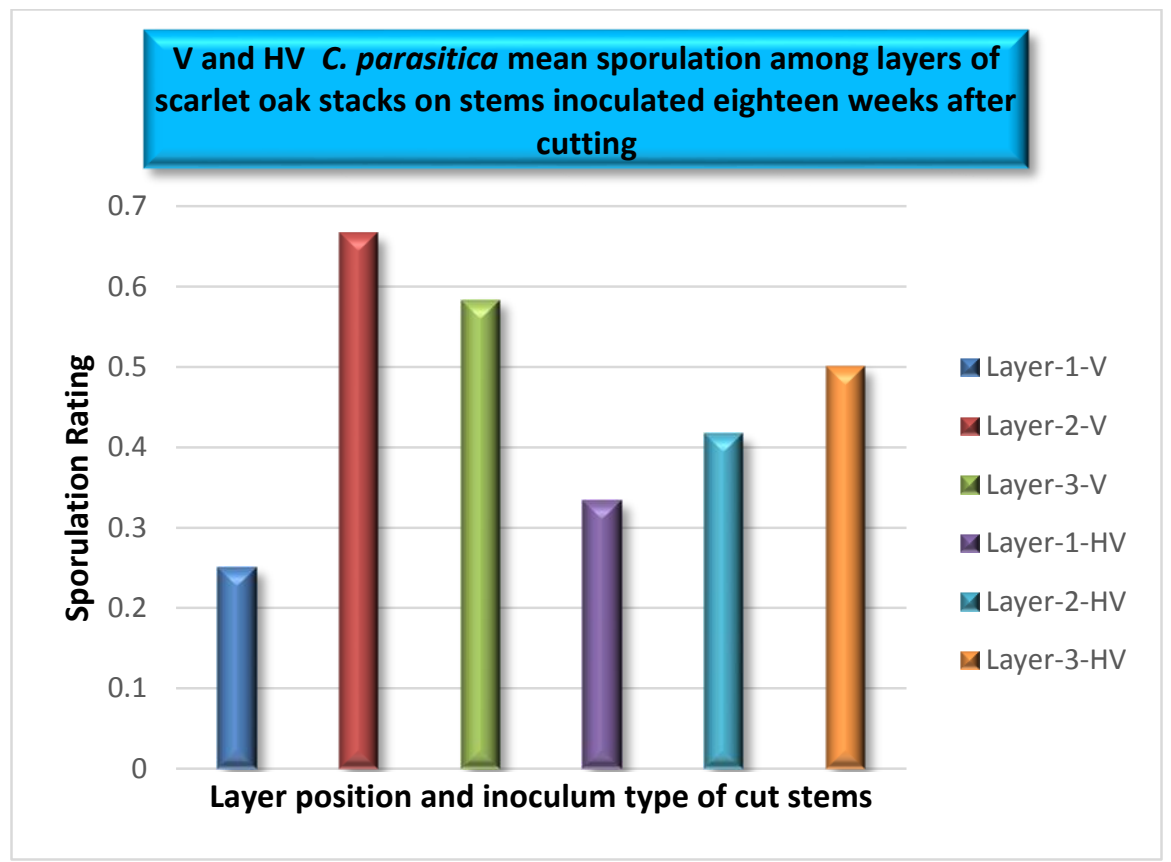

Figure 34: Average sporulation for V BRV-1 and HV BRHV-1 not significant for $\mathrm{V}$ at $\alpha=0.05(\mathrm{P}>\mathrm{F}=$ $0.1539)$ or HV at $(\mathrm{P}>\mathrm{F}=0.8443)$ on scarlet oak layers respectively. L-1 (ground), L-2 (middle) and L-3 (top) for the third inoculation period from October $4^{\text {th }}$ to December $8^{\text {th }} 2011$. 


\section{SECTION 3: Effect of Stack Location on Sporulation}

\section{First Inoculation Period}

In an effort to analyze the effect of location on sporulation a comparison among stacks within a group was made. This test analyzed the total stroma formation that can take place within a mixed stack of chestnut and oak. The ordinal system sporulation ranks for the oak and chestnut stems within a stack receiving the same inoculation type (V, HV and C) were pooled for this analysis. Although groups G-1 through G-5 were spatially distributed, they were all close to one another on a wooded terrace within the study site such that 33 meters was the greatest distance between any stack. G-1 and G-4 were the farthest distances apart. The ANOVA indicated that there was not sufficient evidence to suggest sporulation differences existed within $\mathrm{V}$ stacks $(\mathrm{P}>\mathrm{F}=<0.0623)$. However, the sporulation for stack G-4 was less than the other groups but not significantly so (Figure 35). In contrast, there was a statistically significant difference among the HV groups $(\mathrm{P}>\mathrm{F}=0.0001)$. All comparisons tests indicated that group $\mathrm{G}-4$ had significantly less sporulation than the others (Figure 36).

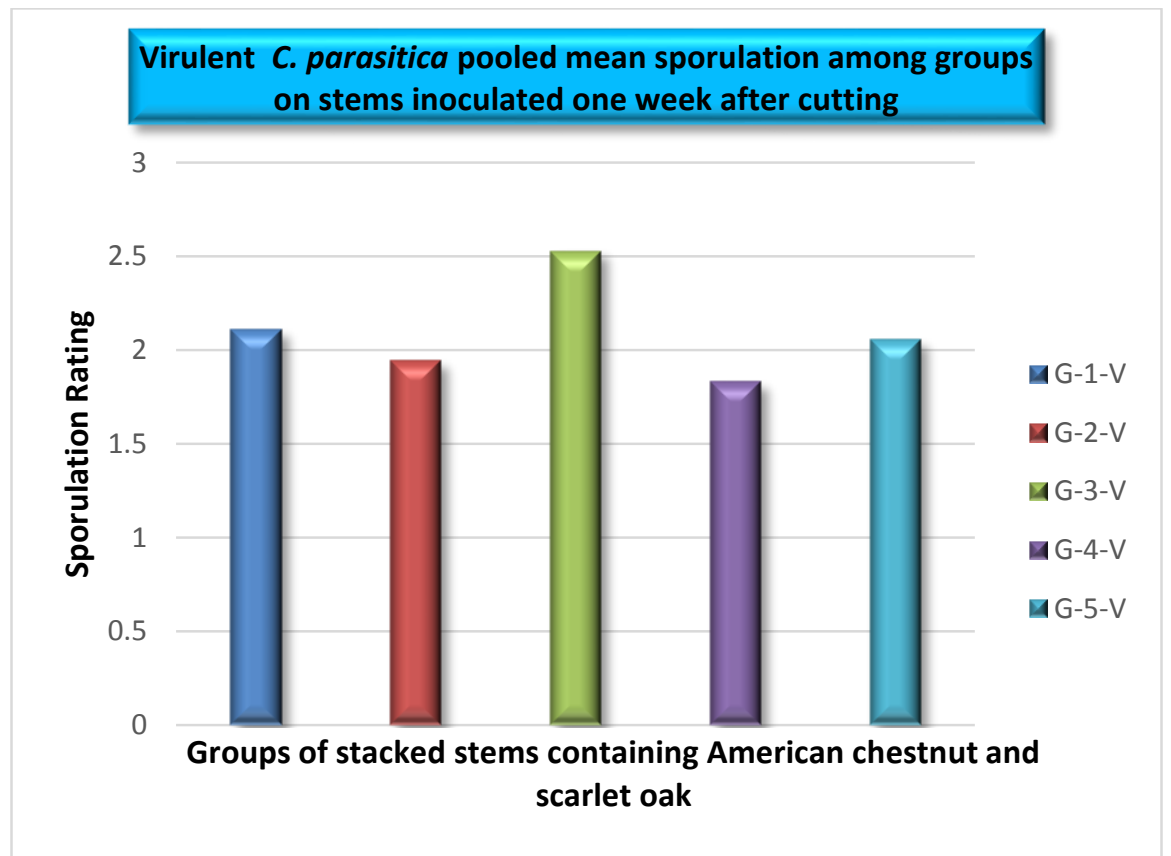

Figure 35: Average sporulation for V BRV-1 not significant at $\alpha=0.05(\mathrm{P}>\mathrm{F}=0.0623)$ for pooled sporulation on American chestnut and scarlet oak within a group for the first inoculation period from May $20^{\text {th }}$ to December $8^{\text {th }} 2011$. 


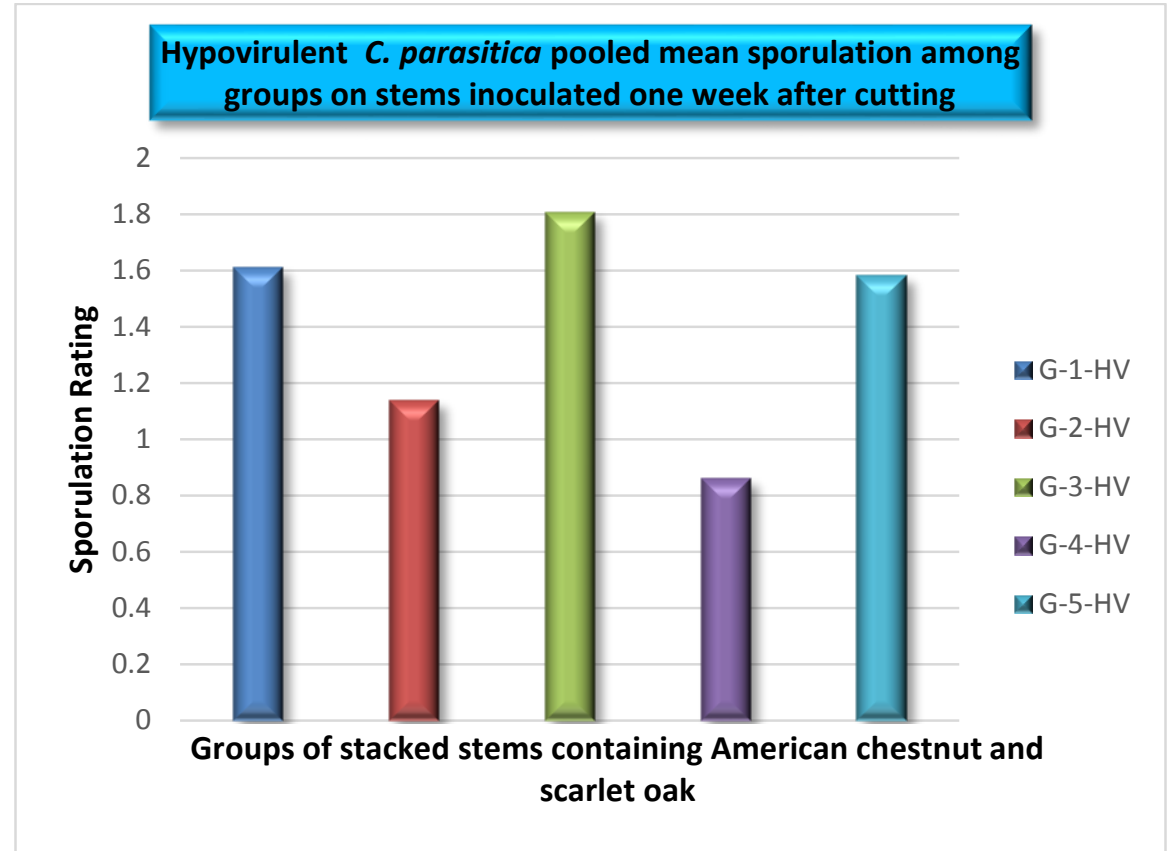

Figure 36: Average sporulation for HV BRHV-1 significant at $\alpha=0.05(\mathrm{P}>\mathrm{F}=0.0001)$ for pooled sporulation on American chestnut and scarlet oak within a group for the first inoculation period from May $20^{\text {th }}$ to December $8^{\text {th }} 2011$.

\section{Second Inoculation Period}

The ANOVA indicated that there was insufficient evidence to suggest sporulation differences existed within $\mathrm{V}(\mathrm{P}>\mathrm{F}=<0.0976)$ (Figure 37) or $\mathrm{HV}$ groups $(\mathrm{P}>\mathrm{F}=0.7104)$ (Figure 38). Though, G-1 sporulated the most for V and HV.

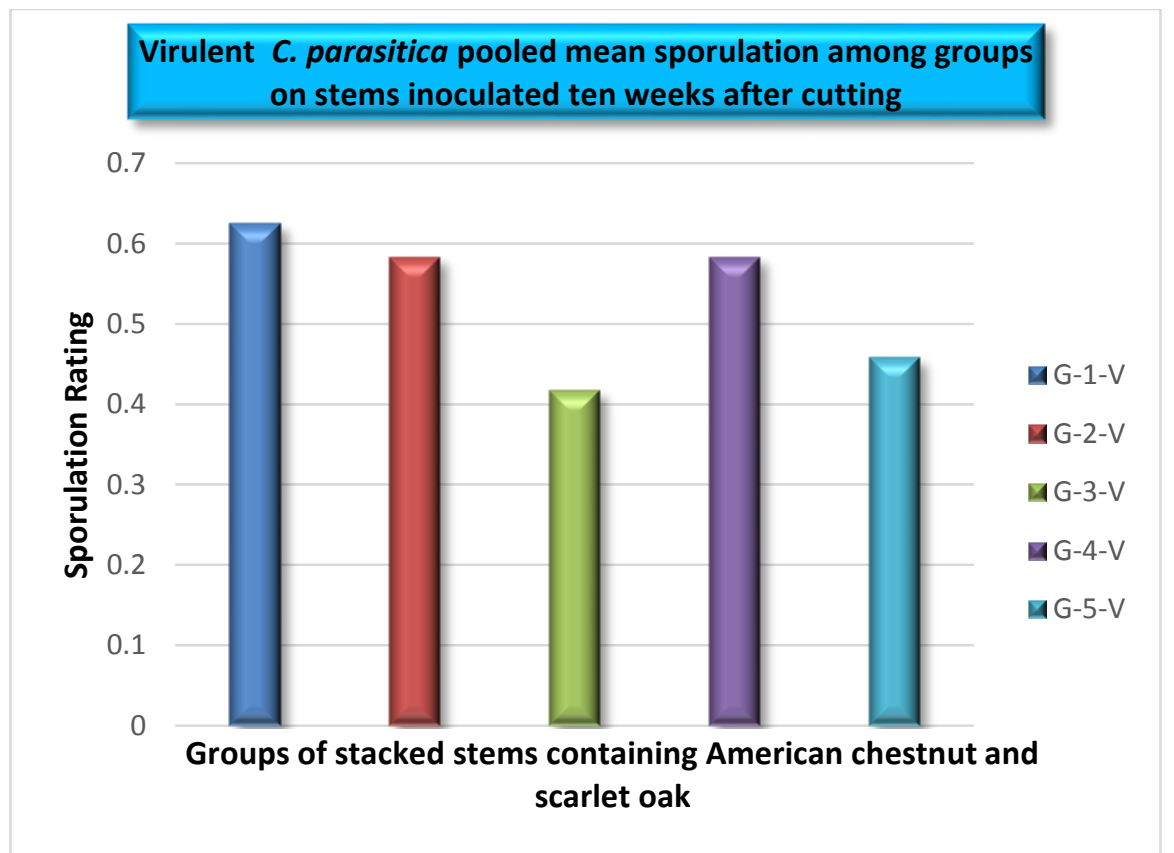

Figure 37: Average sporulation for V BRV-1 not significant at $\alpha=0.05(\mathrm{P}>\mathrm{F}=0.0976)$ for pooled sporulation on American chestnut and scarlet oak within a group for the second inoculation period from August $4^{\text {th }}$ to December $8^{\text {th }} 2011$. 


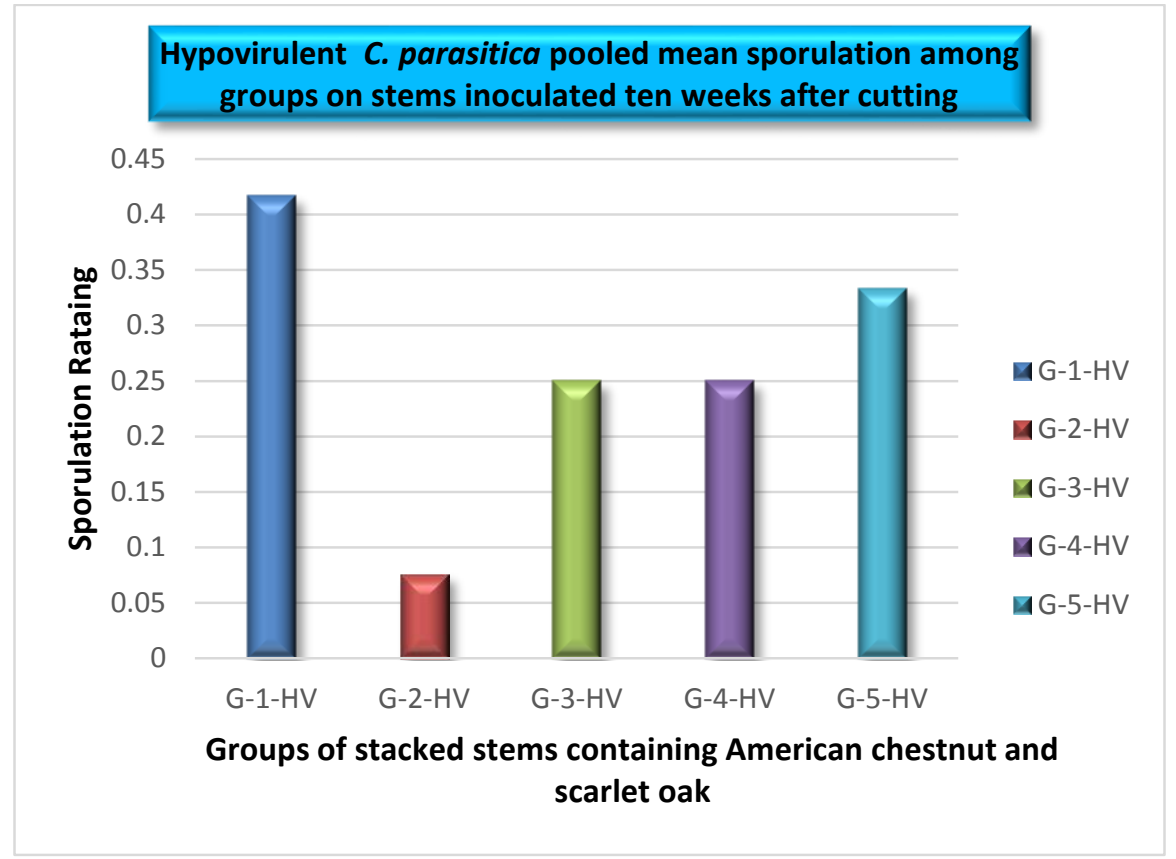

Figure 38: Average sporulation for HV BRHV -1 not significant at $\alpha=0.05(P>F=0.7104)$ for pooled sporulation on American chestnut and scarlet oak within a group for the second inoculation period from August $4^{\text {th }}$ to December $8^{\text {th }} 2011$.

\section{Third Inoculation Period}

The ANOVA indicated that there was insufficient evidence to suggest sporulation differences existed within $\mathrm{V}(\mathrm{P}>\mathrm{F}=<0.2860)$ (Figure 39) or $\mathrm{HV}(\mathrm{P}>\mathrm{F}=0.5420)$ stacks (Figure 40). No trends were recognized during this inoculation period.

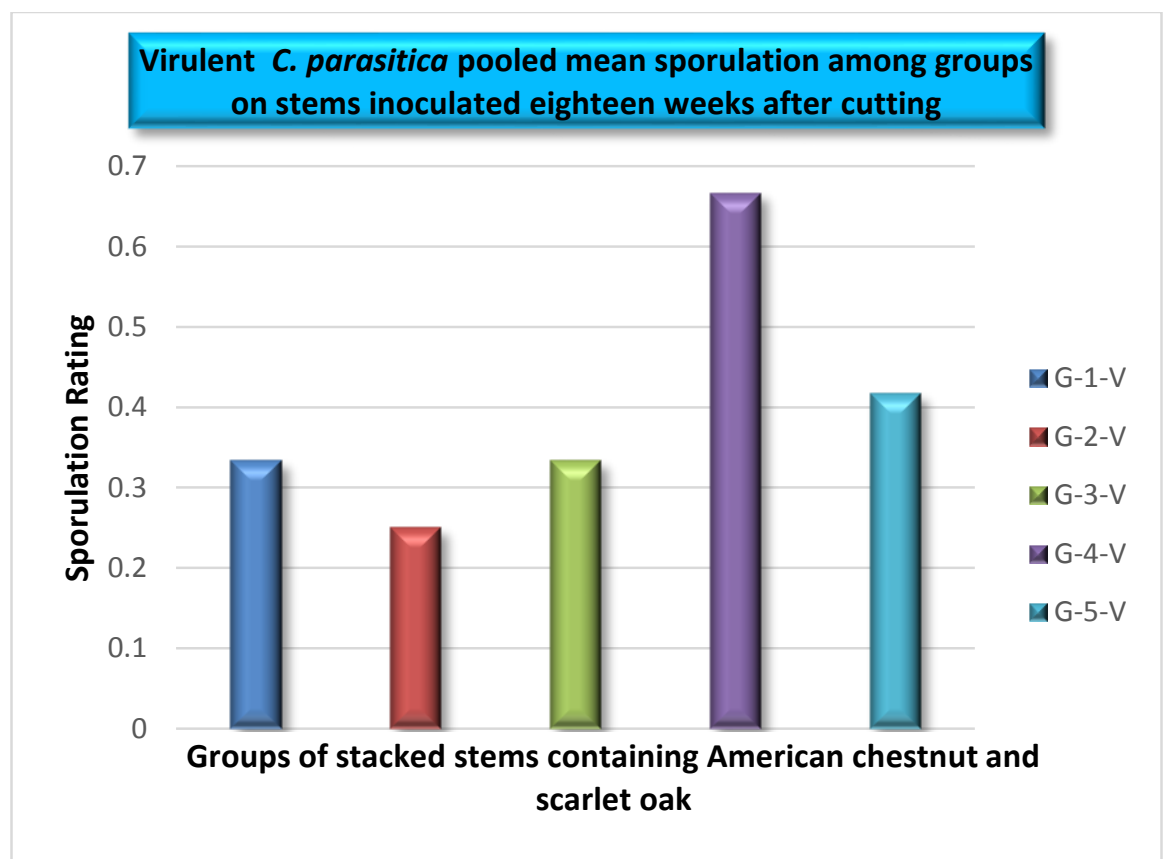

Figure 39: Average sporulation for V BRV-1 not significant at $\alpha=0.05(P>F=0.2860)$ for pooled sporulation on American chestnut and scarlet oak within a group for the third inoculation period from October $4^{\text {th }}$ to December $8^{\text {th }} 2011$. 


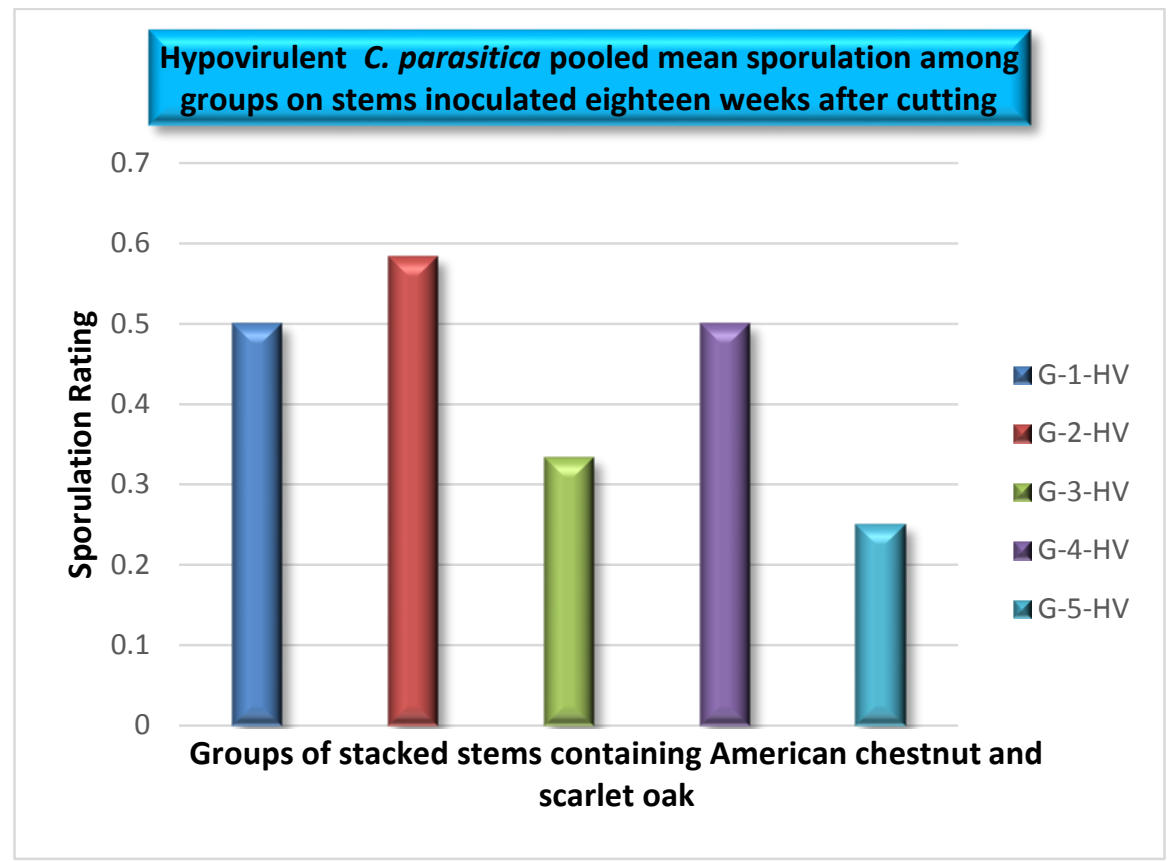

Figure 40: Average sporulation for HV BRHV-1 not significant at $\alpha=0.05(\mathrm{P}>\mathrm{F}=0.5420)$ for pooled sporulation on American chestnut and scarlet oak within a group for the third inoculation period from October $4^{\text {th }}$ to December $8^{\text {th }} 2011$.

\section{CHAPTER 2 SPORULATION SUMMARY: Total Sporulation, Effect of Layer and Location of Stack at the Site}

The results in Tables 5-7 provide a direct comparison of data collected for overall sporulation, the effect of the stack layer in which stems were placed and the effect of the position of the stack at the site for the May, August and October inoculation periods (IP's). This presentation is made because the y-axes differ among the histograms and graphs presented in this chapter. Even though no statistical tests were conducted to compare the inoculation periods, the ability of $C$. parasitica to sporulate diminished with each successive inoculation period (IP). Although there was some variation in sporulation noted among the stems at different layers for the most part there was no consistent trend other than the general reduction in sporulation that occurred as stems aged. Like colonization, the data also support the observation that scarlet oak provided a better substrate for sporulation during IP's 2 and 3 then did American chestnut. Other trends indicated during inoculation periods 2 and 3 reflects higher $\mathrm{HV}$ than $\mathrm{V}$ sporulation 
during both IP's and in a few circumstances more stroma production occurred during

IP-3 than in IP-2 for the three analyses.

TABLE 5: Average C. parasitica total sporulation comparisons for American chestnut and scarlet oak including all treatments and inoculation periods.

\begin{tabular}{|l|l|l|l|l|}
\hline $\begin{array}{l}\text { Total } \\
\text { Sporulation }\end{array}$ & Inoc 1 & Inoc 2 & Inoc 3 \\
\hline & Rating (0-3) & & Rating (0-3) & Rating (0-3) \\
\hline Cd V & 2.53 & 0.45 & $0.33^{\text {d }}$ \\
\hline Qc V & 1.65 & 0.6 & 0.47 \\
\hline Cd HV & 1.9 & $0.16^{\mathrm{e}}$ & 0.43 \\
\hline Qc HV & 0.9 & 0.43 & 0.43 \\
\hline Control $^{\mathbf{b}}$ & 0 & 0 & 0 \\
\hline
\end{tabular}

${ }^{\mathrm{a}} \mathrm{Cd}=$ Castanea dentata $; \mathrm{Qc}=$ Quercus coccinea

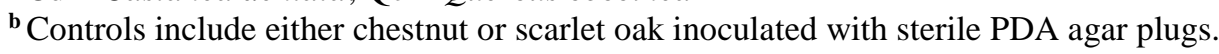

${ }^{\mathrm{c}}$ Sporulation is based using an ordinal rating system, where $0=$ no sporulation, $1=$ minimal sporulation, 2 $=$ medium sporulation, and $3=$ significant sporulation.

d Yellow highlighted text indicates that HV sporulated more than V within the same tree species and layer type.

${ }^{\text {e }}$ Red blocks indicate a region where the subsequent IP's sporulation was greater than the previous IP's.

TABLE 6: Average $C$. parasitica layer effect sporulation comparisons for American chestnut and scarlet oak including all treatments and inoculation periods.

\begin{tabular}{|l|l|l|l|l|}
\hline Layer Sporulation $^{\mathbf{a}}$ & Inoc $\mathbf{1}$ & Inoc 2 & & Inoc 3 \\
\hline Cd-L1-V & Rating (0-3) & Rating (0-3) & Rating (0-3) \\
\hline Cd-L2-V & 2.77 & 0.5 & 0.42 \\
\hline Cd-L3-V & 2.64 & 0.46 & 0.66 \\
\hline Cd-L1-HV & $2.31^{\mathrm{d}}$ & 0.42 & 0.08 \\
\hline Cd-L2-HV & 1.91 & 0.25 & 0.66 \\
\hline Cd-L3-HV & 1.33 & 0.17 & 0.16 \\
\hline Qc-L1-V & 2.44 & 0.13 & 0.58 \\
\hline Qc-L2-V & 2.28 & 0.71 & 0.25 \\
\hline Qc-L3-V & 1.42 & 0.42 & 0.67 \\
\hline Qc-L1-HV & 1.58 & 0.75 & 0.58 \\
\hline Qc-L2-HV & 0.98 & 0.25 & 0.33 \\
\hline Qc-L3-HV & $0.39^{e}$ & 0.5 & 0.42 \\
\hline Control & 1.17 & 0.46 & 0.5 \\
\hline
\end{tabular}

${ }^{\mathrm{a}} \mathrm{Cd}=$ Castanea dentata $; \mathrm{Qc}=$ Quercus coccinea

${ }^{\text {b }}$ Controls include either chestnut or scarlet oak inoculated with sterile PDA agar plugs.

${ }^{\mathrm{c}}$ Sporulation is based using an ordinal rating system, where $0=$ no sporulation, $1=$ minimal sporulation, 2 $=$ medium sporulation, and $3=$ significant sporulation.

d Yellow highlighted text indicates that HV sporulated more than V within the same tree species and layer type.

'Red blocks indicate a region where the subsequent IP's sporulation was greater than the previous IP's. 
TABLE 7: Average C. parasitica location effect sporulation comparisons for American chestnut and scarlet oak including all treatments and inoculation periods.

\begin{tabular}{|l|l|l|l|l|}
\hline Group Sporulation $^{\mathbf{a}}$ & Inoc 1 & Inoc 2 & Inoc 3 \\
\hline & Rating (0-3) $^{\mathbf{c}}$ & Rating (0-3) & Rating (0-3) \\
\hline G1-V & 2.11 & 0.63 & $0.33^{\mathrm{d}}$ \\
\hline G1-HV & 1.61 & $0.42^{\mathrm{e}}$ & 0.5 \\
\hline G2-V & 1.94 & 0.58 & 0.25 \\
\hline G2-HV & 1.14 & 0.08 & 0.58 \\
\hline G3-V & 2.53 & 0.42 & 0.33 \\
\hline G3-HV & 1.81 & 0.25 & 0.33 \\
\hline G4-V & 1.83 & 0.58 & 0.67 \\
\hline G4-HV & 0.86 & 0.25 & 0.5 \\
\hline G5-V & 2.06 & 0.46 & 0.42 \\
\hline G5-HV & 1.58 & 0.33 & 0.25 \\
\hline Control & 0 & 0 & 0 \\
\hline
\end{tabular}

${ }^{\text {a }}$ Represents total combined Group sporulation within full, mixed stem stacks of $C$. dentata and $Q$. coccinea

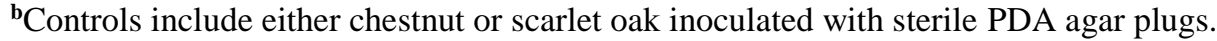

${ }^{\mathrm{c}}$ Sporulation is based using an ordinal rating system, where $0=$ no sporulation, $1=$ minimal sporulation, 2 $=$ medium sporulation, and $3=$ significant sporulation.

${ }^{\mathbf{d}}$ Yellow highlighted text indicates that HV sporulated more than $\mathrm{V}$ within the same tree species and layer type.

'Red blocks indicate a region where the subsequent IP's sporulation was greater than the previous IP's.

\section{CHAPTER 3: Fungi Associated with Inoculated Stems}

Bark plug inoculations made from inoculated stems in the perceived area of infection provided a picture of the fungi that colonized the dead stems following inoculation periods by the $\mathrm{V}$ or $\mathrm{HV}$ strains. Colonies of fungi that grew from the plugs were identified for each of the inoculation periods (Appendix Figures 63-73).

\section{SECTION 1: Castanea dentata V Inoculation}

Figures 41-43 illustrate the various organisms that were recovered from inoculated chestnut bark during each inoculation period. The V C. parasitica used for the May inoculations was recovered from over $80 \%$ of the bark plugs cultured over the duration of the first IP. By the second IP, the recovery of this isolate had decreased to $40 \%$ and by the third inoculation period the $\mathrm{V}$ isolate was only recovered from $12 \%$ of the samples. As isolation of the $\mathrm{V}$ isolate decreased there was a significant increase in 
the recovery of other fungi. Most notable was the increase in the recovery of

Trichoderma spp. By the third IP, it was isolated from $71 \%$ of the bark plugs. Also, notable is the recovery of the $\mathrm{HV} C$. parasitica isolate from each inoculation period ranging from $4 \%, 4 \%$ to $2 \%$ from IP- 1 through 3 (Table 8 ).

\section{FIRST INOCULATION PERIOD}

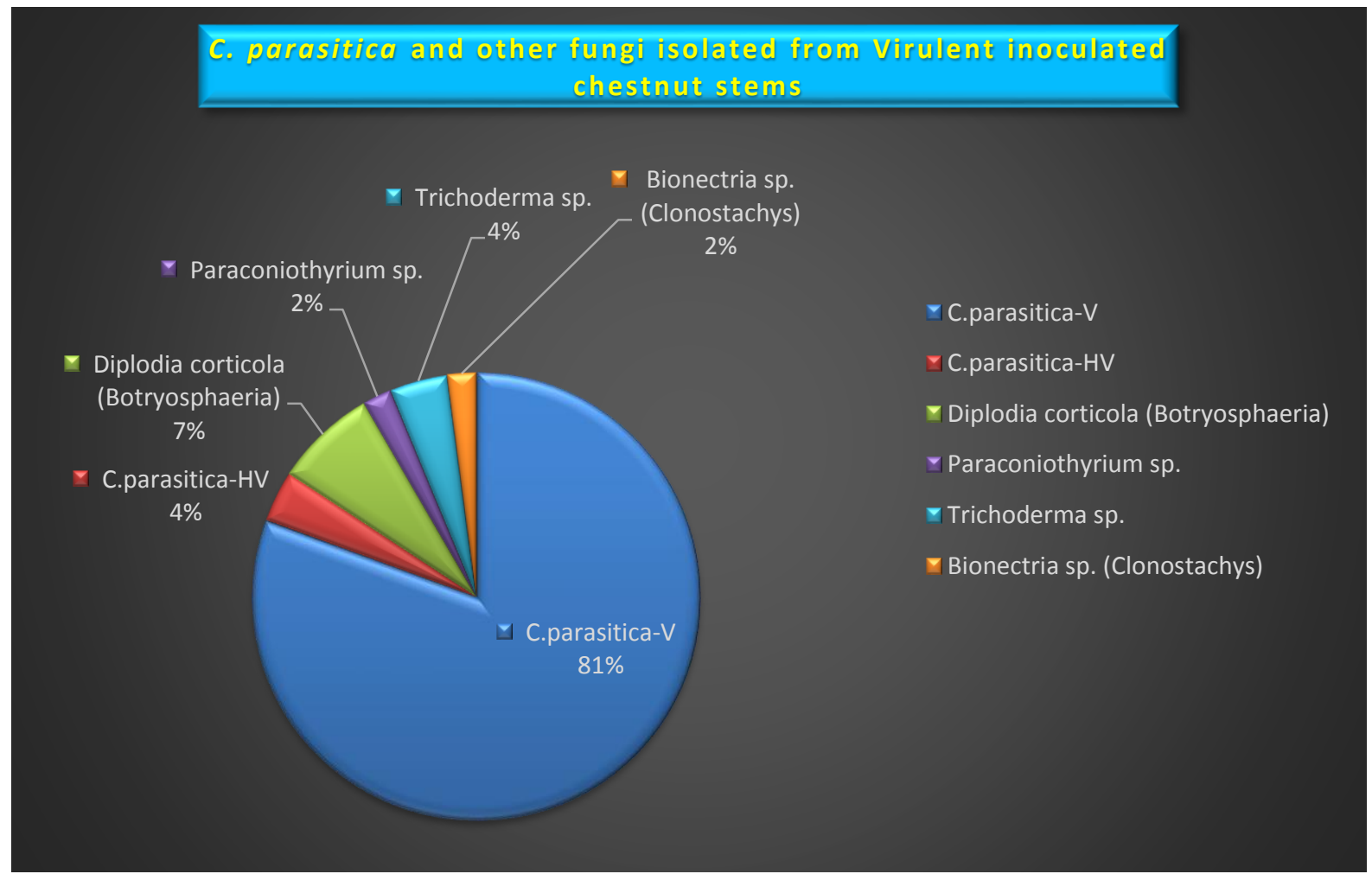

Figure 41: Compilation of fungi recovered from first inoculation period stem infections and sampled at monthly intervals for the duration of the experiment for the May $20^{\text {th }}$ to December $8^{\text {th }}, 2011$ inoculation period. 


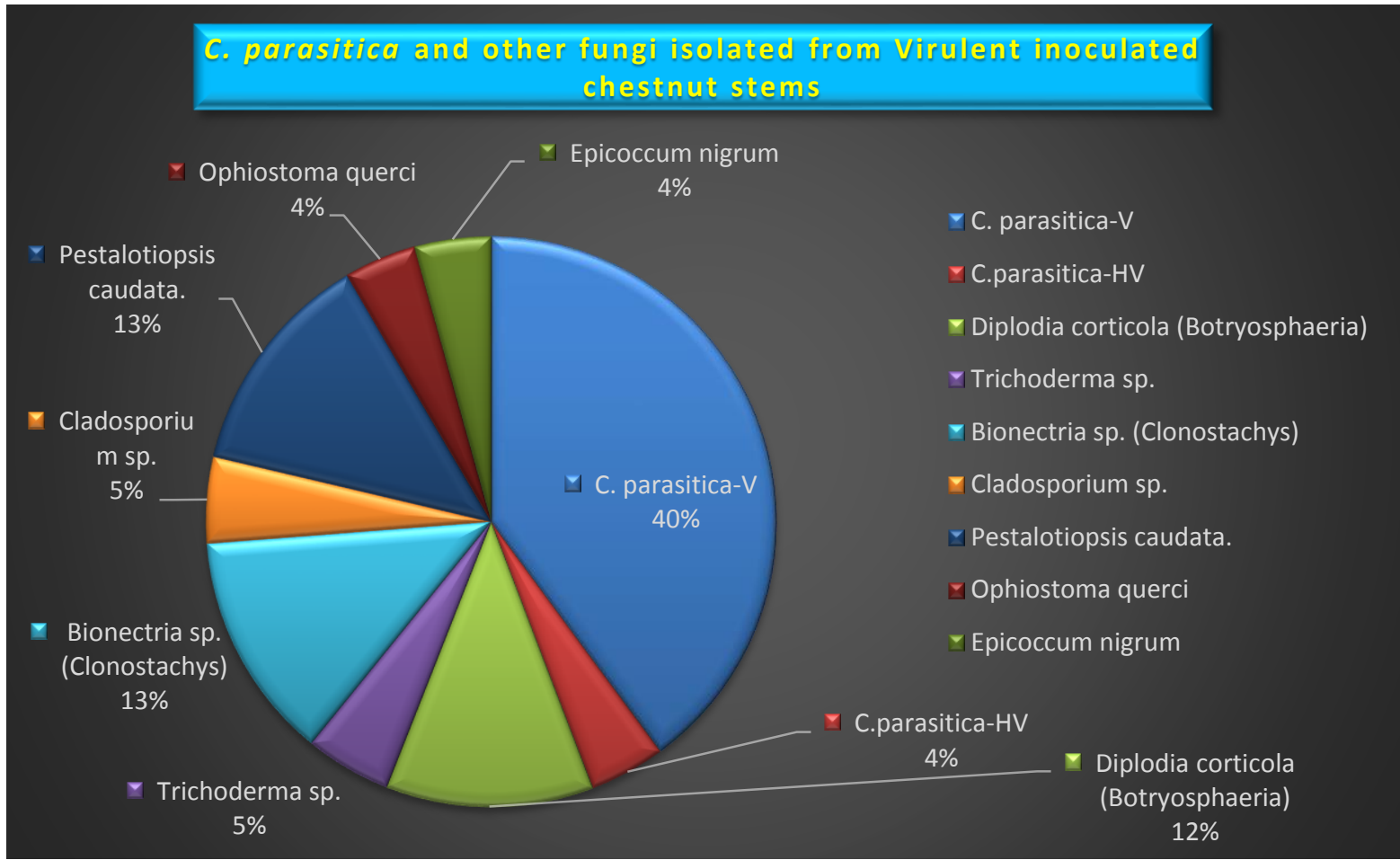

Figure 42: Compilation of fungi recovered from second inoculation period stem infections and sampled at monthly intervals for the duration of the experiment for the August $4^{\text {th }}$ to December $8^{\text {th }}, 2011$ inoculation period.

\section{THIRD INOCULATION PERIOD}

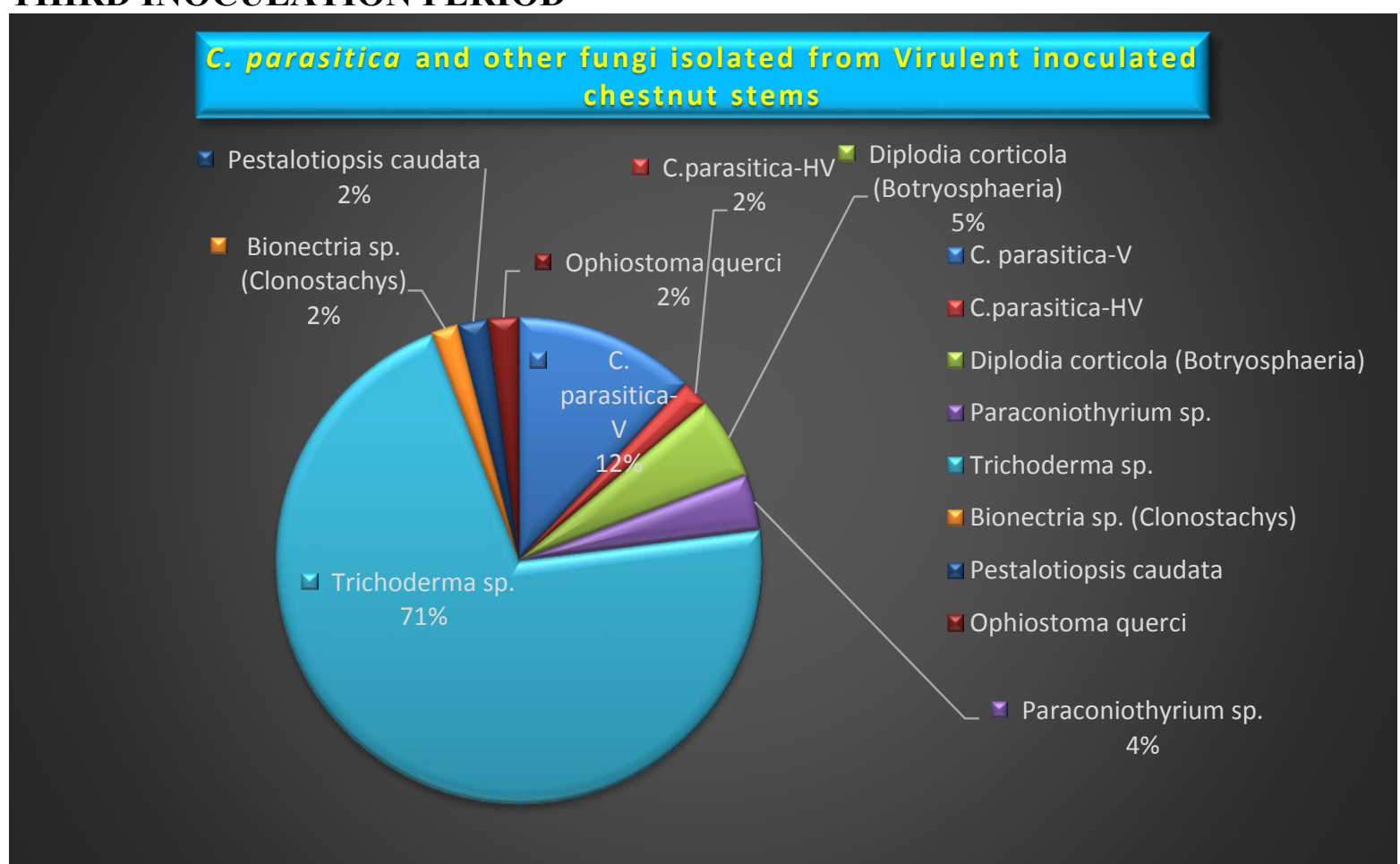

Figure 43: Compilation of fungi recovered from third inoculation period stem infections and sampled at monthly intervals for the duration of the experiment for the October $4^{\text {th }}$ to December $8^{\text {th }}, 2011$ inoculation period. 
TABLE 8: Inoculation period comparison of fungi recovered from stem infections and sampled at monthly intervals for the duration of the experiment for $\mathrm{V}$ inoculated Castanea dentata.

\begin{tabular}{|c|c|c|c|c|}
\hline $\begin{array}{l}\text { Stem/Inoc } \\
\text { Type }\end{array}$ & Fungal Type $^{\mathrm{a}}$ & Inoc 1 & Inoc2 & Inoc 3 \\
\hline \multirow[t]{15}{*}{ C. dentata-V } & & $(\%)$ & $(\%)$ & $(\%)$ \\
\hline & C. parasitica-V & $81^{\mathrm{a}}$ & 40 & 12 \\
\hline & C. parasitica-HV & 4 & 4 & 2 \\
\hline & $\begin{array}{l}\text { Diplodia corticola } \\
\text { (Botrysphearia spp.) }\end{array}$ & $7^{b}$ & 12 & 5 \\
\hline & Paraconiothyrium spp. & 2 & 13 & 4 \\
\hline & Trichoderma spp. & $4^{c}$ & 5 & 71 \\
\hline & $\begin{array}{l}\text { Bionectria spp. } \\
\text { (Clonostachys spp.) }\end{array}$ & 2 & 13 & 2 \\
\hline & Mucor fragilis & $\mathbf{0}$ & $\mathbf{0}$ & $\mathbf{0}$ \\
\hline & Cladosporium spp. & $\mathbf{0}$ & 5 & $\mathbf{0}$ \\
\hline & Pestalotiopsis caudata & $\mathbf{0}$ & 13 & 2 \\
\hline & Xylaria spp. & $\mathbf{0}$ & $\mathbf{0}$ & $\mathbf{0}$ \\
\hline & Epicoccum nigrum & $\mathbf{0}$ & 4 & $\mathbf{0}$ \\
\hline & Ophiostoma querci & $\mathbf{0}$ & 4 & 2 \\
\hline & Phomopsis spp. & $\mathbf{0}$ & $\mathbf{0}$ & $\mathbf{0}$ \\
\hline & Umbelopsis isabellina & $\mathbf{0}$ & $\mathbf{0}$ & $\mathbf{0}$ \\
\hline
\end{tabular}

a Green highlighted blocks indicate the most recovered isolate percentage for each inoculation period.

b Pink highlighted blocks indicate the $2^{\text {nd }}$ most recovered isolate percentage for each inoculation period.

c Aqua highlighted blocks indicate the $3^{\text {rd }}$ most recovered isolate percentage for each inoculation period.

\section{SECTION 2: Castanea dentata HV Inoculation}

When the HV C. parasitica isolate was used to inoculate the chestnut stems the fungi recovered were similar to those recovered from the $\mathrm{V}$ inoculated stems (Figures 4446). Again, with each IP fewer samples yielded the HV isolates. The May inoculations HV isolate was recovered from $60 \%$ of the bark plugs cultured from the first sample period. By the second IP the recovery of this isolate had decreased to $29 \%$ and by IP-3, the $\mathrm{HV}$ isolate was only recovered from $13 \%$ of the samples. As isolation of the $\mathrm{HV}$ isolate decreased there was a significant increase in the recovery of other fungi. Most notable was the increase in the recovery of Trichoderma spp., which by December was isolated from $77 \%$ of the bark plugs. The $\mathrm{V}$ isolate also was recovered from $\mathrm{HV}$ inoculated stems across all three inoculation periods ranging from IP- 1 through IP- 3 at, $20 \%, 9 \%$ to $2 \%$ (Table 9 ). 
FIRST INOCULATION PERIOD

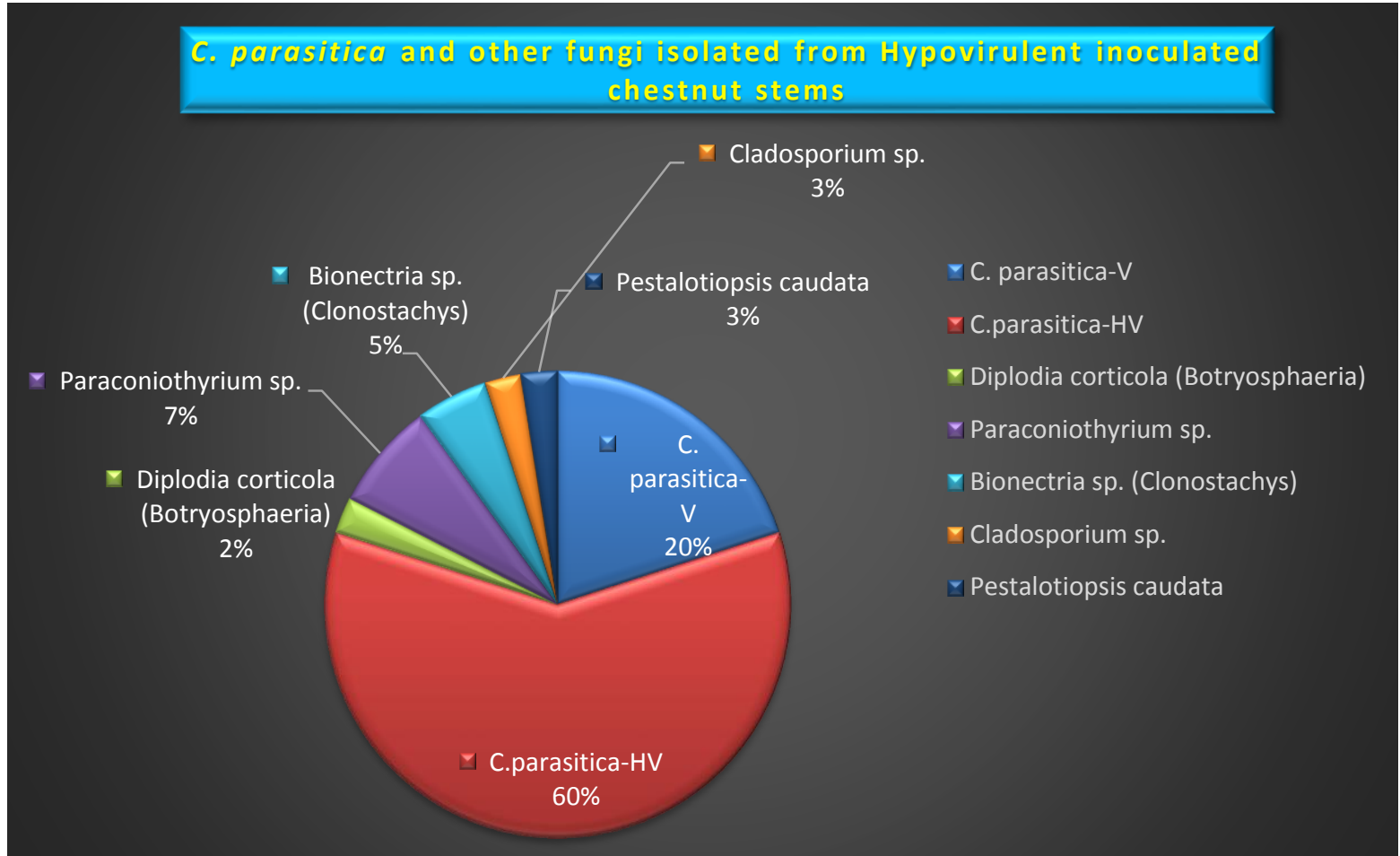

Figure 44: Compilation of fungi recovered from first inoculation period stem infections and sampled at monthly intervals for the duration of the experiment for the May $20^{\text {th }}$ to December $8^{\text {th }}, 2011$ inoculation period.

\section{SECOND INOCULATION PERIOD}

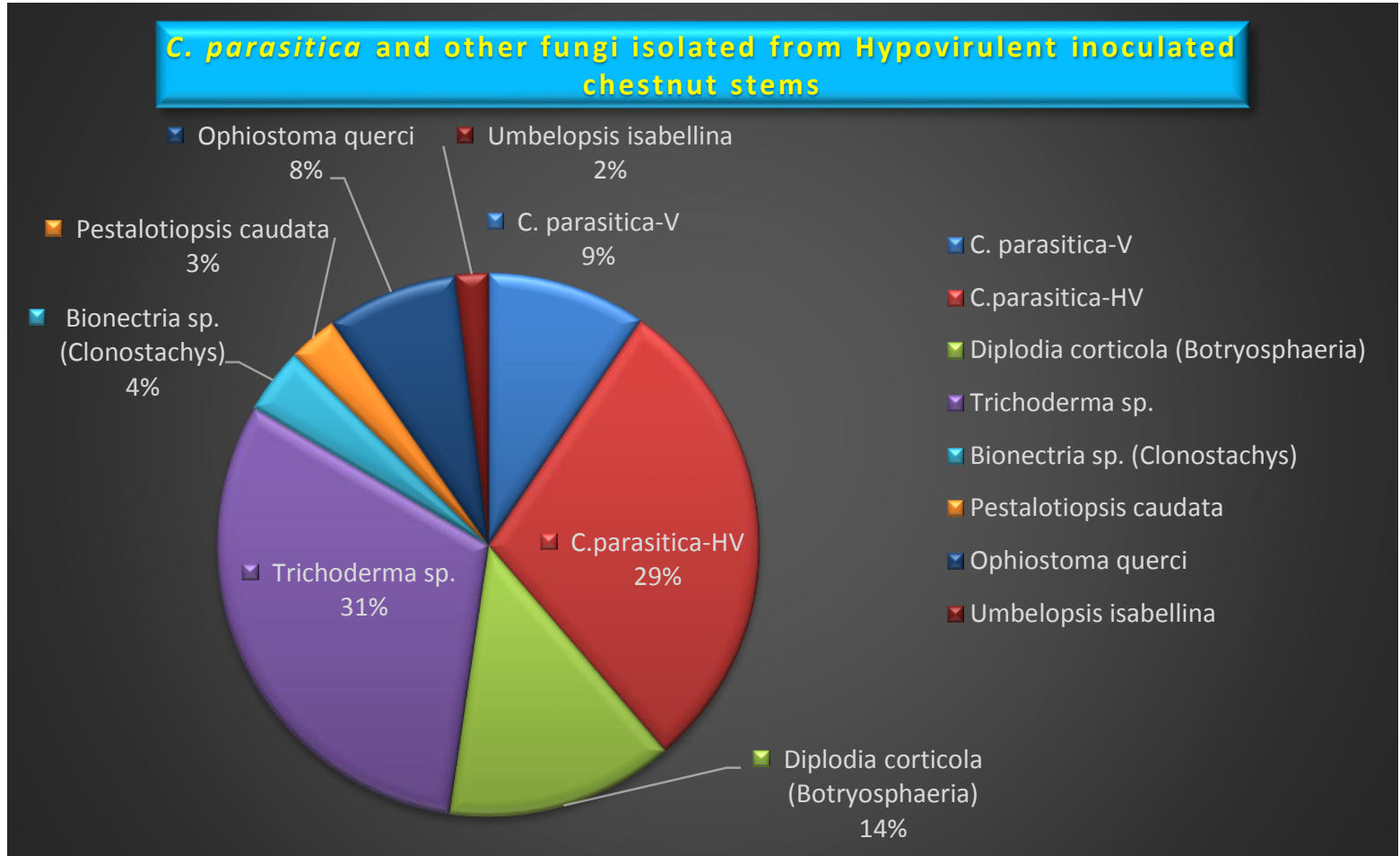

Figure 45: Compilation of fungi recovered from second inoculation period stem infections and sampled at monthly intervals for the duration of the experiment for the August $4^{\text {th }}$ to December $8^{\text {th }}, 2011$ inoculation period 


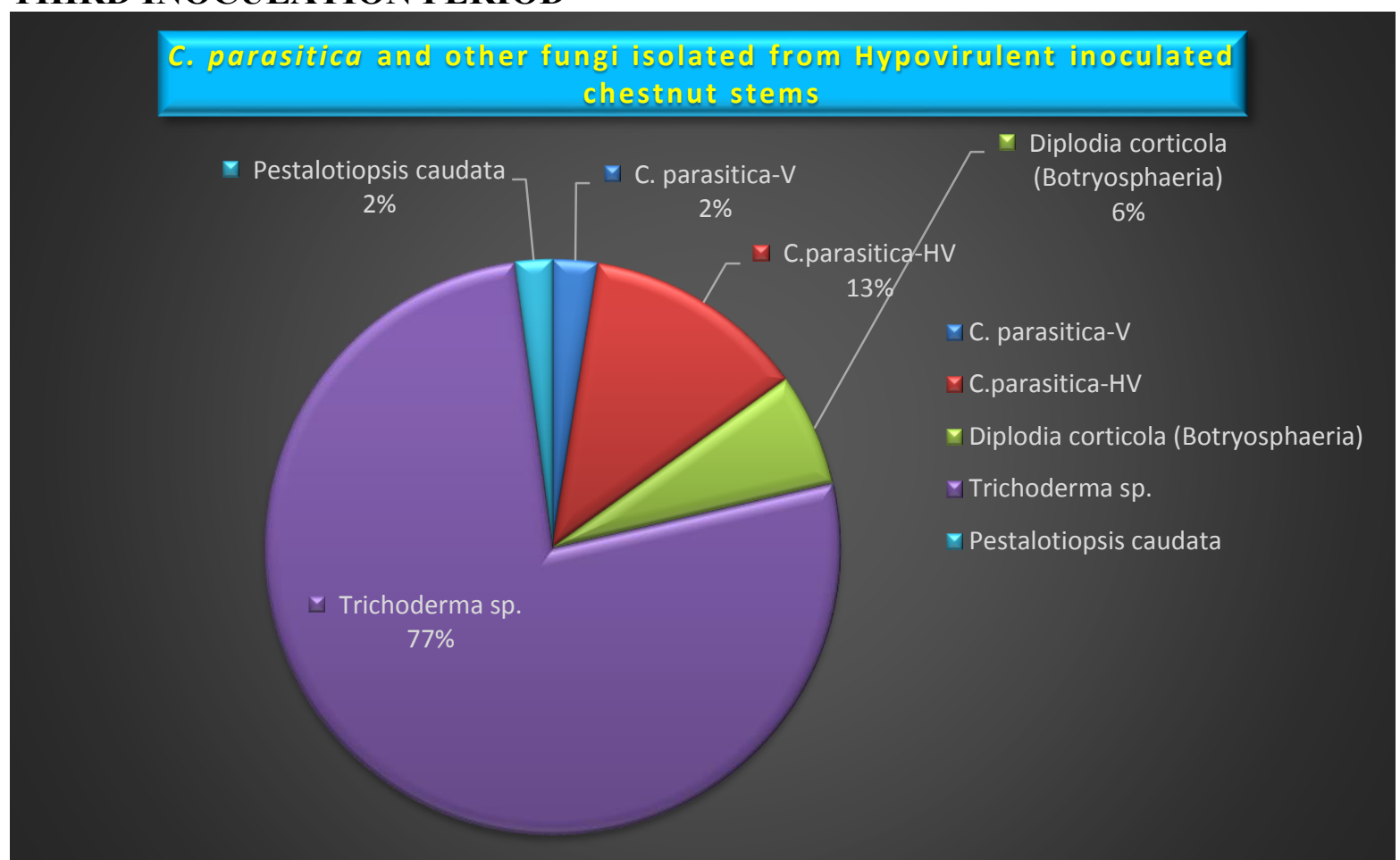

Figure 46: Compilation of fungi recovered from third inoculation period stem infections and sampled at monthly intervals for the duration of the experiment for the October $4^{\text {th }}$ to December $8^{\text {th }}, 2011$ inoculation period.

TABLE 9: Inoculation period comparison of fungi recovered from stem infections and sampled at monthly intervals for the duration of the experiment for HV inoculated Castanea dentata.

\begin{tabular}{|l|l|l|l|l|l|}
\hline $\begin{array}{l}\text { Stem/Inoc } \\
\text { Type }\end{array}$ & Fungal Type $^{\text {a }}$ & Inoc 1 & Inoc2 & Inoc 3 \\
\hline C. dentata-HV & & $(\%)$ & $(\%)$ & $(\%)$ \\
\hline & C. parasitica- $\boldsymbol{V}$ & $20^{\mathrm{b}}$ & 9 & 2 \\
& C. parasitica-HV & $60^{\mathrm{a}}$ & 29 & 13 \\
\hline & $\begin{array}{l}\text { Diplodia corticola } \\
\text { (Botrysphearia spp. } \text { ) }\end{array}$ & 2 & 14 & 6 \\
\hline & Paraconiothyrium spp. & $7^{\mathrm{c}}$ & 0 & 0 \\
\hline & Trichoderma spp. & 0 & 31 & 77 \\
\hline & $\begin{array}{l}\text { Bionectria spp. } \\
\text { (Clonostachys spp.) }\end{array}$ & 5 & 4 & 0 \\
\hline & Mucor fragilis & 0 & 3 & 0 & 0 \\
\hline & Cladosporium spp. & 3 & 0 & 0 \\
\hline & Pestalotiopsis caudata & 0 & 3 & 2 \\
\hline & Xylaria spp. & 0 & 0 & 0 \\
\hline & Epicoccum nigrum & 0 & 0 & 0 \\
\hline & Ophiostoma querci & 0 & 8 & 0 \\
\hline & Phomopsis spp. & 0 & 0 & 0 \\
\hline & Umbelopsis isabellina & 2 & 0 \\
\hline
\end{tabular}

a Green highlighted blocks indicate the most recovered isolate percentage for each inoculation period.

b Pink highlighted blocks indicate the $2^{\text {nd }}$ most recovered isolate percentage for each inoculation period.

c Aqua highlighted blocks indicate the $3^{\text {rd }}$ most recovered isolate percentage for each inoculation period. 


\section{SECTION 3: Castanea dentata (Water Agar) Control Inoculation}

When water agar was used as a control inoculum, the fungi other than $C$.

parasitica, cultured from were similar to those recovered from the $\mathrm{V}$ and $\mathrm{HV}$ inoculated stems (Figures 47-49). Even though the $\mathrm{V}$ isolate was not used for inoculation it was recovered from the first (9\%) and second (6\%) inoculation periods. No HV isolates were recovered. The most prominent species recovered was Trichoderma spp. increasing from $21 \%, 58 \%$ to $73 \%$ from IP-1 through IP-3. Pestalotiopsis caudata was the second most prominent species for the first (16\%) and second (15\%) period but Diplodia corticola (17\%) and Ophiostoma querci $(10 \%)$ were the only other recovered fungi except

Trichoderma spp. by the time of the third IP (Table 10).

\section{FIRST INOCULATION PERIOD}

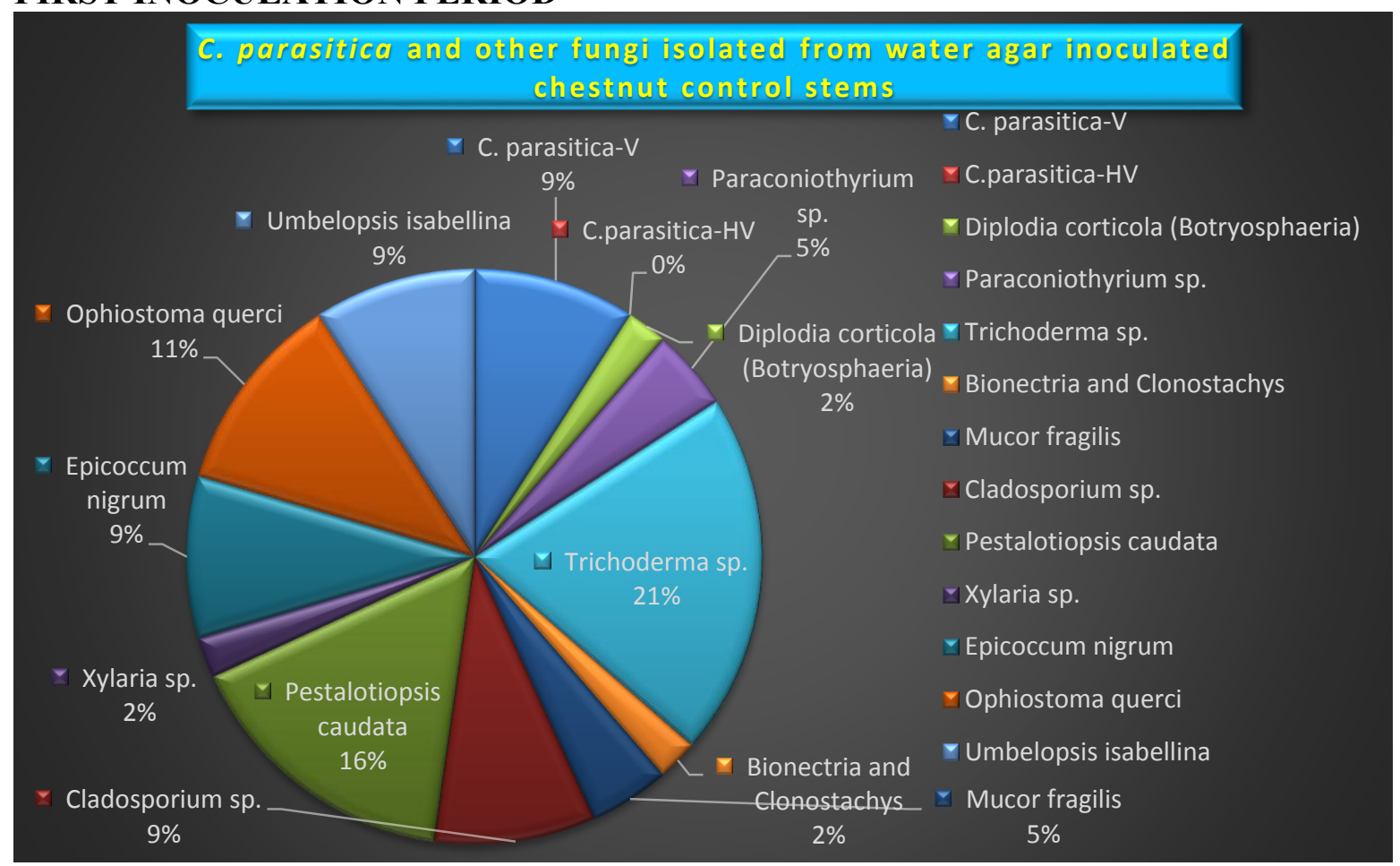

Figure 47: Compilation of fungi recovered from first inoculation period stems and sampled at monthly intervals for the duration of the experiment for the May $20^{\text {th }}$ to December $8^{\text {th }}, 2011$ inoculation period. 


\section{SECOND INOCULATION PERIOD}

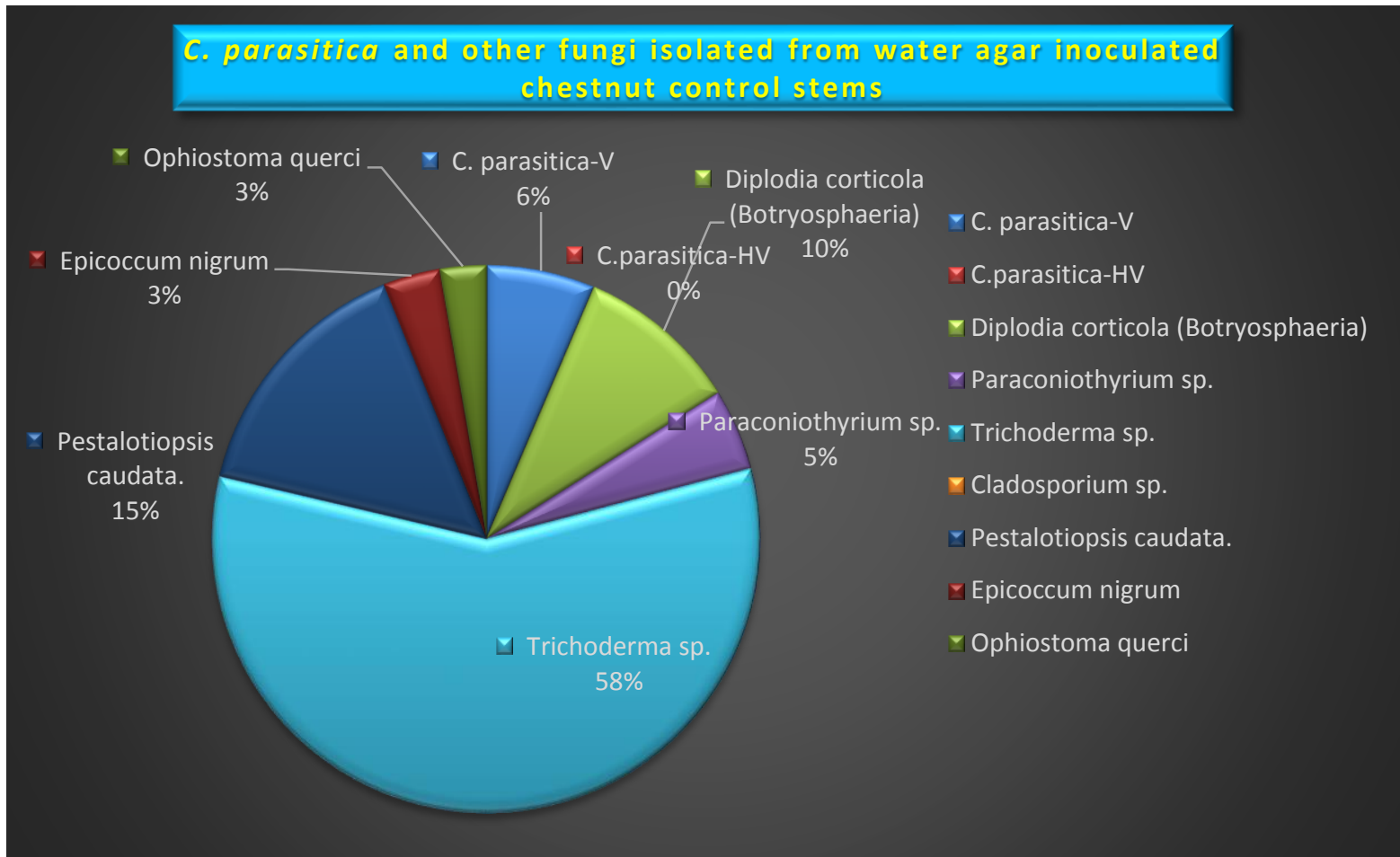

Figure 48: Compilation of fungi recovered from second inoculation period stems and sampled at monthly intervals for the duration of the experiment for the August $4^{\text {th }}$ to December $8^{\text {th }}, 2011$ inoculation period.

\section{THIRD INOCULATION PERIOD}

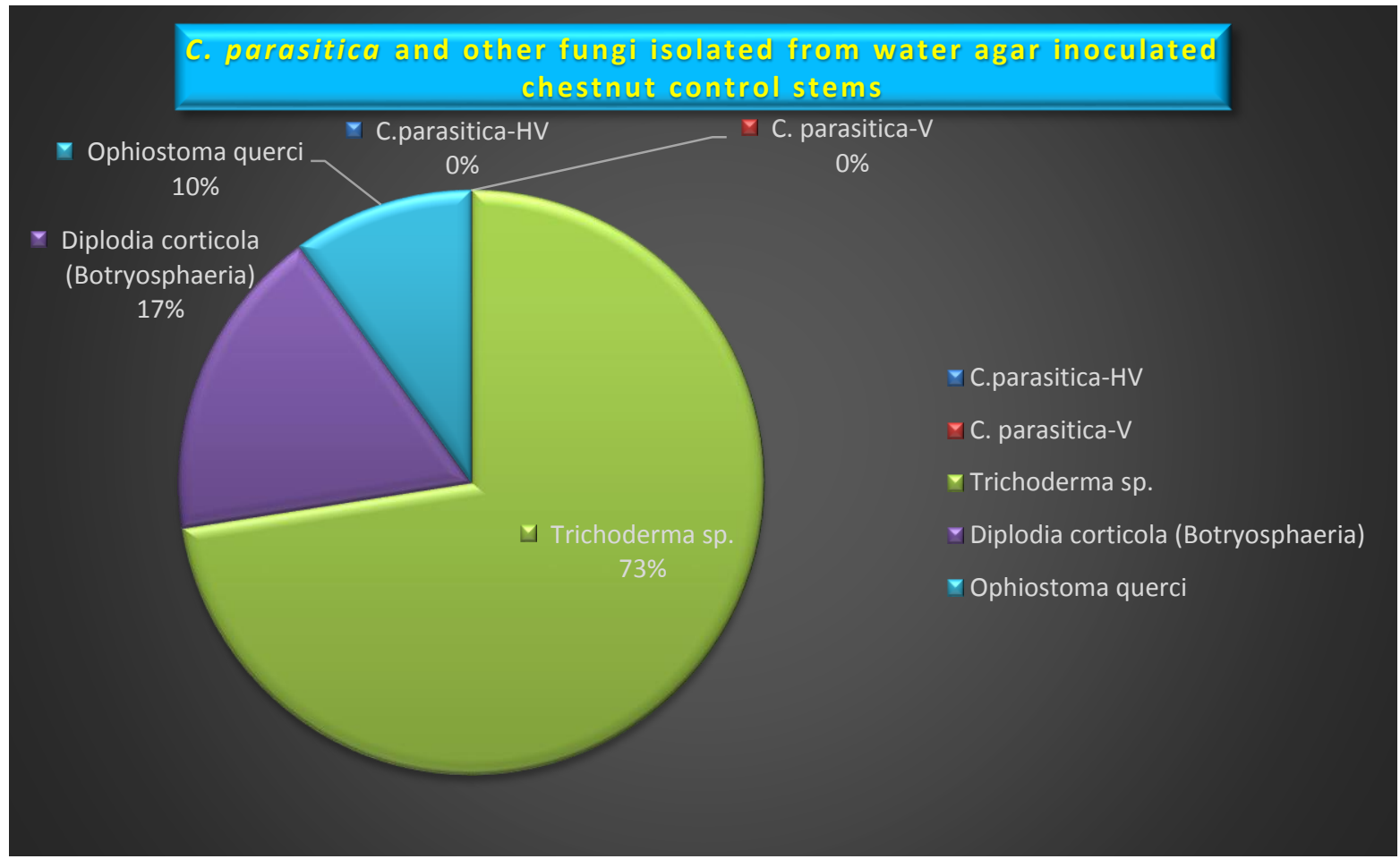

Figure 49: Compilation of fungi recovered from third inoculation period stems and sampled at monthly intervals for the duration of the experiment for the October $4^{\text {th }}$ to December $8^{\text {th }}, 2011$ inoculation period. 
TABLE 12: Inoculation period comparison of fungi recovered from stems and sampled at monthly intervals for the duration of the experiment for Water Ager inoculated Castanea dentata.

\begin{tabular}{|l|l|l|l|l|l|}
\hline $\begin{array}{l}\text { Stem/Inoc } \\
\text { Type }\end{array}$ & Fungal Type $^{\text {a }}$ & Inoc 1 & Inoc 2 & Inoc 3 \\
\hline $\begin{array}{l}\text { C. dentata- } \\
\text { Control }\end{array}$ & & $(\%)$ & $(\%)$ & $(\%)$ \\
\hline & C. parasitica- $\boldsymbol{V}$ & 9 & 6 & 0 \\
\hline & $\begin{array}{l}\text { C. parasitica-HV } \\
\text { Diplodia corticola } \\
\text { (Botrysphearia spp.) }\end{array}$ & 0 & 0 & 0 \\
& Paraconiothyrium spp. & 5 & 10 & 17 \\
& Trichoderma spp. & 21 & 5 & 0 \\
\hline & $\begin{array}{l}\text { Bionectria spp. } \\
\text { (Clonostachys spp.) }\end{array}$ & 2 & 58 & 73 \\
& Mucor fragilis & 5 & 0 & 0 \\
\hline & Cladosporium spp. & 9 & 0 & 0 \\
\hline & Pestalotiopsis caudata & 16 & 0 & 0 \\
\hline & Xylaria spp. & 2 & 15 & 0 \\
\hline & Epicoccum nigrum & 9 & 0 & 0 \\
\hline & Ophiostoma querci & 11 & 3 & 0 \\
\hline & Phomopsis spp. & 0 & 3 & 10 \\
\hline & Umbelopsis isabellina & 9 & 0 & 0 \\
\hline
\end{tabular}

a Green highlighted blocks indicate the most recovered isolate percentage for each inoculation period.

b Pink highlighted blocks indicate the $2^{\text {nd }}$ most recovered isolate percentage for each inoculation period.

c Aqua highlighted blocks indicate the $3^{\text {rd }}$ most recovered isolate percentage for each inoculation period.

\section{SECTION 4: Ouercus coccinea V Inoculation}

Figures 50-52 illustrate the various organisms that were recovered from

inoculated scarlet oak bark during each inoculation period. The V C. parasitica used for the May inoculations was recovered from over $51 \%$ of the bark plugs cultured from the first IP. By the second IP, the recovery of this isolate had decreased to $30 \%$ and by IP-3 the $\mathrm{V}$ isolate was only recovered from $7 \%$ of the samples. As recovery of the $\mathrm{V}$ isolate decreased there was a significant increase in the recovery of other fungi. Most notable was the increase in the recovery of Diplodia corticola which by the second IP was isolated from $45 \%$ of the bark plugs and its recovery decreased to $35 \%$ during the third. As the frequency of $D$. corticola decreased there was a large increase in Trichoderma spp. (47\%) by the third IP. Also, notable is the recovery of the HV C. parasitica isolate from IP's- 1 through 3, ranging from $9 \%, 3 \%$ to $3 \%$ (Table 13 ). 
FIRST INOCULATION PERIOD

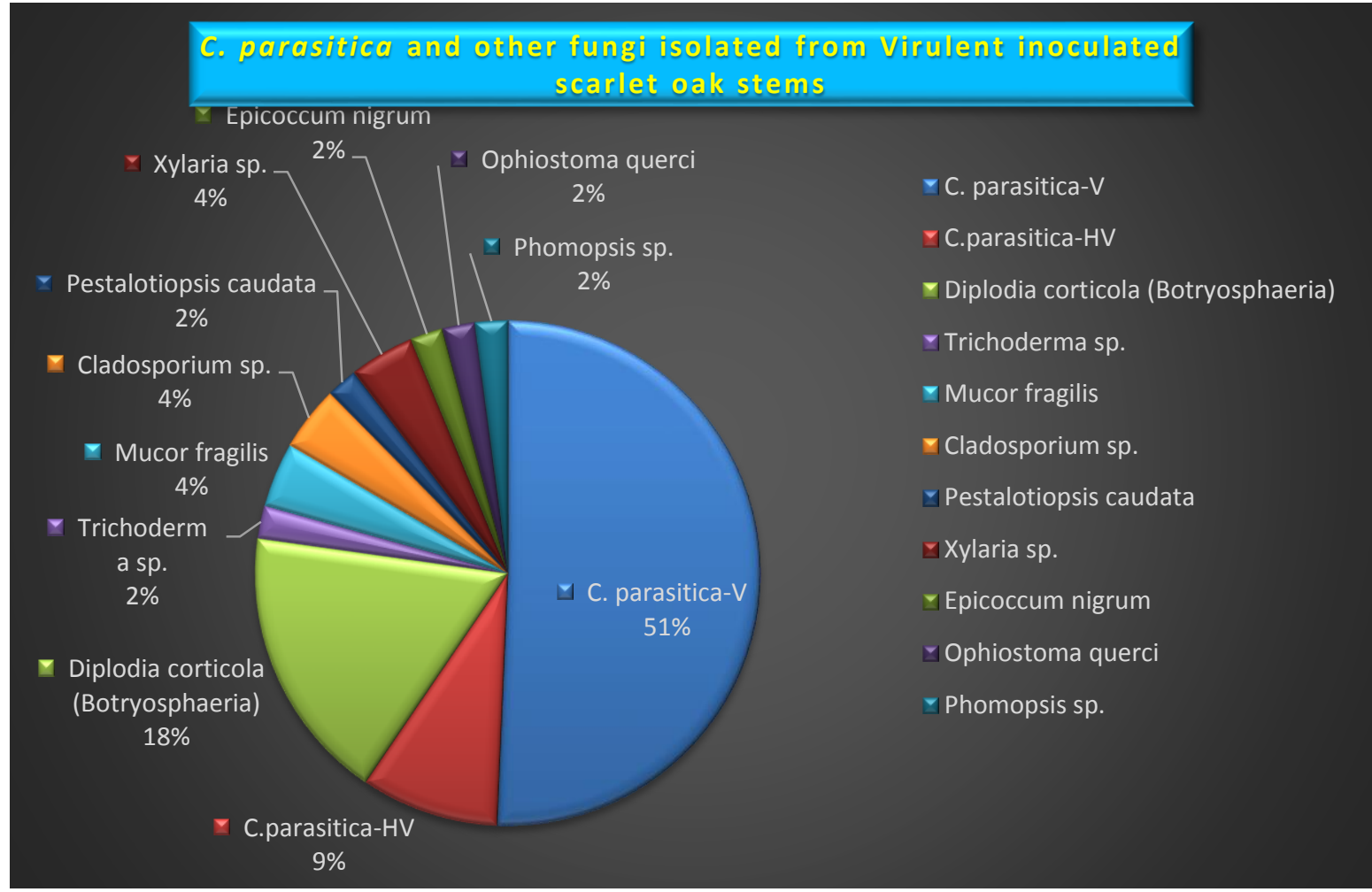

Figure 50: Compilation of fungi recovered from first inoculation period stems and sampled at monthly intervals for the duration of the experiment for the May $20^{\text {th }}$ to December $8^{\text {th }}, 2011$ inoculation period.

\section{SECOND INOCULATION PERIOD}

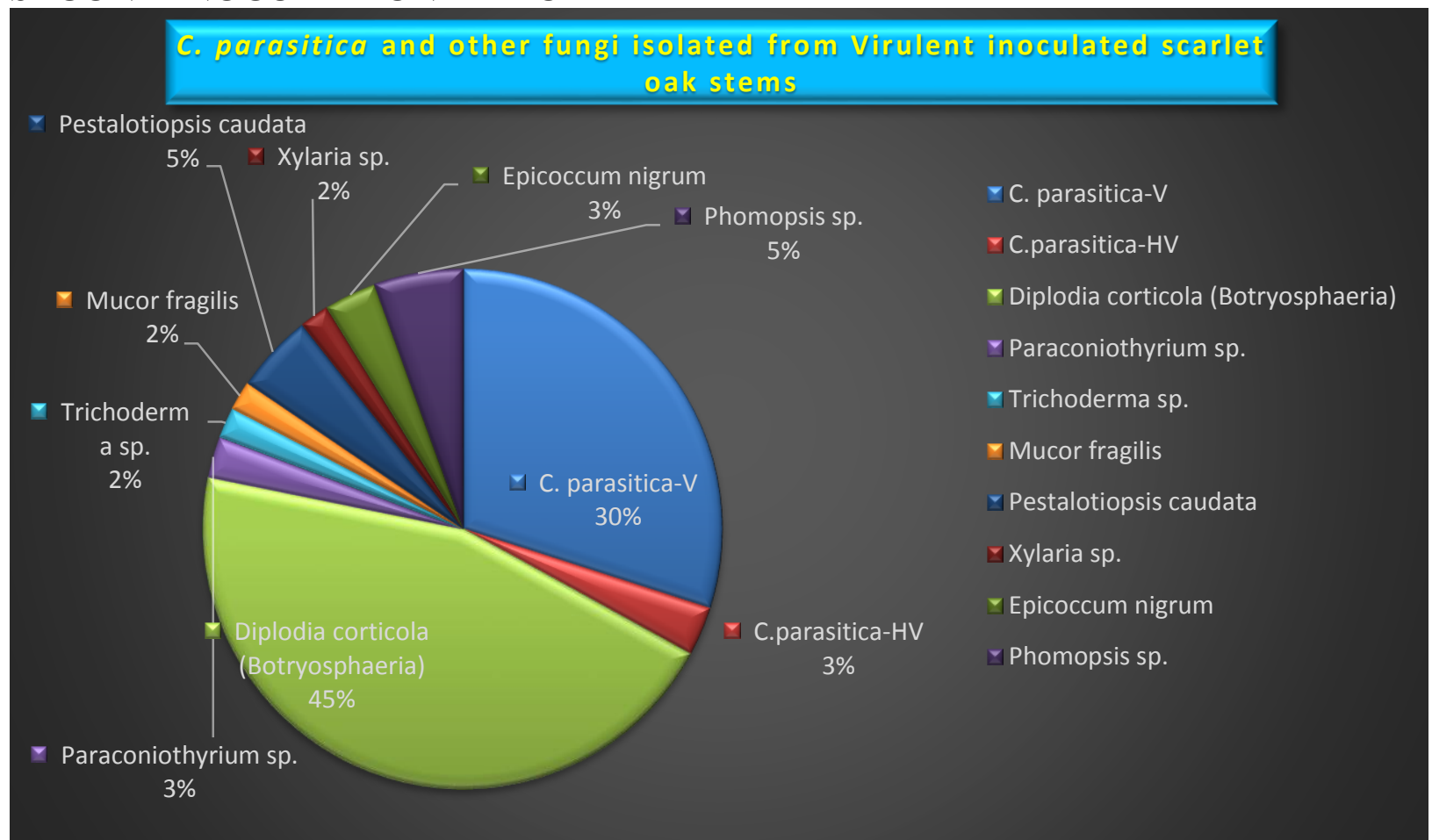

Figure 51: Compilation of fungi recovered from second inoculation period stems and sampled at monthly intervals for the duration of the experiment for the August $4^{\text {th }}$ to December $8^{\text {th }}, 2011$ inoculation period. 
THIRD INOCULATION PERIOD

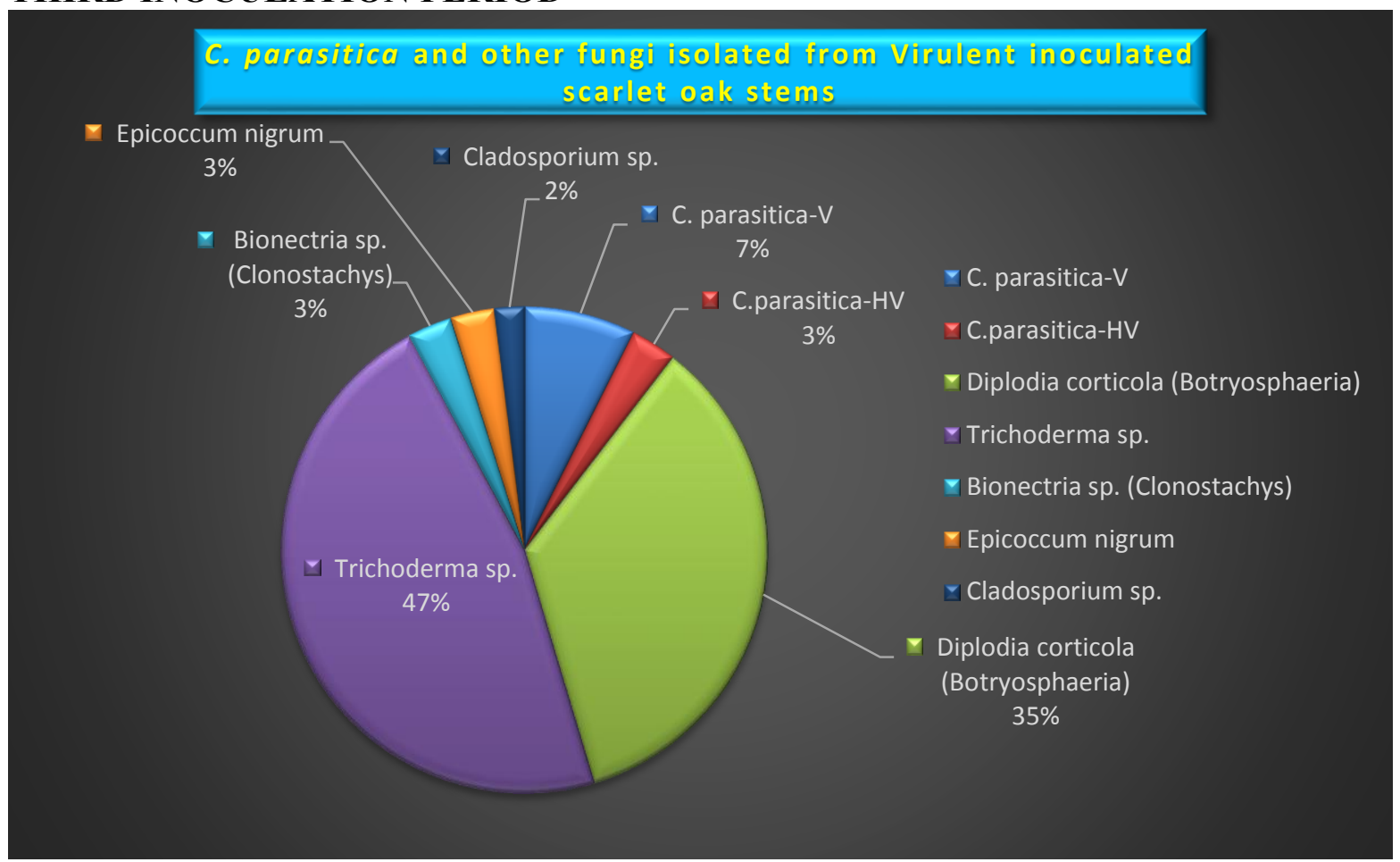

Figure 52: Compilation of fungi recovered from third inoculation period stems and sampled at monthly intervals for the duration of the experiment for the October $4^{\text {th }}$ to December $8^{\text {th }}, 2011$ inoculation period.

TABLE 13: Inoculation period comparison of fungi recovered from stem infections and sampled at monthly intervals for the duration of the experiment for $\mathrm{V}$ inoculated Quercus coccinea.

\begin{tabular}{|l|l|l|l|l|l|}
\hline $\begin{array}{l}\text { Stem/Inoc } \\
\text { Type }\end{array}$ & Fungal Type $^{\text {a }}$ & Inoc 1 & Inoc 2 & Inoc 3 \\
\hline \begin{tabular}{l} 
Q. coccinea-V \\
\hline
\end{tabular} & & $(\%)$ & $(\%)$ & $(\%)$ \\
\hline & C. parasitica-V & $51^{\mathrm{a}}$ & 30 & 7 \\
& C.parasitica-HV & $9^{\mathrm{c}}$ & 3 & 3 \\
\hline & $\begin{array}{l}\text { Diplodia corticola } \\
\text { (Botrysphearia spp. })\end{array}$ & $18^{\mathrm{b}}$ & 45 & 35 \\
\hline & Paraconiothyrium spp. & 0 & 3 & 0 \\
\hline & Trichoderma spp. & 2 & 2 & 47 \\
\hline & $\begin{array}{l}\text { Bionectria spp. } \\
\text { (Clonostachys spp.) }\end{array}$ & 0 & 0 & 3 \\
\hline & Mucor fragilis & 4 & 2 & 0 \\
\hline & Cladosporium spp. & 4 & 0 & 2 \\
\hline & Pestalotiopsis caudata & 2 & 5 & 0 \\
\hline & Xylaria spp. & 4 & 2 & 0 \\
\hline & Epicoccum nigrum & 2 & 3 & 3 \\
\hline & Ophiostoma querci & 2 & 0 & 0 \\
\hline & Phomopsis spp. & 2 & 5 & 0 \\
\hline & Umbelopsis isabellina & 0 & 0 & 0 \\
\hline
\end{tabular}

${ }^{a}$ Green highlighted blocks indicate the most recovered isolate percentage for each inoculation period.

b Pink highlighted blocks indicate the $2^{\text {nd }}$ most recovered isolate percentage for each inoculation period.

c Aqua highlighted blocks indicate the $3^{\text {rd }}$ most recovered isolate percentage for each inoculation period. 
SECTION 5: Quercus coccinea HV inoculation

When the HV C. parasitica isolate was used to inoculate the scarlet oak stems the fungi recovered were similar to those recovered from the V inoculated stems (Figures 53-55). Again, with each sample period fewer samples yielded the HV isolate. For the May inoculation, the HV isolate was recovered from $66 \%$ of the bark plugs cultured from the first IP. By the second IP, the recovery of this isolate had decreased to $19 \%$ and by IP-3 the HV isolate almost remained the same at 20\%. As recovery of the HV isolate decreased there was a significant increase in the recovery of other fungi. Most notable was the increase in the recovery of $D$. corticola which by the second IP was isolated from $33 \%$ of the bark plugs and decreased to $18 \%$ during the third. The reduction in recovery of $D$. corticola was accompanied by a large increase in Trichoderma spp. (54\%) by IP-3. The V C. parasitica isolate also was recovered from the first (13\%) and second (2\%) inoculation periods but was not recovered for the third (Table 14).

\section{FIRST INOCULATION PERIOD}

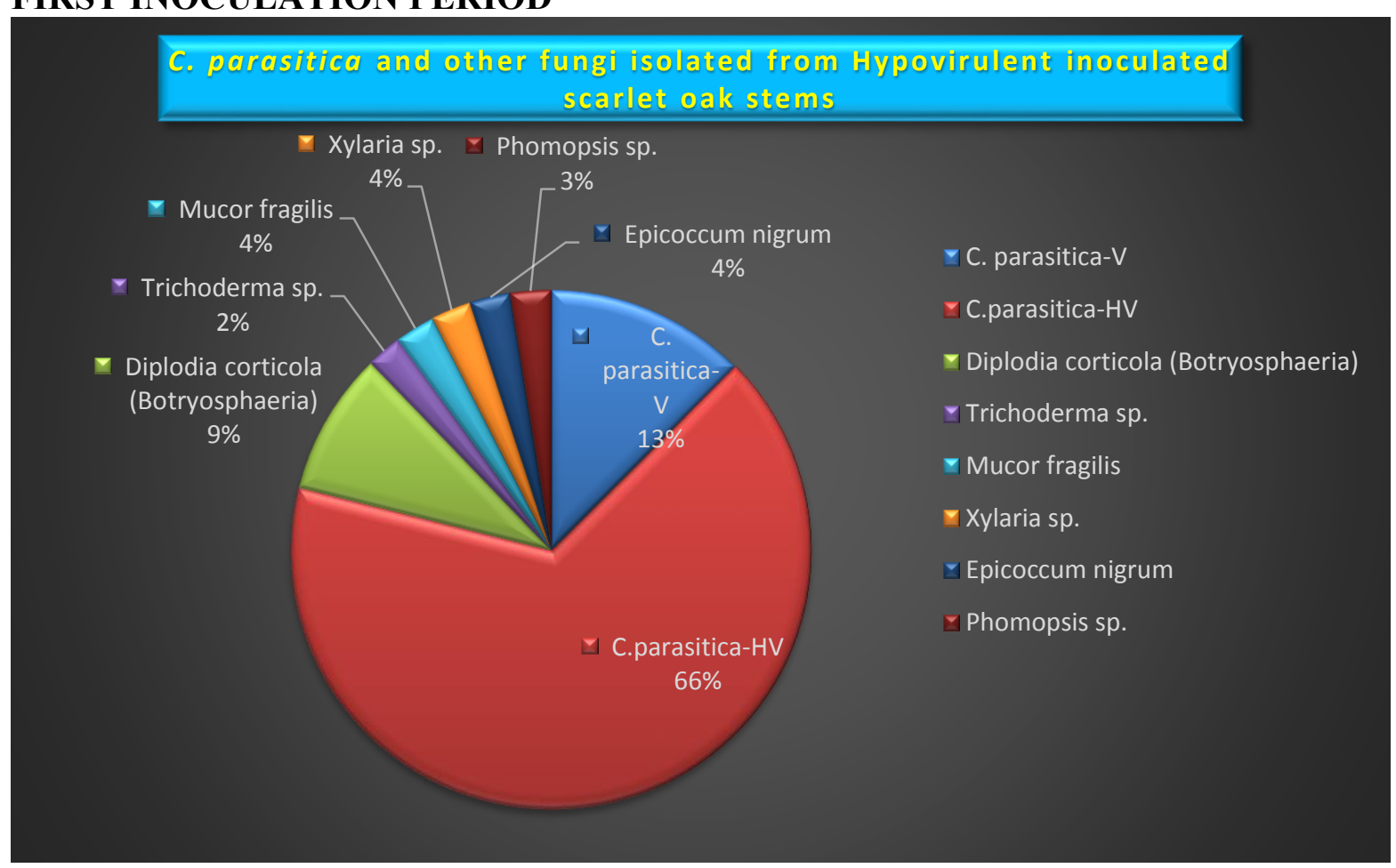

Figure 53: Compilation of fungi recovered from first inoculation period stems and sampled at monthly intervals for the duration of the experiment for the May $20^{\text {th }}$ to December $8^{\text {th }}, 2011$ inoculation period. 
SECOND INOCULATION PERIOD

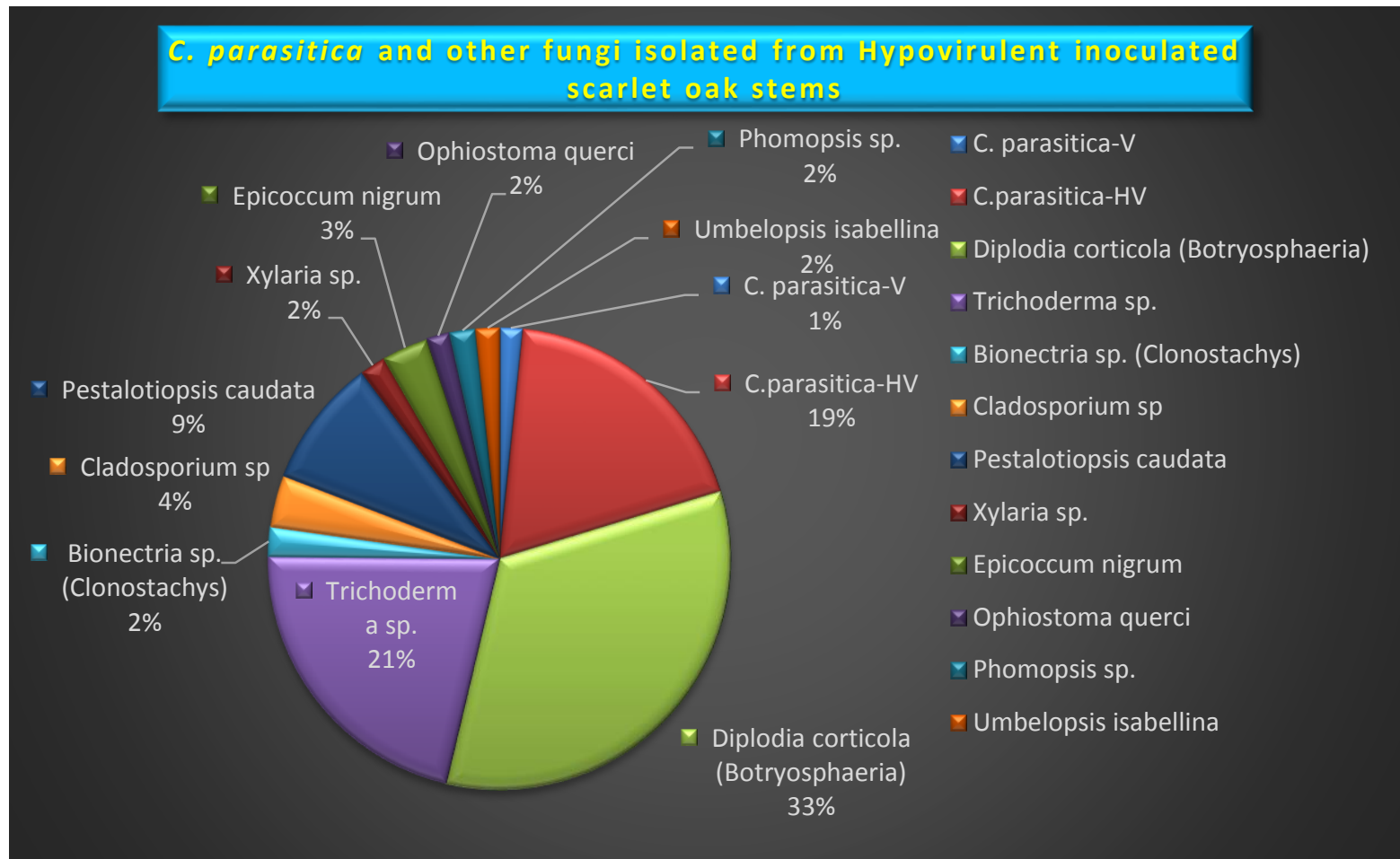

Figure 54: Compilation of fungi recovered from second inoculation period stems and sampled at monthly intervals for the duration of the experiment for the August $4^{\text {th }}$ to December $8^{\text {th }}, 2011$ inoculation period.

\section{THIRD INOCULATION PERIOD}

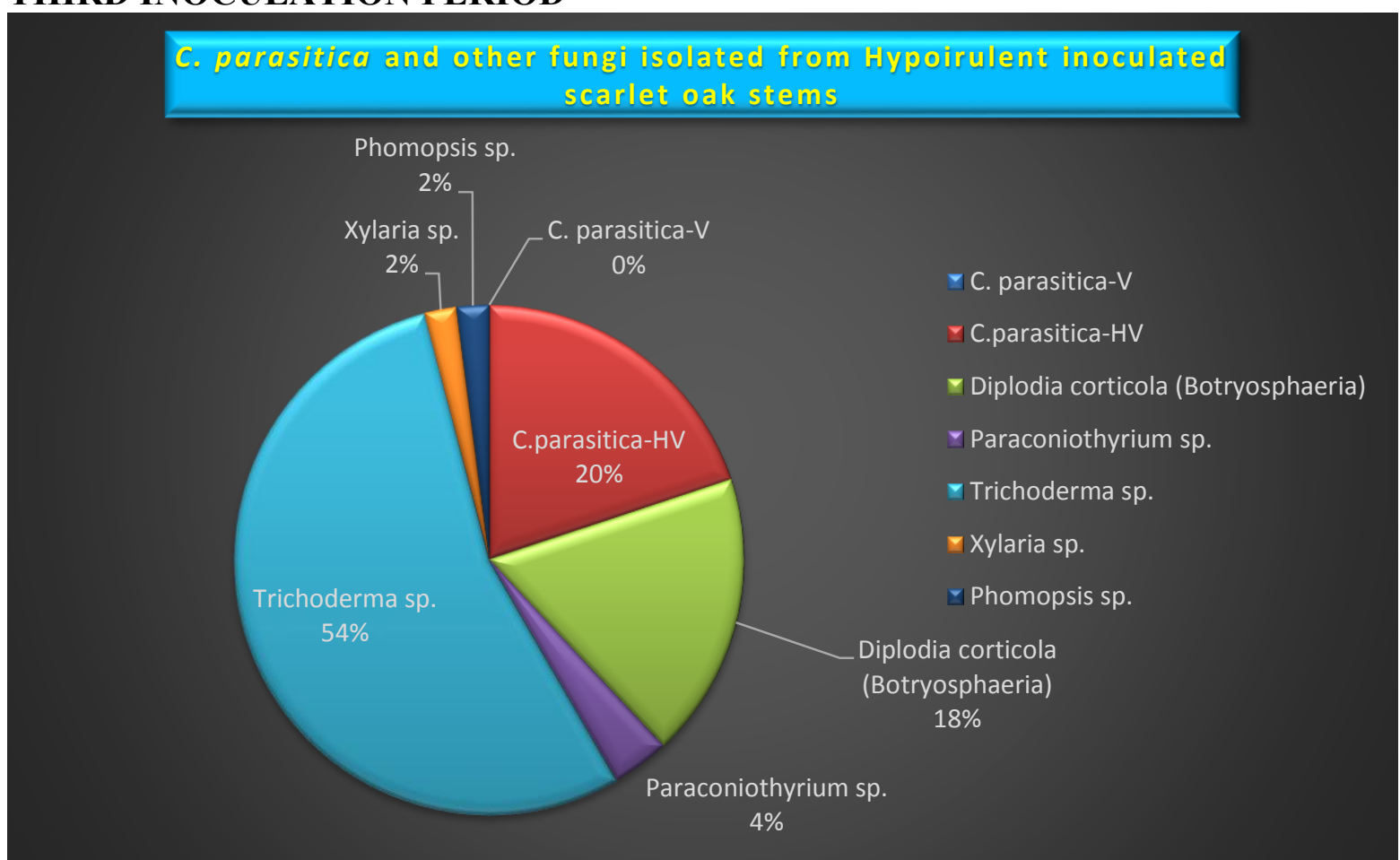

Figure 55: Compilation of fungi recovered from third inoculation period stems and sampled at monthly intervals for the duration of the experiment for the October $4^{\text {th }}$ to December $8^{\text {th }}, 2011$ inoculation period. 
TABLE 14: Inoculation period comparison of fungi recovered from stem infections and sampled at monthly intervals for the duration of the experiment for HV inoculated Quercus coccinea.

\begin{tabular}{|l|l|l|l|l|l|}
\hline Stem/Inoc Type & Fungal Type $^{\text {a }}$ & & Inoc 1 & Inoc 2 & Inoc 3 \\
\hline Q. coccinea-HV & & $(\%)$ & $(\%)$ & $(\%)$ \\
\hline & C. parasitica- $\boldsymbol{V}$ & $13^{\mathrm{b}}$ & 2 & 0 \\
\hline & C. parasitica-HV & $66^{\mathrm{a}}$ & 19 & 20 \\
& $\begin{array}{l}\text { Diplodia corticola } \\
\text { (Botrysphearia spp.) }\end{array}$ & $9^{\mathrm{c}}$ & 33 & 18 \\
\hline & Paraconiothyrium spp. & 0 & 0 & 4 \\
\hline & Trichoderma spp. & 2 & 21 & 54 \\
\hline & $\begin{array}{l}\text { Bionectria spp. } \\
\text { (Clonostachys spp.) }\end{array}$ & 0 & 2 & 0 \\
\hline & Mucor fragilis & 4 & 0 & 0 \\
\hline & Cladosporium spp. & 0 & 4 & 0 \\
\hline & Pestalotiopsis caudata & 0 & 9 & 0 \\
\hline & Xylaria spp. & 4 & 2 & 2 \\
\hline & Epicoccum nigrum & 4 & 3 & 0 \\
\hline & Ophiostoma querci & 0 & 2 & 0 \\
\hline & Phomopsis spp. & 3 & 2 & 2 \\
\hline & Umbelopsis isabellina & 0 & 2 & 0 \\
\hline
\end{tabular}

a Green highlighted blocks indicate the most recovered isolate percentage for each inoculation period. b Pink highlighted blocks indicate the $2^{\text {nd }}$ most recovered isolate percentage for each inoculation period.

c Aqua highlighted blocks indicate the $3^{\text {rd }}$ most recovered isolate percentage for each inoculation period.

\section{SECTION 6: Quercus coccinea (Water Agar) Control Inoculation}

When water agar was used to inoculate the scarlet oak stems, the fungi other than C. parasitica cultured from these isolates were similar to those recovered from the $\mathrm{V}$ and HV inoculated stems (Figures 56-58). Though not inoculated, the $\mathrm{V}$ isolate was recovered from the second (4\%) and third (12\%) inoculation periods. Also, HV isolates were not recovered for the first two IP's, but were recovered at 10\% for IP-3. The most prominent species recovered was Trichoderma spp., increasing from 37\%, 49\% to 53\% from the three IP's. The second most prominent species switched among the inoculation periods between Cladosporium spp. (15\%), O. querci (11\%), and Trichoderma spp. (15\%) throughout all three inoculation periods, respectively (Table 15). 
FIRST INOCULATION PERIOD

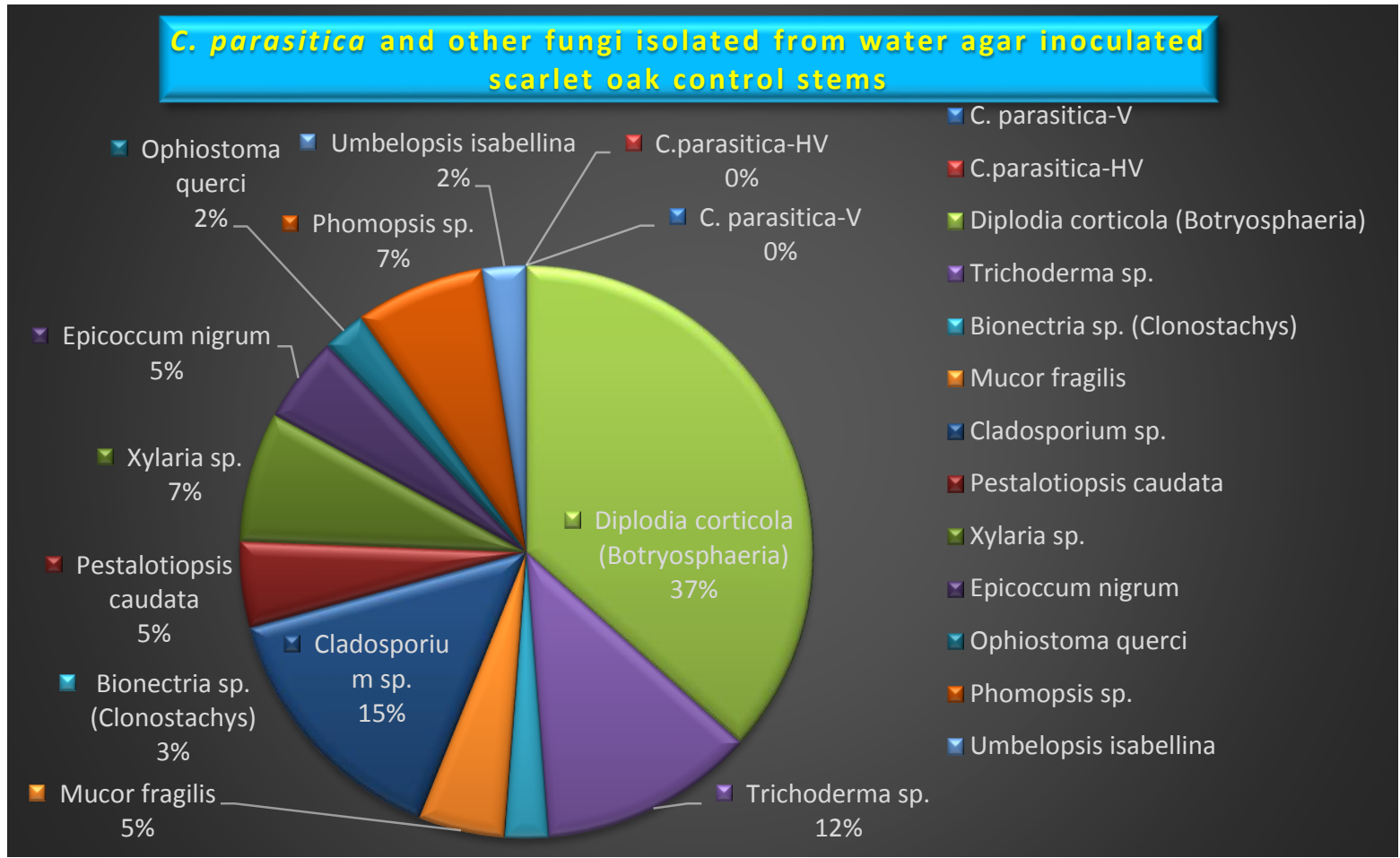

Figure 56: Compilation of fungi recovered from first inoculation period stems and sampled at monthly intervals for the duration of the experiment for the May $20^{\text {th }}$ to December $8^{\text {th }}, 2011$ inoculation period.

\section{SECOND INOCULATION PERIOD}

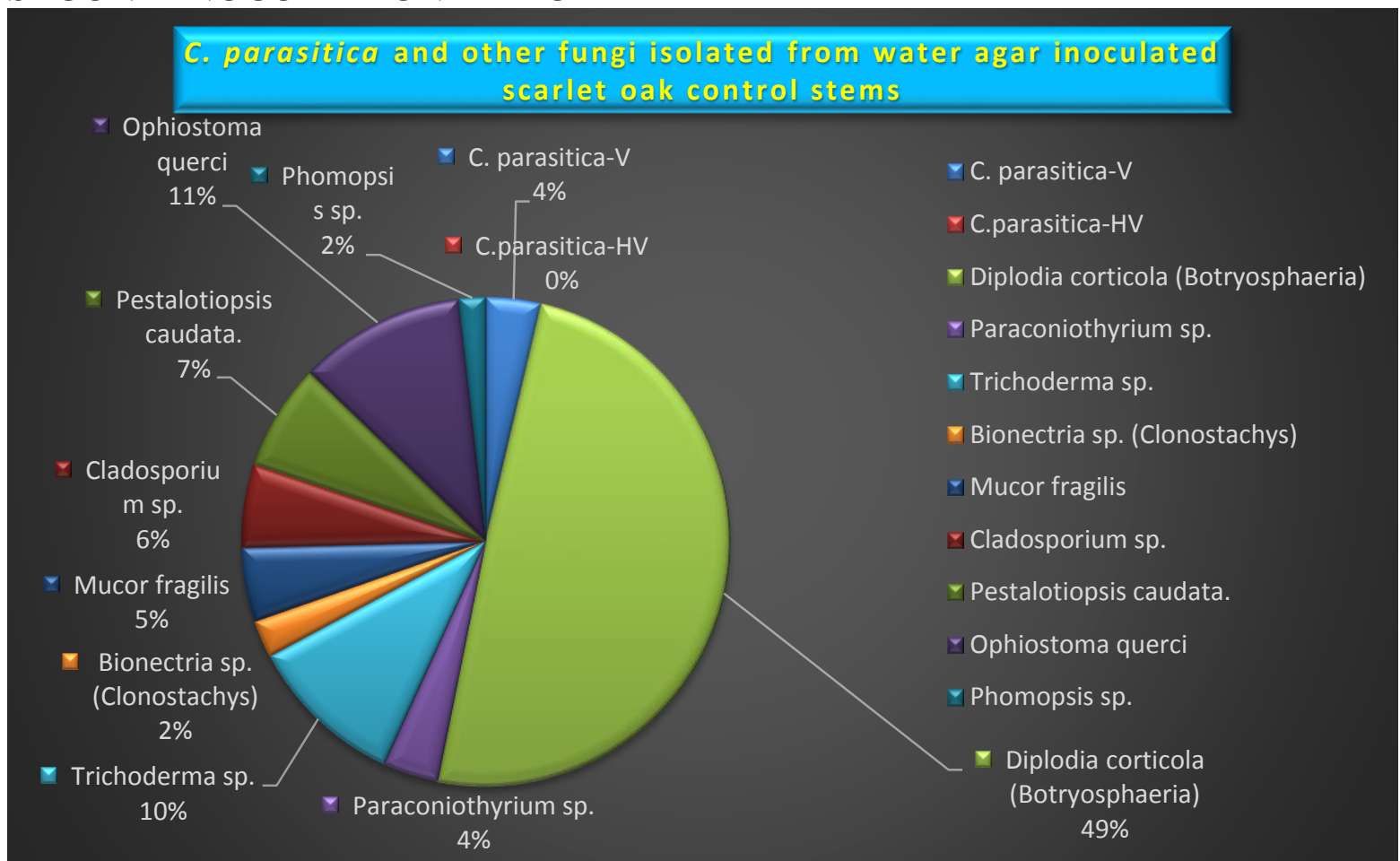

Figure 57: Compilation of fungi recovered from second inoculation period stems and sampled at monthly intervals for the duration of the experiment for the August $4^{\text {th }}$ to December $8^{\text {th }}, 2011$ inoculation period. 
THIRD INOCULATION PERIOD

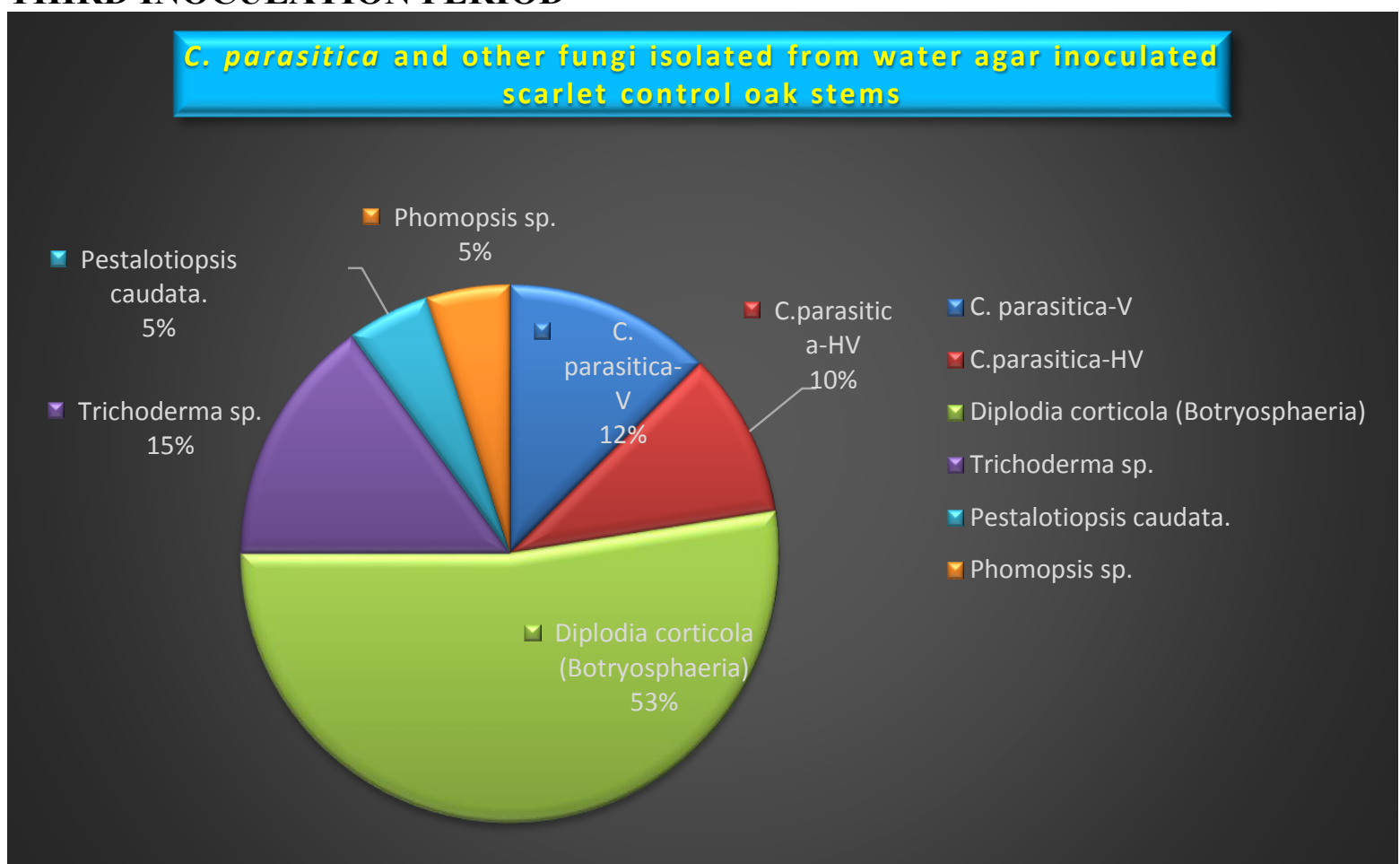

Figure 58: Compilation of fungi recovered from third inoculation period stems and sampled at monthly intervals for the duration of the experiment for the October $4^{\text {th }}$ to December $8^{\text {th }}, 2011$ inoculation period.

TABLE 15: Inoculation period comparison of fungi recovered from stems and sampled at monthly intervals for the duration of the experiment for Water Ager inoculated Quercus coccinea.

\begin{tabular}{|l|l|l|l|l|l|}
\hline $\begin{array}{l}\text { Stem/Inoc } \\
\text { Type }\end{array}$ & Fungal Type & Inoc 1 & Inoc 2 & Inoc 3 \\
\hline $\begin{array}{l}\text { Q. coccinea- } \\
\text { Control }\end{array}$ & & $\mathbf{( \% )}$ & $\mathbf{( \% )}$ & $\mathbf{( \% )}$ \\
\hline & C.parasitica-V & 0 & 4 & 12 \\
& C.parasitica-HV & 0 & 0 & 10 \\
\hline & $\begin{array}{l}\text { Diplodia corticola } \\
\text { (Botrysphearia spp.) }\end{array}$ & 37 & 49 & 53 \\
& Paraconiothyrium spp. & 0 & 4 & 5 \\
\hline & Trichoderma spp. & 12 & 10 & 15 \\
\hline & $\begin{array}{l}\text { Bionectria spp. } \\
\text { (Clonostachys spp.) }\end{array}$ & 3 & 2 & 0 \\
\hline & Mucor fragilis & 5 & 5 & 0 \\
\hline & Cladosporium spp. & 15 & 6 & 0 \\
\hline & Pestalotiopsis caudata & 5 & 7 & 5 \\
\hline & Xylaria spp. & 7 & 0 & 0 \\
\hline & Epicoccum nigrum & 5 & 0 & 0 \\
\hline & Ophiostoma querci & 2 & 11 & 0 \\
\hline & Phomopsis spp. & 7 & 2 & 5 \\
\hline & Umbelopsis isabellina & 2 & 0 & 0 \\
\hline
\end{tabular}

${ }^{a}$ Green highlighted blocks indicate the most recovered isolate percentage for each inoculation period.

b Pink highlighted blocks indicate the $2^{\text {nd }}$ most recovered isolate percentage for each inoculation period.

c Aqua highlighted blocks indicate the $3^{\text {rd }}$ most recovered isolate percentage for each inoculation period. 


\section{SECTION 7: $C$. parasitica Isolated External to Observable Infection}

When isolates were cultured from beyond the perceived visual boundaries of $C$. parasitica colonized bark tissue, results indicated that the observations of the area were accurate. The first inoculation period was the only period that external recovery of $\mathrm{V}$ or HV isolates occured. Virulent $C$. parasitica was recovered at $3 \%$ on chestnut but not from $\mathrm{V}$ inoculated oak stems. However, the $\mathrm{V}$ isolate was recovered at $5 \%$ from $\mathrm{HV}$ inoculated oak. The HV isolate also was recovered at a 5\% rate from chestnut but not from oak HV inoculated stems for the first inoculation period. The second and third inoculation periods had no $\mathrm{V}$ or $\mathrm{HV}$ isolates recovered within 5-cm external to inoculation sites. Individual species of fungi other than $C$. parasitica were tallied but not identified. Cultured isolate results for this evaluation were recorded only as positive $(+)$ or negative (-) for C. parasitica.

\section{DISCUSSION}

This experiment was designed to determine if the saprophytic phase of $C$. parasitica could contribute significantly to the production of inoculum. Of particular interest is whether hypovirulent strains might colonize and sporulate as well or better on a dead host. If dead stems could contribute significantly to the pool of HV inoculum they might enhance the potential for biological control.

The fungal strains BRV-1 and BRHV-1 used were collected from the research site. The American chestnut stems also were cut at the site from a previously established plantation. A hypovirulent isolate (Euro-7) had been used previously at this plantation. The BRHV-1 isolate that was recovered from the site expressed similar dsRNA banding patterns upon extraction (Figure C). Some scarlet oak stems were cut from the test site and others from the WVU forest. These two tree species occur in similar Appalachian 
Mountain habitats. Scarlet oak becomes infected but tolerates natural C. parasitica infections and therefore was selected as a second host. Torsello (1994) showed that heavier sporulation may occur on scarlet oak after an infected host tree dies. Therefore, scarlet oak was included to evaluate its potential to increase HV inoculum production.

The experimental design measured colonization and sporulation differences among $\mathrm{V}$ and $\mathrm{HV}$ on the two hosts. Total colonization was not measured as the area of an elipse due to disproportional colonization patterns and variable shapes of the advancing colonies. Therefore colonization was measured in $\mathrm{cm}^{2}$ based on half of a rectangle to account for the variable mycelial growth patterns. This variance of colony advancement was not typical of the elliptical, diffuse canker formed on live trees. The reasons for this are likely associated with the loss of active resistance and callus formation in dead stems not suppressing mycelial advancement at the leading edge. Other organisms also may have played a role in guiding the $C$. parasitica colonization do to their presence within the bark. Cryphonectria parasitica colonies advanced around the other fungi when possible. Layer effect within stacks and location effect among spatially separated groups also were measured. The design assessed mycelial colonization and stroma formation for three different inoculation periods (IP's). Another factor evaluated was other fungi, which were or became resident occupants, of the infection site throughout the experiment.

The hypothesis of this experiment was that $C$. parasitica will colonize and sporulate as well on dead scarlet oak as American chestnut when artificially inoculated. It also was hypothesized that the HV fungus can grow and sporulate successfully on both hosts as a saprophyte. Further evaluated, as an ancillary part of the experiment, was if HV strain would disseminate to other non-HV inoculated stems. 
While much is known about $C$. parasitica as a pathogen, very little is known about its saprophytic capabilities. In an important precurser to this study, Prospero et al., (2006) evaluated the saprophytic stage of V and HV C. parasitica from naturally obtained infections in pre-existing firewood and experimental stacks of European chestnut (C. sativa). Prospero's study measured hypovirus infected conidia rates and showed that stacks of non-inoculated HV infected stems can effectively produce HV inoculum. The methods for the current experiment took snapshots over time of inoculated V and HV strains as they colonized artificial wounds initiated at bi-monthly intervals following the death of the stem pieces on two hosts.

The saprophytic colonization and sporulation on scarlet oak was considered important because the natural active resistance within living scarlet oak to $C$. parasitica may diminish when it dies (Torsello, 1994). When scarlet oak is healthy it appears to have the ability to obtain and support colonization of $C$. parasitica without being killed unless additional stress factors are added to its environment such as drought, insect attack or other pathogens. This can be seen in living, infected, mature scarlet oak in nature that has swollen butts and bole cankers but are otherwise healthy (Appendix Figure 62). This experiment examined the ability of $C$. parasitica to colonize this host once active resistance was eliminated. Whether scarlet oak could bolster C. parasitica's HV spore production also was an important consideration of the experiment.

This leads to another important aspect of the research. On living American chestnut trees, V C. parasitica colonizes and sporulates more than HV. Active resistance is not readily evident in $\mathrm{V}$-infected American chestnut and the tree is girdled and dies as a result of infection. The HV fungus generally allows the tree to respond by producing callus tissue and prevents death from girdling. As a result, sporulation is generally reduced and less HV spores are produced on living infected trees. This experiment 
examined the ability of dead oak and chestnut stems to produce and serve as an HV inoculum source. The fungus grows readily on living or dead chestnut but scarlet oak also could be an important source of HV inoculum if HV inoculum is produced in abundance once scarlet oak dies. Since American chestnut is now less common in our forests, scarlet oak may be an important contributor to HV inoculum production.

\section{COLONIZATION and SPORULATION}

Even though colonization and sporulation analysis were presented separately they will be discussed together. The results indicate the two factors are related. Though no formal statistical test was performed for correlations, some comparisons were made. Also, no analysis was made among the inoculation periods. This was primarily due to the amount of time that each IP was able to be measured. Measurement periods varied from six measurements for IP-1, to four measurements for IP-2 and two measurements for IP3. Measurements ceased due to the onset of winter and the natural deterioration of the bark. No comparisons were made during this experiment between live and dead trees of either host. All inoculations were made on stems that were cut from living trees a week prior to the experiment and the first inoculation period (IP).

In general, the increase in fungal colonization coincided with increased sporulation. The ability of $C$. parasitica to colonize bark and sporulate diminished with each successive inoculation period. The findings further support the observation that scarlet oak provided a better substrate for colonization and sporulation during inoculation periods 2 and 3 than did American chestnut. Stems with larger areas of infected tissue also tended to produce more stroma. Though, there were several incidences when HV isolates sporulated more than V, especially in the second and third IP's, typically V isolates colonized more area and sporulated more than HV isolates. When V was 
compared to $\mathrm{HV}$ sporulation, a colonized region of equal size did not always reflect equal stroma production. HV generally sporulated less, but this was not always the case. When $\mathrm{V}$ to $\mathrm{HV}$ colonization were evaluated $\mathrm{V}$ grew better than $\mathrm{HV}$ in all instances other than in the third IP when HV inoculated chestnut stems grew better than V chestnut. Significantly greater fungal colonization for the V fungus occurred only in the first IP for oak and chestnut. Thereafter, colonization of $\mathrm{V}$ was higher than HV but not significantly so for the second and third IP's. Evidence, from IP's 2 and 3, supports less differences in HV colonization when compared to V. Sporulation coincided with these findings except when there was a significant difference in $\mathrm{V}$ and $\mathrm{HV}$ sporulation on oak when it exceeded that on chestnut for IP-2. Sporulation was relatively equal for all treatments during IP-3. Inoculation periods 2 and 3 also revealed more colonization and sporulation on oak for $\mathrm{V}$ and HV. Although V and HV sporulated more during IP-1 on chestnut than oak, both isolates colonized more total area on oak during all IP's.

Layer effect tested the position of inoculated stems relative to their distance from the ground in a stack. In a few instances, differences existed that could suggest a trend for some inoculation periods but there was no overall consistency. No layer indicated better colonization or sporulation even though an effect was expected due to higher moisture levels near the ground. Results for layers mirrored those of total colonization. Scarlet oak grew and sporulated more than chestnut, independent of layers, during IP's 2 and 3. Layers with the highest and lowest colonization and sporulation reversed with regular occurrence for all treatments. The only phenomena that stood out was the unexpected colonization differences between inoculation periods 2 and 3 . In a few instances, HV grew and sporulated better than $\mathrm{V}$ within the same layer and inoculation period. Also, the same layer at times exhibited more colonization or sporulation during IP-3 than IP-2. These circumstances occurred for both V and HV inoculated stems. A 
number of factors may have accounted for the layer effect to lack consistency ranging from; a limited distance from the ground, bark thickness, other saprophytes and moisture content. However, the most likely candidate is simply distance from the ground. That is, the top layer of the stack from the ground was not much more than $50-60 \mathrm{~cm}$ above the lowest layer. Even though L-1 (closest to the ground) was separated from direct ground contact by sourwood stems, environmental conditions within the stack did not vary greatly enough to have accounted for a more distinct effect.

The location effect of the stacks showed no consistent trends throughout the inoculation periods other than that G-1 grew more for all treatments except $\mathrm{HV}$ during IP-3. Like total colonization and sporulation, colonization and stroma production lessened from IP-1 through IP-3 for both the V and HV isolates. As with the effect of layers, in a few instances, IP-3 expressed greater colonization and sporulation than IP-2. Statistically different comparisons indicated that V and HV stems in G-1 tended to grow the most other than for one case in G-2 HV for IP-3. Variation fluctuated among the groups when evaluating sporulation and no discernable trend was evident. Another notable occurrence was that in a couple of instances within IP-3, HV had greater colonization and sporulation than the $\mathrm{V}$ isolate. Location did not play a big role in this study for several reasons. The stacks at their greatest distance were only 33 meters apart, under the same overstory so that shading, humidity, elevation and microclimate were similar. Any slight site differences were not enough to create the conditions necessary to significantly affect colonization and sporulation.

\section{OTHER ORGANISMS}

Saprophytic organisms other than $C$. parasitica played a significant role in the experiment. Prior colonization within the bark tissue, antagonism and myco-parasitism 
all may have contributed to the other organisms' ability to influence the colonization and sporulation of $C$. parasitica. This portion of the study identified the most commonly isolated fungi from within the region of infected bark tissue.

No samples were taken prior to stem cutting. The first bark samples were collected one month after inoculation and then each month throughout the duration of the experiment for all IP's. The percentages of sampled fungi that are presented, represent cumulative numbers for each IP (Figures 41-58). In general, other fungal saprophytes increased and $C$. parasitica (V and HV) decreased with each successive IP. An important observation, was that $\mathrm{V}$ and HV were recovered at nearly $80 \%$ during IP-1. The recovery rates of V and HV were approximately half of that and then half again for IP's-2 and 3, respectively. Therefore, even as other fungi colonized the stem, the bark already colonized by $C$. parasitica during IP-1 consistently yielded high recovery rates of $C$. parasitica throughout the experiment.

The fungal species that commonly occurred on both American chestnut and scarlet oak were identified (Tables 8-15). The community of organisms was similar for both hosts. Most were members of the Ascomycota except Mucor fragilis and Umbelopsis isabellina which are in the phylum Zygomycota and order Mucorales. These two species are common saprophytes and were found in low percentages in IP's - 1 and 2 but infrequently in IP-3. The other ascomycete fungi also were found mostly in the first and second IP's with a decline in diversity toward the third IP. The exceptions were Trichoderma spp. (Hypocreales) and Diplodia corticola (Botrysphaeriales). These two species, even though recovered in low percentages during IP-1, became the most prominent species by IP-2. In the case of the water agar inoculated control stems, the other fungal species that were recovered was consistent with the V and HV inoculated stems. However, in contrast to the C. parasitica inoculated stems, Trichoderma spp. on 
chestnut and D. corticola on oak, were the most prominent isolates recovered from the control stems during IP-1. Both of these species would increase in subsequent inoculation periods. Every treatment type on chestnut showed Trichoderma spp. as the most recovered species by the end of IP-3. This also was the case for scarlet oak, with the exception of the control stems, where $D$. corticola was the most prominent species recovered by the final inoculation period. When $\mathrm{V}$ and $\mathrm{HV}$ were inoculated, $C$.

parasitica was the most prominent species only for IP-1. Thereafter, C. parasitica was recovered less frequently during the remaining inoculation periods than the other fungi.

Various factors may be responsible for the dominance of Trichoderma and $D$. corticola by the second and third IP's. As with all the fungi commonly associated with these stems, they may have been present in some form before the experiment began. Diplodia corticola is a pathogen of oak causing cankers, dieback, stem girdling and sudden death. This fungus also is a facultative necrotroph, the same as C. parasitica, and is commonly found as a successful saprophyte in nature. Trichoderma $s p$. is a known antagonist and produces secondary metabolites responsible for inhibiting the colonization of other microorganisms. Also, Trichoderma spp. are known for their ability to be mycoparasites and successful saprophytes (Campanile, et al., 2007). Therefore, multiple functions exist that support the reduced recovery of $C$. parasitica when these two species also were present. Both $C$. dentata and $Q$. coccinea are members of the Fagaceae and were infected by many of the same organisms. As a pathogen and facultative necrotroph, D. corticola also may have already been present in both hosts. Prior colonization by $D$. corticola could lend an advantage over C. parasitica saprophytically. Campanile, et al., (2007) showed that Trichoderma viride is moderately successful as a biocontrol agent against D. corticola on oak. These factors combined for Trichoderma spp. and D. 
corticola, as well as their strong saprophytic capabilities, lend support to them being the most commonly recovered fungi during the second and third inoculation periods.

Other fungi that were commonly isolated also may grow better than C. parasitica as saprophytes. Epicoccum nigrum (Capnodiales), has the ability to act as a biocontrol agent against brown rot of peaches. The fungus has been shown to be antagonistic and prevent colonization of Monilinia spp. when fruit were pretreated with E. nigrum (Larena et al., 2004). Therefore, it also could have an antagonistic effect against $C$. parasitica. The other remaining resident fungi are classically regarded as general saprophytes. Therefore, once other saprophytes were established in the bark tissue they may have prevented the colonization and sporulation by $\mathrm{V}$ and $\mathrm{HV}$ when $C$. parasitica was introduced during the second and third IP's. The results indicate that C. parasitica is a poor competitor when not introduced shortly after the host's death, as the fungus shows limited ability to grow and sporulate on previously colonized, dead host tissue.

\section{TREE HOSTS}

When comparisons were made between $C$. dentata and $Q$. coccinea, scarlet oak proved to be a better substrate for saprophytic colonization and sporulation of $\mathrm{V}$ and $\mathrm{HV}$ over time. Though chestnut had better V and HV colonization and sporulation initially during IP-1, oak supported better colonization and stroma production for IP's -2 and 3 . A few factors may have contributed to this situation. There was a limited quantity of non-infected $60 \mathrm{~cm}$ lengths of American chestnut which limited the number of suitable stems for this experiment. Therefore, the diameter and age of the stems between chestnut and oak were inconsistent. All chestnut stems were cut from young saplings between approximately five-to-ten centimeters in diameter that were grown in an open light regime. The scarlet oak stems were from a shaded understory. Therefore, the oak 
growth was more suppressed and generally the stems were much older than the chestnut stems. As a result, oak had thicker, older and more durable bark than the chestnut. Sporulation may have been better on chestnut during the first two months for IP-1 because $C$. parasitica is a pathogen of chestnut, but the bark condition also may have influenced stroma production. The infections were much more evident on the chestnut initially and the thinner bark allowed for stroma to erupt though the epidermal layer more rapidly. Although the infected oak bark would discolor orange, fewer stroma would initially erupt from the bark (Figure 4). When this occurred the stroma would appear through thin cracks in the bark until colonization was better established (Appendix Figure 61). This thicker oak bark may have afforded $C$. parasitica more available substrate in the presence of other competing organisms and better nutritional content allowing for more colonization and sporulation for the second and third IP's. The thinner bark of chestnut also deteriorated and lost moisture more rapidly. By the end of the experiment, the oak bark was still relatively intact while the chestnut bark was beginning to flake and peel off like paper. Future saprophytic experimentation between these two tree species would be well served by using stems of the same age, bark thickness and grown under similar conditions.

Though infections occur naturally, specific conditions may be required for optimal colonization and sporulation to take place. The time of year death and subsequent infection occur are likely critical colonization factors. Spring infection, when the host is beginning active growth, may be important as indicated by the IP-1 results. Hosts that are infected in late summer or early fall may not allow C. parasitica to grow and sporulate as well. Infection prior to death also could result in more inoculum production. This could be an especially important factor for scarlet oak that can seemingly tolerate and survive $C$. parasitica infections. Naturally occurring infections 
may not be as common on scarlet oak as chestnut. However, if scarlet oak is already infected and then dies, it could be a good contributor to natural inoculum production. Therefore, wounded or recently dead scarlet oak stems within proximity to infected chestnut could better obtain and contribute to inoculum production.

\section{VERSES HV}

Generally, V grew and sporulated more than HV on both hosts. However, some anomalies occurred that may have been due in part to other competitive organisms and colonization as a saprophyte rather than a parasite. In the first IP, V grew and sporulated significantly better for all treatments. This was not the case for IP's - 2 and 3 . Though trends were better for $\mathrm{V}$, colonization and sporulation were no longer significantly greater in all cases for IP-2 and were never significantly different in IP-3. In fact, V and HV were nearly equal in all treatments during IP-3 and HV colonized more area than V on chestnut during IP-3. There also were several incidences that where the analysis of layer and location effect showed that HV grew and sporulated on the same host better than V during the same IP (Tables 2-7).

\section{DISSEMINATION}

Dissemination of the HV strain also was an interesting observation made during the experimentation. Even though care was taken not to cross-contaminate stacks during sampling, the $\mathrm{HV}$ isolate showed up in the $\mathrm{V}$ and Control stacks. Isolates made from four out of five groups had incidences when HV was isolated from a V inoculated stack. Also, when isolations were made from Control stacks, HV was recovered from two of the five stacks. HV infections took place on both oak and chestnut in the V stacks and on oak in the Control stacks. This cross-contamination was especially evident during IP-3 
on oak when $12 \%$ of the $\mathrm{V}$ and $10 \%$ of the $\mathrm{HV}$ samples were isolated in a stack to which they were not introduced. The non-inoculated stems that were placed vertically in the middle of the HV also occasionally became HV infected. HV isolates were recovered from two oak and three chestnut stems. This occurred for at least one positive sample in all HV stacks on oak and chestnut. The V stacks of these vertical stems obtained an HV infection in one isolation from G-5. These findings indicate that HV can disseminate from a saprophytic source and colonize both hosts. It is not known if these HV infections were new or the result of converted V colonies. BRHV-1 was shown to tranmit the hypovirus to over $95 \%$ of all BRV-1 pairings during preliminary tests. Another important observation is that $\mathrm{V}$ was routinely recovered from $\mathrm{HV}$ colonies. This result is typical of the preliminary tests for BRHV-1, where the single spore procedure yielded approximately $20 \%$ to $25 \% \mathrm{~V}$ from an HV colony.

\section{CONCLUSIONS}

The results of this experiment indicate that V and HV C. parasitica can saprophytically grow and sporulate when inoculated into American chestnut and scarlet oak stems. This ability diminishes dramatically over time as the bark tissue is colonized by other fungi. The longer the period between host mortality and inoculation, the less likely it will be for either host to become colonized. This latter result is likely due to other saprophytes already occupying the bark if the host is not infected by $C$. parasitica before the other fungi become well established. Though $C$. parasitica may not be a strong saprophyte, evidence indicates it can grow and sporulate well when inoculated immediately before or after the death of its host. This may simply be due to colonization by other saprophytes that may be competitors. Inoculated $C$. parasitica also is capable of limited colonization and inoculum production on the stems of both hosts up to four 
months after the host stem dies. However, neither V nor HV are competitive with other saprophytes several months after stem death. Scarlet oak was a slightly better host than chestnut when inoculated in this experiment. Thicker bark may have been a large contributor to better colonization and sporulation on the oak host. When inoculated after their death, both hosts are capable of producing HV inoculum. Results from this experiment indicate the potential to use recently cut and inoculated stems of American chestnut and scarlet oak as an HV inoculum source.

\section{FUTURE DIRECTION for EXPERIMENTATION}

Many factors became evident during this research that could be modified to help understand $C$. parasitica as a saprophyte. Future experiments could assess freshly cut stems inoculated during different seasons. The stems for this analysis were not inoculated until late May. Stems that are cut and inoculated at different seasons likely will produce different amounts of inoculum and colonized area. A future experiment could use a monthly stem cutting method to help evaluate the optimal time to produce the most HV inoculum. Dissemination is another potential area of investigation for future research. The HV infections that were observed among the non-HV inoculated stacks could be used in experimentation. HV inoculated stems could be stacked and placed in rings around live hosts to assess how effective $\mathrm{HV}$ inoculum could be when disseminated from this saprophytic source. Live hosts could be wounded by various methods and also left unwounded to act as trees to trap inoculum. V also could then be inoculated at various heights, to test the effectiveness of $\mathrm{HV}$ transmission. The incorporation of live stems into future experiments also could help contrast colonization and sporulation trends as a saprophyte while pointing out the host's influence on C. parasitica. The latter comparison would be especially important for $\mathrm{HV}$ where American chestnut is able to 
respond by callus formation. Live stems also could be inoculated and then girdled at various time intervals and compared with inoculated cut stems to observe differences. The effect of layers within a stack was negligible in this experiment. However, the stacks could be made larger to better observe if the distance from the ground has any influence on inoculum production. There also are other hosts that may serve as effective substrates. All of the variables described above could be influenced by different topography, microclimates and regions that were not included in this experiment.

The saprophytic phase of V and HV C. parasitica remains complex. The results of this research show the potential for $C$. parasitica to produce $\mathrm{HV}$ inoculum saprophytically. I would encourage future studies that measure the potential use of $\mathrm{HV}$ C. parasitica's saprophytic phase in biological control experiments. 


\section{APPENDIX A}

\section{MEDIA INGREDIENTS}

\section{Table 16: Ingredients of Media Used}

\section{1.) Potato Dextrose Agar}

- $39 \mathrm{~g}$ potato dextrose agar

- $100 \mathrm{mg}$ methionine

- $1 \mathrm{~mL}$ stock solution biotin

- $1000 \mathrm{~mL}$ distilled water

\section{2.) Glucose Yeast Extract with antibiotics}

- 10 g glucose

- 2 g yeast extract

- $1 \mathrm{~g}$ potassium phosphate

- 0.5 g magnesium sulfate

- $1 \mathrm{~mL}$ stock solution thiamine

- $1 \mathrm{~mL}$ stock solution biotin

- $2 \mathrm{~mL}$ stock solution microelements

- 20 g agar

- $1000 \mathrm{~mL}$ distilled water

- $50 \mathrm{mg}$ chlorotetracycline (post autoclave)

- $10 \mathrm{mg}$ streptomycine sulfate (post autoclave)

\section{3.) Modified Brome-Cresol Green Medium (Powell, 1995)}

- 24 g PDA

- 3 g Yeast extract

- 4 g Malt extract

- $600 \mathrm{mg}$ Tannic acid

- $50 \mathrm{mg}$ Brome-cresol green

- $12 \mathrm{~g}$ Difco agar

- $0.5 \mathrm{~mL}$ Tween

- $1000 \mathrm{~mL}$ Distilled water

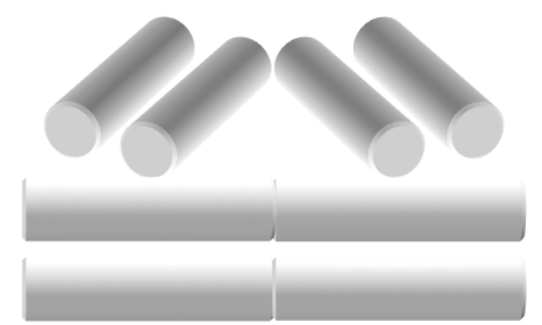

Figure 59: Representing the position and arrangement in one layer of a stacked pile 


\section{$\underline{\text { Additional Photos }}$}

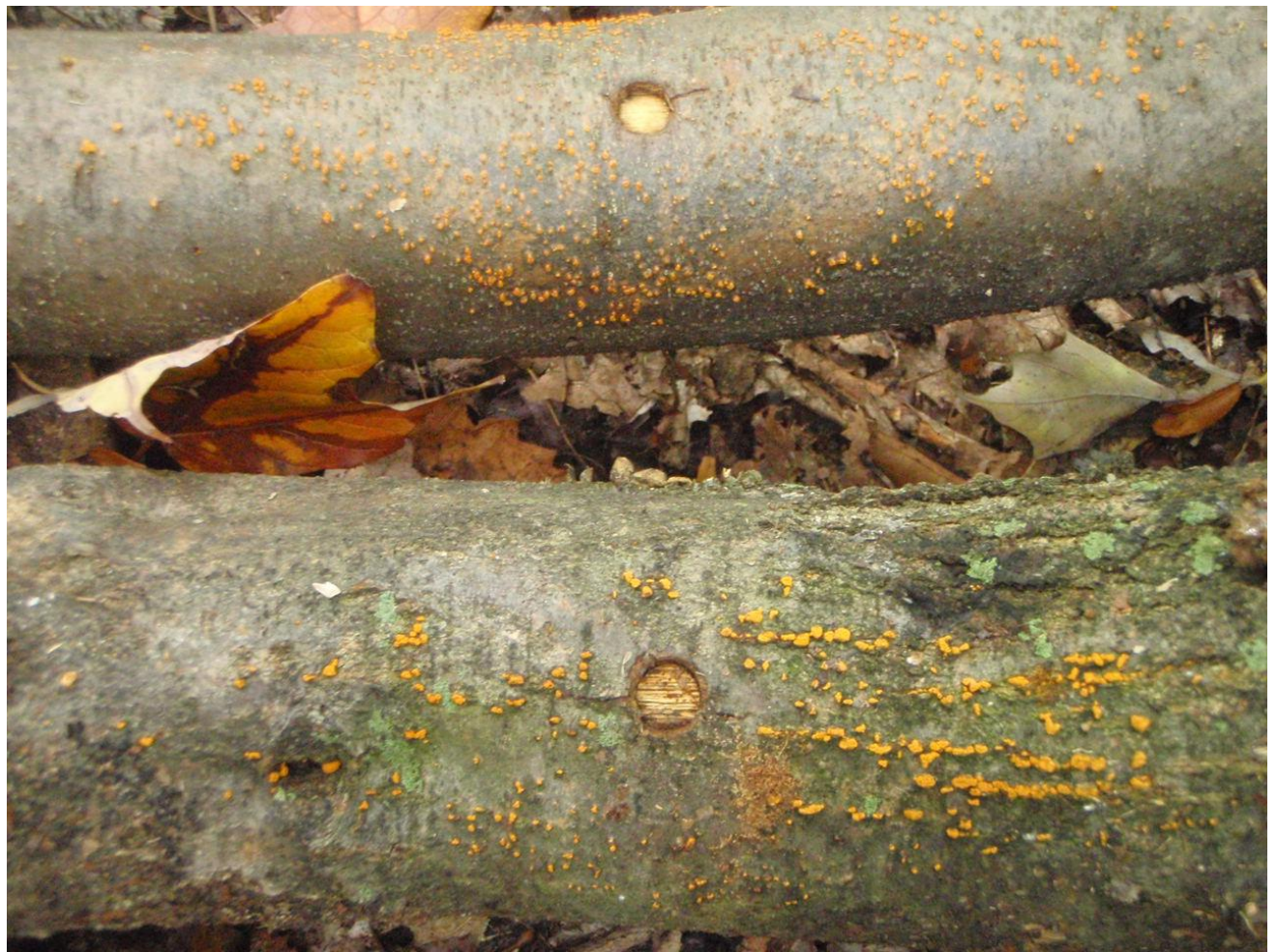

Figure 60: Inoculation site showing colonization and sporulation on American chestnut (top) and scarlet oak

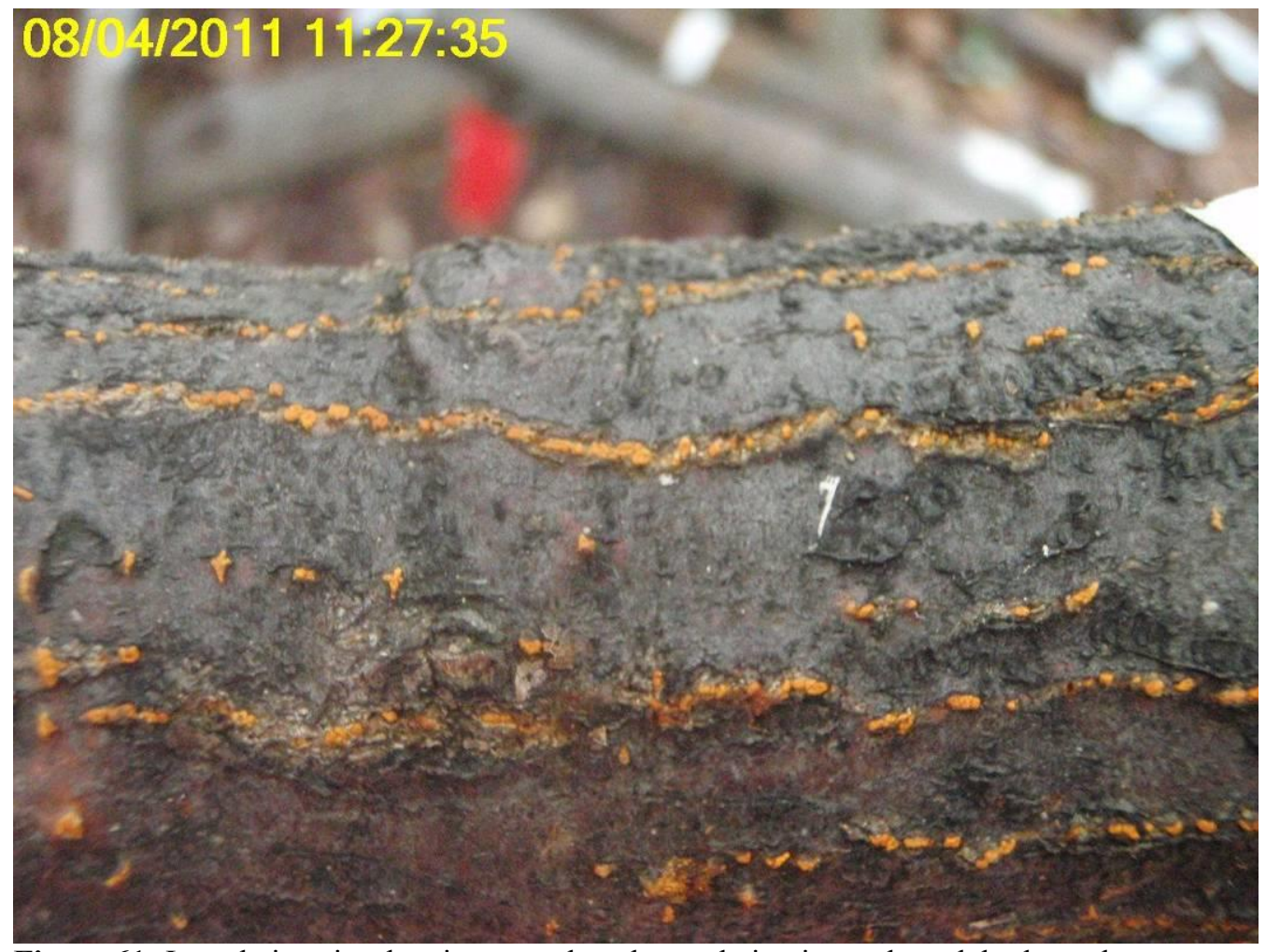

Figure 61: Inoculation site showing growth and sporulation in scarlet oak bark cracks 


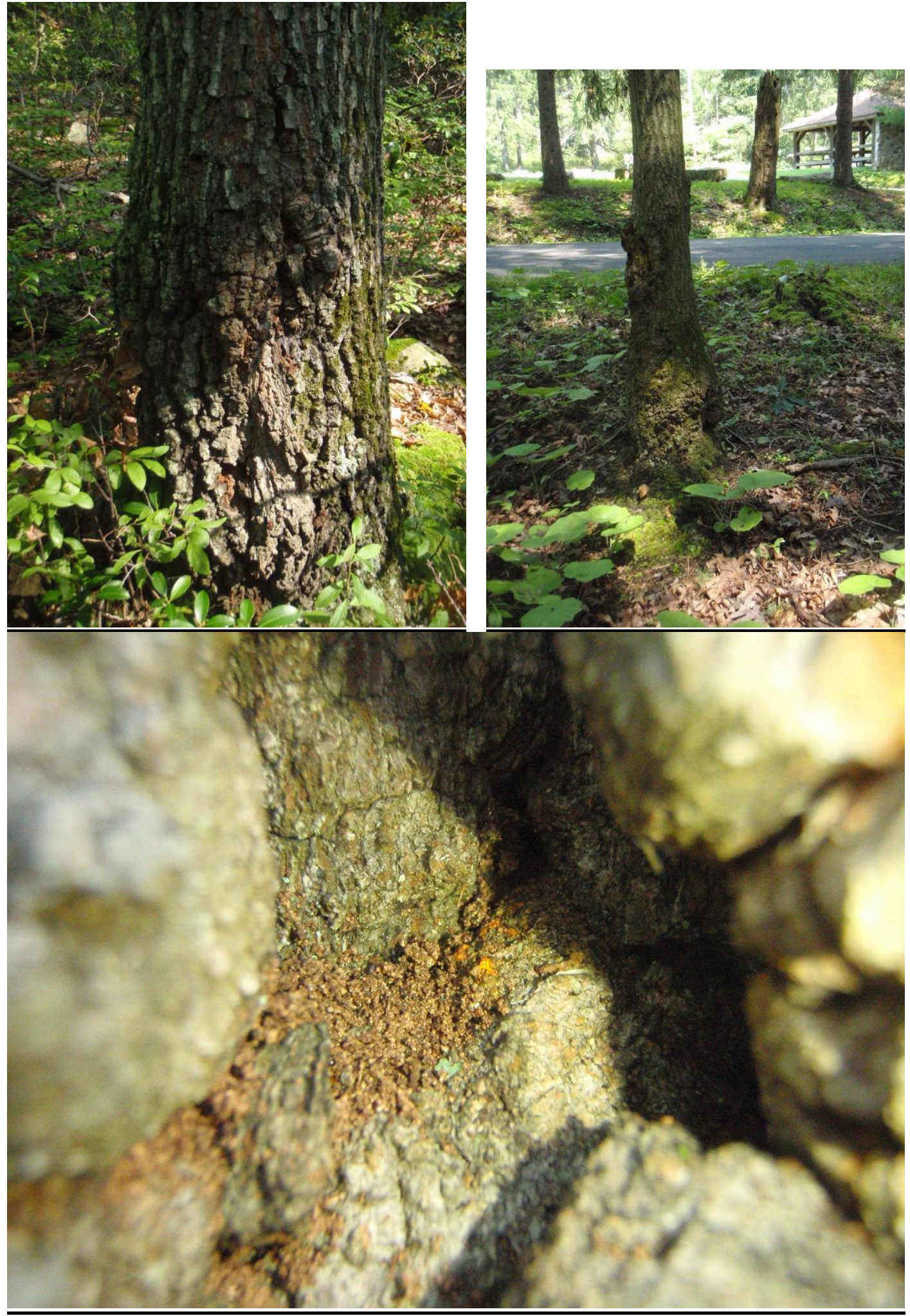

Figure 62: Showing naturally infected scarlet oak with swollen butt, bole infections and stroma 


\section{Other Fungi Isolated}

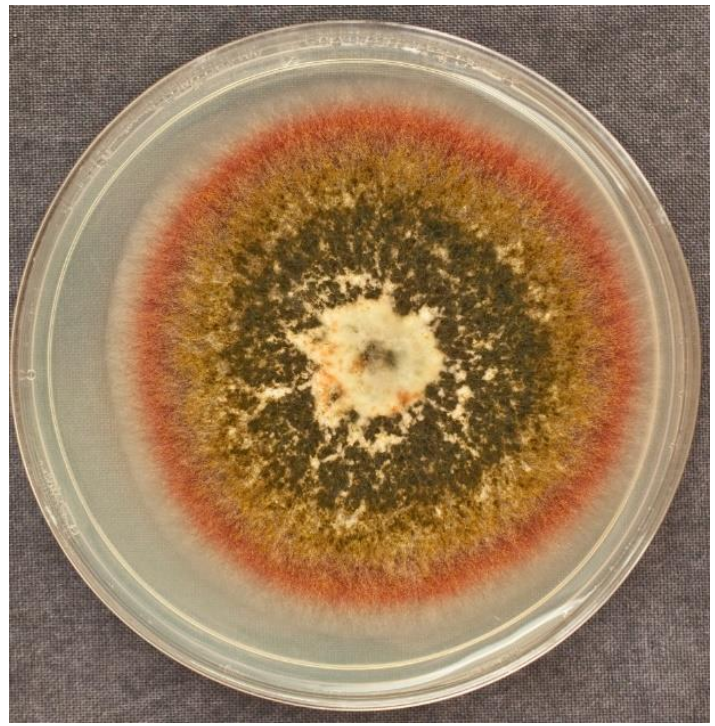

Figure 63: Epicoccum nigrum isolate isolate

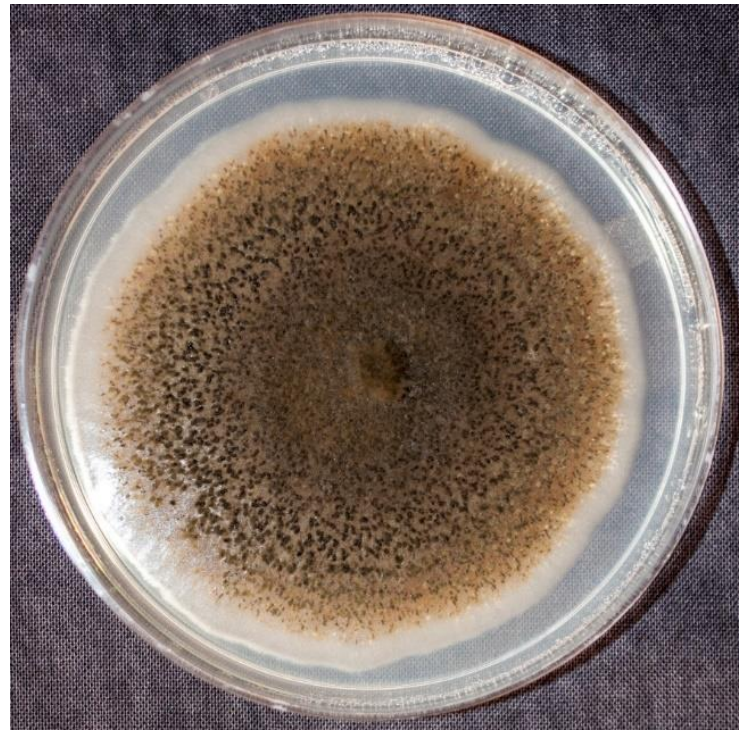

Figure 65: Paraconiothyrium spp. isolate

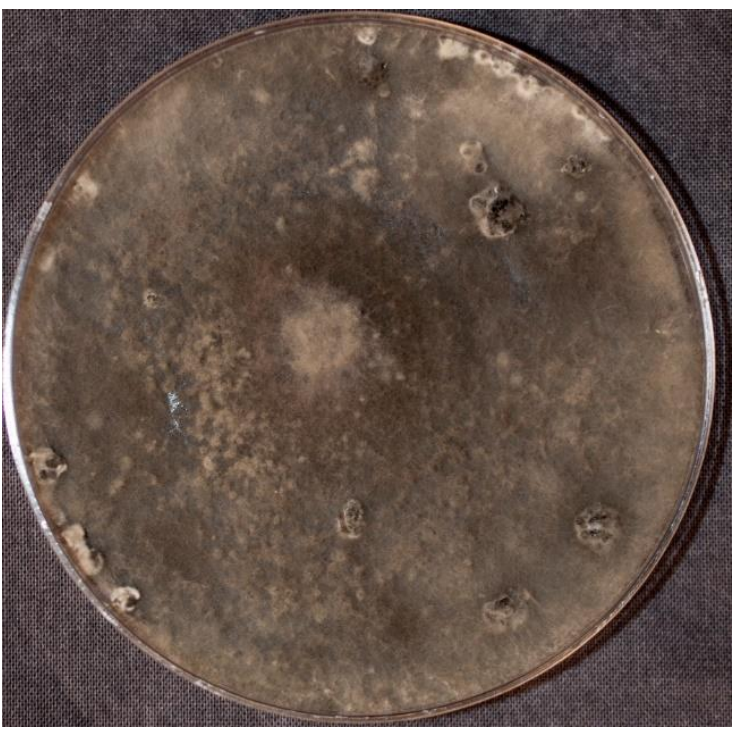

Figure 64: Diplodia corticola (Botryosphaeria spp) isolate

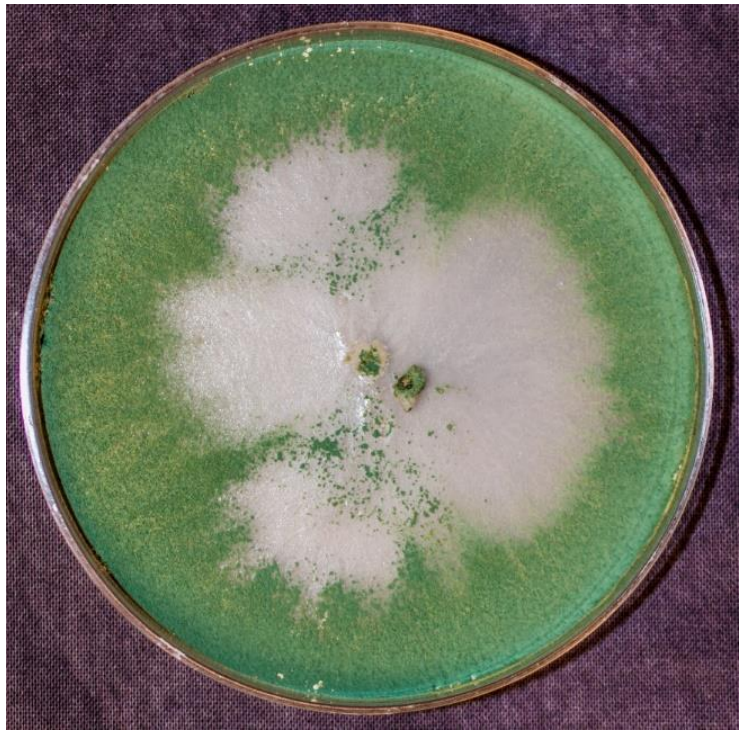

Figure 66: Trichoderma spp. isolate

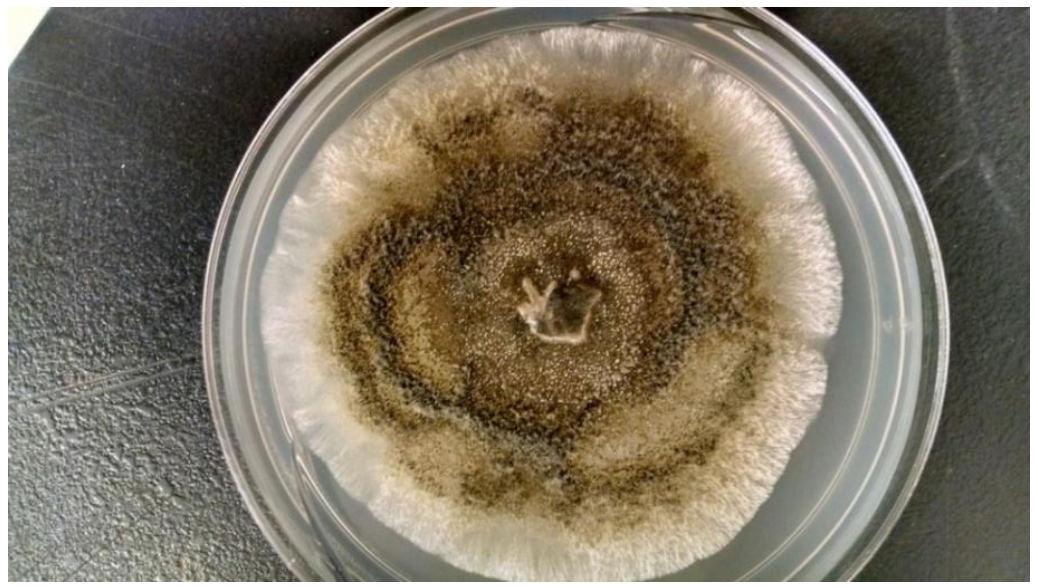

Figure 67: Ophiostoma querci isolate 


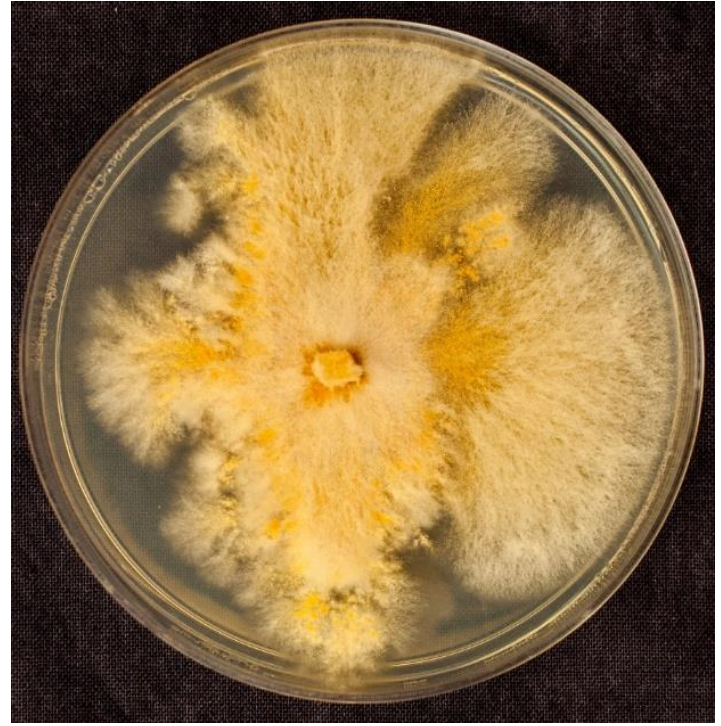

Figure 68: Phomopsis spp. isolate isolate

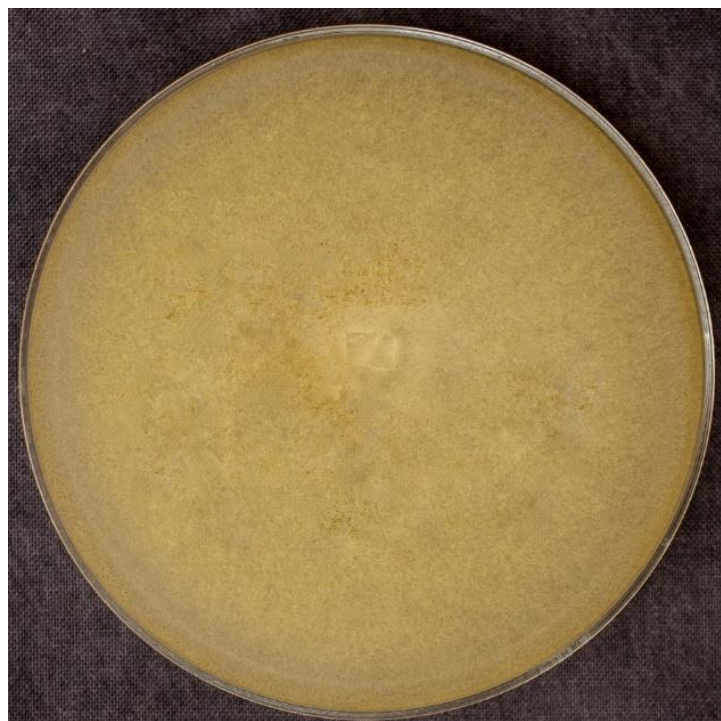

Figure 70: Mucor Fragilis isolate

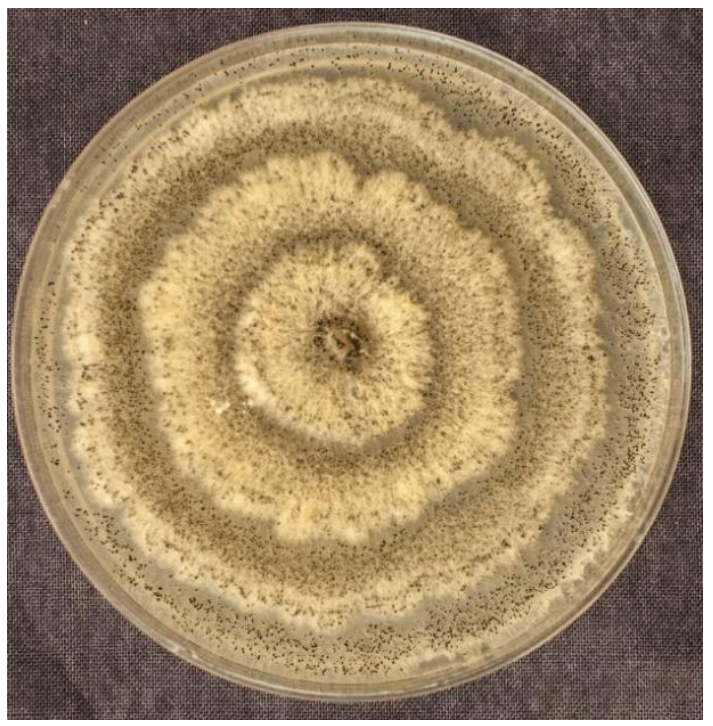

Figure 72: Pestalotiopsis caudata isolate

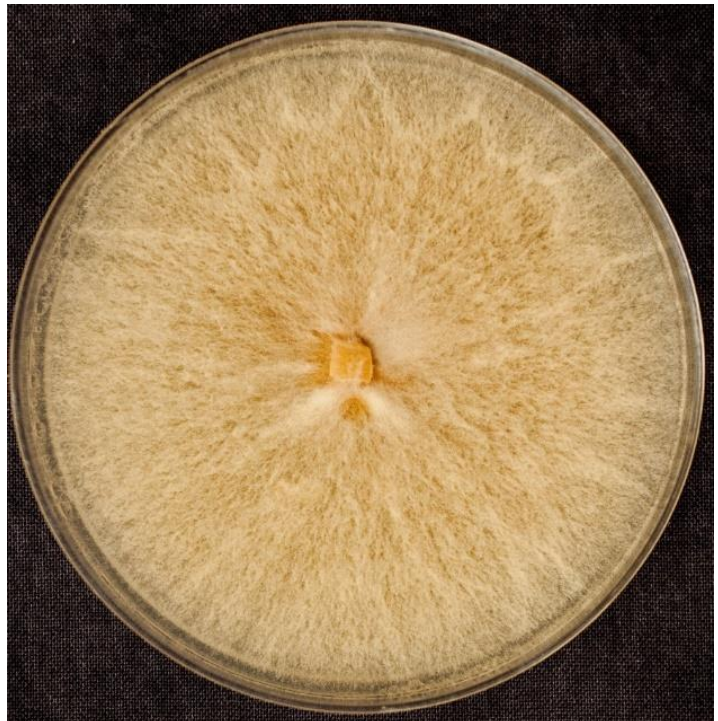

Figure 69: Bionectria spp. (Clonostachys spp.)

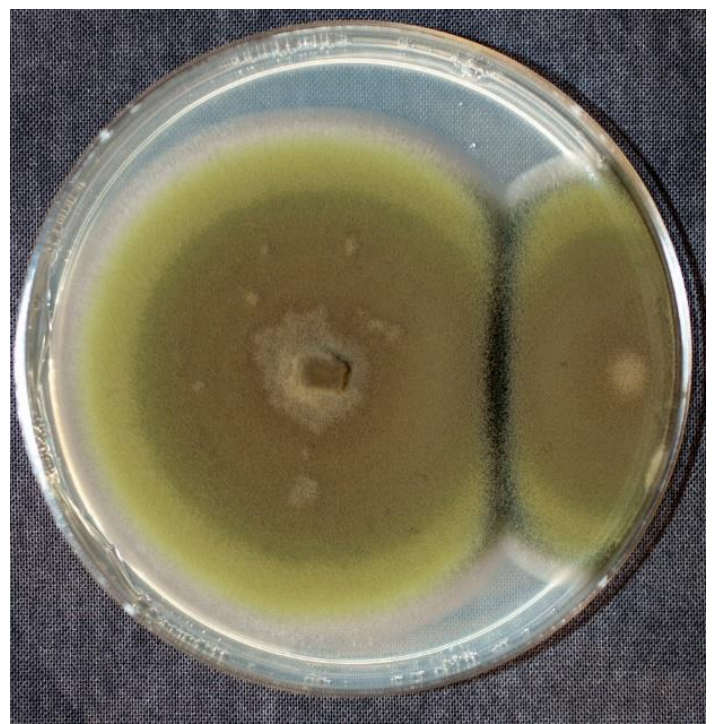

Figure 71: Cladosporium spp. isolate

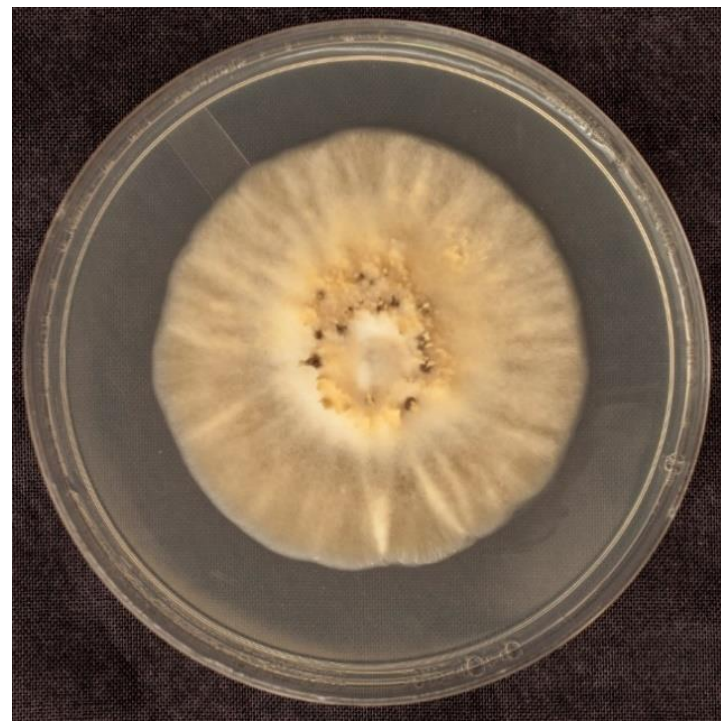

Figure 73: Umbelopsis isabellina isolate 


\section{APPENDIX B}

\section{PCR Products and DNA Sequence Results of Other Isolated Fungi}

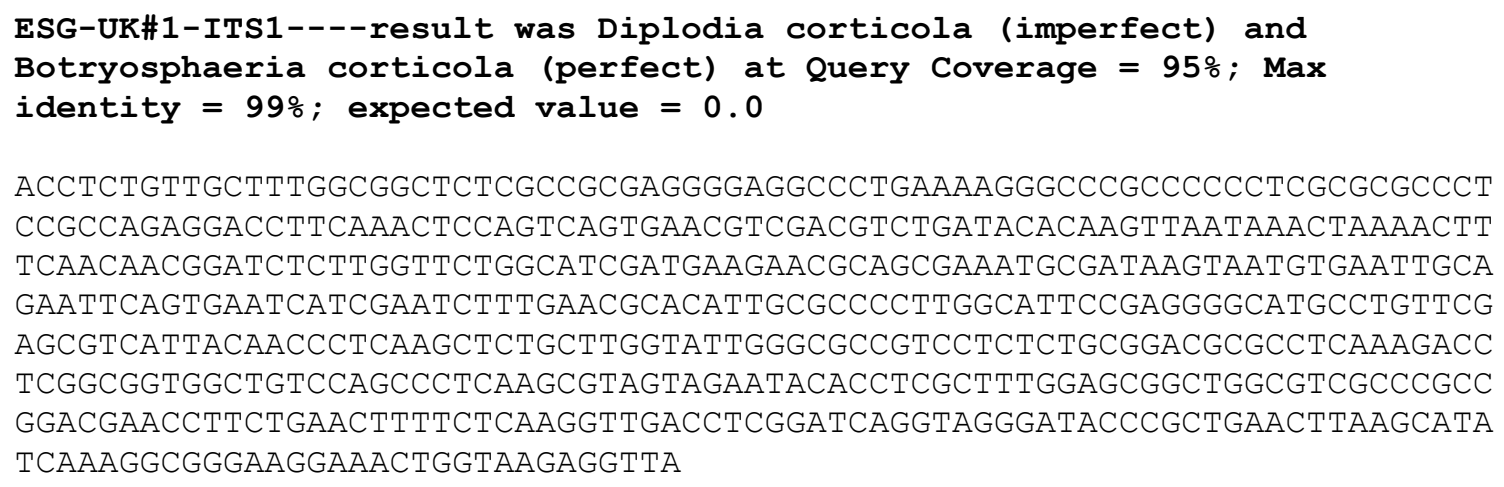

ACCTCTGTTGCTTTGGCGGCTCTCGCCGCGAGGGGAGGCCCTGAAAAGGGCCCGCCCCCCTCGCGCGCCCT CCGCCAGAGGACCTTCAAACTCCAGTCAGTGAACGTCGACGTCTGATACACAAGTTAATAAACTAAAACTT TCAACAACGGATCTCTTGGTTCTGGCATCGATGAAGAACGCAGCGAAATGCGATAAGTAATGTGAATTGCA GAATTCAGTGAATCATCGAATCTTTGAACGCACATTGCGCCCCTTGGCATTCCGAGGGGCATGCCTGTTCG AGCGTCATTACAACCCTCAAGCTCTGCTTGGTATTGGGCGCCGTCCTCTCTGCGGACGCGCCTCAAAGACC TCGGCGGTGGCTGTCCAGCCCTCAAGCGTAGTAGAATACACCTCGCTTTGGAGCGGCTGGCGTCGCCCGCC GGACGAACCTTCTGAACTTTTCTCAAGGTTGACCTCGGATCAGGTAGGGATACCCGCTGAACTTAAGCATA TCAAAGGCGGGAAGGAAACTGGTAAGAGGTTA

gb|JQ418341.1| Diplodia corticola isolate UCROK946 18S ribosomal RNA gene, partial

sequence; internal transcribed spacer 1, 5.8S ribosomal

RNA gene, and internal transcribed spacer 2, complete sequence;

and 28S ribosomal RNA gene, partial sequence

Length $=594$

Score $=920$ bits $(498)$, Expect $=0.0$

Identities $=505 / 508(99 \%)$, Gaps $=1 / 508(0 \%)$

Strand=Plus/Plus

ESG-UK\#1-ITS4----result was Diplodia corticola (imperfect) and Botryosphaeria corticola (perfect) at Query Coverage = 95\%; $\operatorname{Max}$ identity $=99 \% ;$ expected value $=0.0$

TCAGAAGGTTCGTCCGGCGGGCGACGCCAGCCGCTCCAAAGCGAGGTGTATTCTACTACGCTTGAGGGCTG GACAGCCACCGCCGAGGTCTTTGAGGCGCGTCCGCAGAGAGGACGGCGCCCAATACCAAGCAGAGCTTGAG GGTTGTAATGACGCTCGAACAGGCATGCCCCTCGGAATGCCAAGGGGCGCAATGTGCGTTCAAAGATTCGA TGATTCACTGAATTCTGCAATTCACATTACTTATCGCATTTCGCTGCGTTCTTCATCGATGCCAGAACCAA GAGATCCGTTGTTGAAATTTTAGTTATTAACTTGTGTATCAGACGTCGACGTTCACTGACTGGAGTTTG AAGGTCCTCTGGCGGAGGGCGCGCGAGGGGGGCGGGCCCTTTTCAGGGCCTCCCCTCGCGGCGAGAGCCGC CAAAGCAACAGAGGTATGTTCACAAAGGGTGGGAGGTAACGAGCTCTCGCTCGTAGCACTCGGTAATGATC CTTCCGCAGG

gb|JQ411403.1| Diplodia corticola isolate UCROK1482 18S ribosomal RNA gene,

partial sequence; internal transcribed spacer 1, 5.8S ribosomal RNA gene, and internal transcribed spacer 2, complete sequence; and 28S ribosomal RNA gene, partial sequence Length $=586$

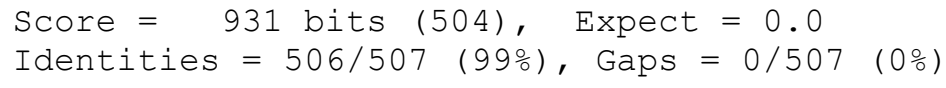

ESG-UK\#11-ITS1---result was Epicoccum nigrum at $92 \%$ of Query search but at a max identity $99 \%$ match to database sequence 
TGTCTTTTGAGTACCTTCGTTTCCTCGGCGGGTCCGCCCGCCGATTGGACAACATTCAAACCCTTTGCAGT TGCAATCAGCGTCTGAAAAAACATAATAGTTACAACTTTCAACAACGGATCTCTTGGTTCTGGCATCGATG AAGAACGCAGCGAAATGCGATAAGTAGTGTGAATTGCAGAATTCAGTGAATCATCGAATCTTTGAACGCAC ATTGCGCCCCTTGGTATTCCATGGGGCATGCCTGTTCGAGCGTCATTTGTACCTTCAAGCTCTGCTTGGTG TTGGGTGTTTGTCTCGCCTCTGCGTGTAGACTCGCCTTAAAACAATTGGCAGCCGGCGTATTGATTTCGGA GCGCAGTACATCTCGCGCTTTGCACTCATAACGACGACGTCCAAAAGTACATTTTTACACTCTTGACCTCG GATCAGGTAGgGATACCCGCTGAACTTAAgCATATCAATAAGCCGGAGGAAGAAAAATTCGgGCTGCTACC TCTTAACCCTGGGTTTT

Query ID $=$ lcl|14569

Epicoccum cf. nigrum XSCG06 18S ribosomal RNA gene, partial sequence; internal transcribed spacer 1, 5.8S ribosomal RNA gene, and internal transcribed spacer 2, complete sequence; and 28S ribosomal RNA gene, partial sequence

gb|JQ676202.1|Length: 537Number of Matches: 1

Related Information

Range 61 to 537:GenBankGraphics Next Match Previous Match

Alignment parameteres for segment \#1

Score

Expect

Identities

Gaps

Strand

881 bits (477)

0.0

$477 / 477(100 \%)$

$0 / 477(0 \%)$

Plus/Plus

\section{ESG-UK\#11-ITS4---result was Epicoccum nigrum at 92\% Query coverage; $\max$ identity $=100 \%$; expected value $=0.0$}

AAAACCCAGGgTTAAGAGGTAGCAGCCCGAATTTTTCTTCCTCCGGCTTATTGATATGCTTAAGTTCAGCG GGTATCCCTACCTGATCCGAGGTCAAGAGTGTAAAAATTACTTTTGGACGTCGTCGTTATGAGTGCAAAG CGCGAGATGTACTGCGCTCCGAAATCAATACGCCGGCTGCCAATTGTTTTAAGGCGAGTCTACACGCAGAG GCGAGACAAACACCCAACACCAAGCAGAGCTTGAAGGTACAAATGACGCTCGAACAGGCATGCCCCATGGA ATACCAAGGGGCGCAATGTGCGTTCAAAGATTCGATGATTCACTGAATTCTGCAATTCACACTACTTATCG CATTTCGCTGCGTTCTTCATCGATGCCAGAACCAAGAGATCCGTTGTTGAAAGTTGTAACTATTATGTTTT TTCAGACGCTGATTGCAACTGCAAAGGGTTTGAATGTTGTCCAATCGGCGGGCGGACCCGCCGAGGAAACG AAGGTACTCAAAAGACA

gb|JQ676202.1| Epicoccum cf. nigrum XSCG06 18S ribosomal RNA gene, partial sequence;

internal transcribed spacer 1, 5.8S ribosomal RNA gene, and internal transcribed spacer 2, complete sequence; and 28S ribosomal RNA gene, partial sequence Length $=537$

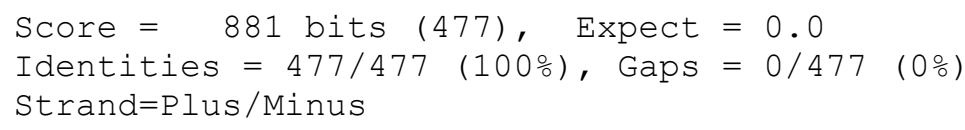


ESG-UK\#2-ITS1---- result = Paraconiothyrium sp. AND (synonymous) Microdiplodia at Query Coverage $=100 \% ;$ Max identity $=99 \%$; expected value $=0.0$

AGCTGCCGTCGGGCGGTAGAGGTAACACTTTCACGCGCCGCATGTCTGAATCCTTTTTTTACGAGCACCTT TCGTTCTCCTTCGGCGGGGCAACCTGCCGTTGGAACCTATAAAAACCTTTTTTTGCATCTAGCATTACCTG TTCTGATACAAACAATCGTTACAACTTTCAACAATGGATCTCTTGGCTCTGGCATCGATGAAGAACGCAGC GAAATGCGATAAGTAGTGTGAATTGCAGAATTCAGTGAATCATCGAATCTTTGAACGCACATTGCGCCCCT TGGTATTCCATGGGGCATGCCTGTTCGAGCGTCATCTACACCCTCAAGCTCTGCTTGGTGTTGGGCGTCTG TCCCGCCTCTGCGCGCGGACTCGCCCCAAATTCATTGGCAGCGGTCCTTGCCTCCTCTCGCGCAGCACATT GCGCTTCTCGAGGTGCGCGGCCCGCGTCCAAGAAGCAACATTACCGTCTTTGACCTCGGATCAGGTAGGGA TACCCGCTGAACTTAAGCATATCAAT

>gb|HQ999974.1| Paraconiothyrium sp. ATCC MYA-4697 18S ribosomal RNA gene, partial

sequence; internal transcribed spacer 1, 5.8S ribosomal

RNA gene, and internal transcribed spacer 2, complete sequence;

and 28S ribosomal RNA gene, partial sequence

Length $=608$

Score $=955$ bits $(517)$, Expect $=0.0$

Identities $=521 / 523$ (99\%), Gaps $=0 / 523(0 \%)$

Strand=Plus/Plus

ESG-UK\#2-ITS4---- result = Paraconiothyrium sp. AND (synonymous) Microdiplodia at Query Coverage $=100 \% ;$ Max identity $=99 \%$; expected value $=0.0$

GTTGCTTCTTGGACGCGGGCCGCGCACCTCGAGAAGCGCAATGTGCTGCGCGAGAGGAGGCAAGGACCGCT GCCAATGAATTTGGGGCGAGTCCGCGCGCAGAGGCGGGACAGACGCCCAACACCAAGCAGAGCTTGAGGGT GTAGATGACGCTCGAACAGGCATGCCCCATGGAATACCAAGGGGCGCAATGTGCGTTCAAAGATTCGATGA TTCACTGAATCTGCAATTCACACTACTTATCGCATTTCGCTGCGTTCTTCATCGATGCCAGAGCCAAGAG ATCCATTGTTGAAAGTTGTAACGATTGTTTGTATCAGAACAGGTAATGCTAGATGCAAAAAAAGGTTTTTA TAGGTTCCAACGGCAGGTTGCCCCGCCGAAGGAGAACGAAAGGTGCTCGTAAAAAAAGGATTCAGACATGC GGCGCGTGAAAGTGTTACCTCTACCGCCCGACGGCAGCTGTTGCTCCCGCCGAGGGCCGCGACCGCACCTC ATGGA

gb|HQ999974.1| Paraconiothyrium sp. ATCC MYA-4697 18S ribosomal RNA gene, partial sequence; internal transcribed spacer 1, 5.8S ribosomal RNA gene, and internal transcribed spacer 2, complete sequence; and 28S ribosomal RNA gene, partial sequence Length $=608$

Score $=917$ bits $(496)$, Expect $=0.0$ Identities $=500 / 502(99 \%)$, Gaps $=0 / 502 \quad(0 \%)$

Strand=Plus/Minus

\section{ESG-UK\#4-ITS1---- result = Bionectria and Clonostachys at Query Coverage $=100 \% ;$ Max identity $=100 \% ;$ expected value $=0.0$}

TGACATACCTATTGTTGCTTCGGCGGGATTGCCCCGGGCGCCTTGTGTGCCCCGGATCAGGCGCCCGCCTA GGAACTCTAACTCTTGTTTTATTTTGAATCTTCTGAGTAGTTTTTACAAATAAATAAAAACTTTCAACAAC GGATCTCTTGGTTCTGGCATCGATGAAGAACGCAGCGAAATGCGATAAGTAATGTGAATTGCAGAATTCAG TGAATCATCGAATCTTTGAACGCACATTGCGCCCGCCAGTATTCTGGCGGGCATGCCTGTCTGAGCGTCAT TTCAACCCTCATGCCCCTAGGGCGTGGTGTTGGGGATCGGCCAAAGCCCGCGAGGGACGGCCGGCCCCTAA 
RNA gene, and internal transcribed spacer 2, complete sequence; and 28. large subunit ribosomal RNA gene, partial sequence

Score Expect Identities Gaps Strand

1074 bits $(581) \quad 0.0 \quad 616 / 626(98 \%) \quad 10 / 626(1 \%) \quad$ Plus/Plus

ESG-DM-ITS1---- result = Ophiostoma querci; Query Coverage = $100 \% ;$ Max identity $=100 \% ;$ expected value $=0.0$

CGTACCCCGTTCTGTTCTCGTTGCTTCTGGCGGGAGGGGAGGGGCGCGTCCTTCGGGGCGTGCCTCTCTCT CCCAGGTCCCTTCGGGGCGCCCGCCAGCGGCCGCGAGCCGCCTGAACTTTTTATAAACCAGTAACGAAACG TCTGAGAAACAAACAAAAACAGCCAAAACTTTCAACAACGGATCTCTTGGCTCTGGCATCGATGAAGAACG CAGCGAAATGCGATACGTAATGCGAATTGCAGAATTCAGCGAGTCATCGAATCTTTGAACGCACATTGCGC CCGCCAGCATTCTGGCGGGCATGCCTGTCCGAGCGTCATTTCCCCCCTCAGCATACCCTTTGGGTGCGCTG GCGTTGGGGCTCCTCCGCCCTCTGTGGCGGCAGGGCCCTCAAAACCAGTGGCGGGCCCGTCTGGTTGGCTC CGAGCGCAGTACCGAACGCAAGTTCTCTCTCTCGCTCTGCAGCCCCGGTCGGTGCCCAGCCGTCAAACCGC GCAGGAGGCTCTGCTTGCAGAACCGCCTCGCATTTTTACAAGGTTGACCTCGGATCAGGTAGGATTACCCG CTGAACTTAAGC

gb|AF493243.1| Ophiostoma querci CMW2542 18S ribosomal RNA gene, partial sequence;

internal transcribed spacer 1, 5.8S ribosomal RNA gene

and internal transcribed spacer 2, complete sequence; and

28S ribosomal RNA gene, partial sequence

Length $=685$

Score $=1072$ bits $(580)$, Expect $=0.0$

Identities $=580 / 580(100 \%)$, Gaps $=0 / 580$ (0\%)

Strand=Plus/Plus

ESG-DC-ITS1---- result $=$ Phomopsis sp.; Query Coverage $=100 \% ;$ Max identity $=99 \% ;$ expected value $=0.0$

TTGTGAACTTATACCTTACTGTTGCCTCGGCGCTAGCTGGTCCCTCGGGGCCCCTCACCCTCGGGTGTTGA GACAGCCCGTCGGCGGCCAACCTAACTCTTGTTTTTACACTGAAACTCTGAGCACAAAACATAAATGAATC AAAACTTTCAACAACGGATCTCTTGGTTYCTGGCATCGATGAAGAACGCAGCGAAATGCGATAAGTAATGT GAATTGCAGAATTCAGTGAATCATCGAATCTTTGAACGCACATTGCGCCCTCTGGTATTCCGGAGGGCATG CCTGTTCGAGCGTCATTTCAACCCTCAAGCCTGGCTTGGTGATGGGGCACTGCTTCTTACCCAAGAAGCAG GCCCTGAAATTAGTGGCGAGCTCGCCAGGACCCCGAGCGCAGTAGTTAAACCCTCGCTCTGGAAGGCCCT GGCGGTGCCCTGCCGTTAAACCCCCAACTTCTGAAAATTTGACCTCGGATCAGGTAGGAATACCCGCTGAA CTTAAGCATAC

gb|HQ008926.1| Phomopsis sp. NY8054a 18S ribosomal RNA gene, partial sequence;

internal transcribed spacer 1, 5.8S ribosomal RNA gene, and

internal transcribed spacer 2, complete sequence; and $28 \mathrm{~S}$

ribosomal RNA gene, partial sequence

Length $=554$

Score $=929$ bits (503), Expect $=0.0$

Identities $=506 / 507$ (99\%), Gaps $=1 / 507$ (0\%)

Strand=Plus/Plus 
ESG-UK\#8-ITS1---- result = Cladosporium uredinicola and cladosporioides strains. Query Coverage $=100 \% ;$ Max identity $=100 \%$; expected value $=0.0$

GGGATGTTCATAACCCTTTGTTGTCCGACTCTGTTGCCTCCGGGGCGACCCTGCCTTCGGGCGGGGGCTCC GGGTGGACACTTCAAACTCTTGCGTAACTTTGCAGTCTGAGTAAACTTAATTAATAAATTAAAACTTTTAA CAACGGATCTCTTGGTTCTGGCATCGATGAAGAACGCAGCGAAATGCGATAAGTAATGTGAATTGCAGAAT TCAGTGAATCATCGAATCTTTGAACGCACATTGCGCCCCCTGGTATTCCGGGGGGCATGCCTGTTCGAGCG TCATTTCACCACTCAAGCCTCGCTTGGTATTGGGCAACGCGGTCCGCCGCGTGCCTCAAATCGACCGGCTG GGTCTTCTGTCCCCTAAGCGTTGTGGAAACTATTCGCTAAAGGGTGTTCGGGAGGCTACGCCGTAAAACAA CCCCATTTCTAAGGTTGACCTCGGATCAGGTAGGGATACCCGCTGAACTTAAGCATATC

gb|JX406571.1| Cladosporium uredinicola strain CS11673 18S ribosomal RNA gene, partial sequence; internal transcribed spacer 1, $5.8 \mathrm{~S}$ ribosomal RNA gene, and internal transcribed spacer 2, complete sequence; and 28S ribosomal RNA gene, partial sequence Length $=541$

Score $=896 \mathrm{bits}(485)$, Expect $=0.0$

Identities $=485 / 485(100 \%)$, Gaps $=0 / 485(0 \%)$

Strand=Plus/Plus

ESG-UK\# 8-ITS4---- result = Cladosporium cladoporides.; Query Coverage = $100 \% ;$ Max identity $=100 \% ;$ expected value $=0.0$

TGGGGTTGTTTTACGGCGTAGCCTCCCGAACACCCTTTAGCGAATAGTTTCCACAACGCTTAGGGGACAGA AGACCCAGCCGGTCGATTTGAGGCACGCGGCGGACCGCGTTGCCCAATACCAAGCGAGGCTTGAGTGGTGA AATGACGCTCGAACAGGCATGCCCCCCGGAATACCAGGGGGCGCAATGTGCGTTCAAAGATTCGATGATTC ACTGAATTCTGCAATTCACATTACTTATCGCATTTCGCTGCGTTCTTCATCGATGCCAGAACCAAGAGATC CGTTGTTAAAAGTTTAATTTATTAATTAAGTTTACTCAGACTGCAAAGTTACGCAAGAGTTTGAAGTGTC CACCCGGAGCCCCCGCCCGAAGGCAGGGTCGCCCCGGAGGCAACAGAGTCGGACAACAAAGGGTTATGAAC ATCCCGGTGGTTAGACCGGGGTCACTTGTAATGATCCCTCCGCAGG

gb|JQ936096.1 Cladosporium cladosporioides strain M61 18S ribosomal RNA gene, partial sequence; internal transcribed spacer 1, 5.8S ribosomal RNA gene, and internal transcribed spacer 2, complete sequence; and 28S ribosomal RNA gene, partial sequence Length $=590$

Score $=872$ bits $(472)$, Expect $=0.0$ Identities $=472 / 472(100 \%)$, Gaps $=0 / 472$ (0\%)

Strand=Plus/Minus

ESG-UK\#10-ITS1---- result = Sordariomycetes $\mathrm{sp} . ; \mathrm{Xylaria}$ sp. (possibility of Clavulinopsis; was growing quite frequently on the ground in study area); Query Coverage = 100\%; Max identity $=100 \%$; expected value $=0.0$

ACTGGTGTTTGGCTCGCCAGATATAGTCTGGTCCGTAAGCAAATCGACTGCCTGCCTGTGTAACAGGCAGG TAAGCTTTTGTCGCGGGGTCCCGGATCAACCCGGGCTAGATAGCCACTTAACTATATACTTTTTATAAAAT CTGTGAACTTACTAGGTTGGATTCTCGCCAGAGATAGTCTGGTTTCTGCTTCGCGGATCGCCTACCCGTGA CACCTGTCACAGGCAGGTATGCTTCTGCCGCGGGTCCGTAAGTAAATCATCTGTTTCGCAAGGGACGGCGA TTTACCTGTGGAAGAGGTCCTCTAACTATATTCTTAGTATAGTTTGTTCACTAGATCAAACATTCTGAATA AAAACTTAAACTAGTTAAAACTTTCAACAACGGATCTCTTGGTTCTGGCATCGATGAAGAACGCAGCGAAA 
TGCGATAAGTAATGTGAATTGCAGAATTCAGTGAATCATCGAATCTTTGAACGCACATTGCGCCCATTAGT ATTCTAGTGGGCATGCCTGTTCGAGCGTCATTTCAACCCTTAAGCCCCTGTTGCTTAGCGTTGGGAGCCTA CAGCCTGCTGTAGCTCCTTAAAGGTAGTGGCGGAGTCGGTTCACACTCTAGACGTAGTAAAATCTTTATCT CGCCTATGGATGAGCCGGCGCCTTGCCATAAAACCCCTAATCTTTCACAAGGTTGACCTCGGATCAGGTAG GAATACCCGCTGAACTTAAGCATAT

gb|JQ760139.1| Sordariomycetes sp. genotype 263 isolate FL0401

internal transcribed

spacer 1, partial sequence; $5.8 \mathrm{~S}$ ribosomal RNA gene

and internal transcribed spacer 2, complete sequence; and $28 \mathrm{~S}$

ribosomal RNA gene, partial sequence

Length $=1323$

Score $=1358$ bits $(735)$, Expect $=0.0$

Identities $=735 / 735(100 \%)$, Gaps $=0 / 735(0 \%)$

Strand=Plus/Plus

ESG-UK\#10-ITS4---- result = Sordariomycetes sp.; Xylaria

sp. (possibility of Clavulinopsis); was growing quite frequently on the ground in study area); Query Coverage $=100 \% ;$ Max identity $=100 \%$; expected value $=0.0$

GGGGTTTTATGGCAAGGCGCCGGCTCATCCATAGGCGAGATAAAGATTTTACTACGTCTAGAGTGTGAACC GACTCCGCCACTACCTTTAAGGAGCTACAGCAGGCTGTAGGCTCCCAACGCTAAGCAACAGGGGCTTAAGG GTTGAAATGACGCTCGAACAGGCATGCCCACTAGAATACTAATGGGCGCAATGTGCGTTCAAAGATTCGAT GATTCACTGAATTCTGCAATTCACATTACTTATCGCATTTCGCTGCGTTCTTCATCGATGCCAGAACCAAG AGATCCGTTGTTGAAAGTTTTAACTAGTTTAAGTTTTTATTCAGAATGTTTGATCTAGTGAACAAACTATA CTAAGAATATAGTTAGAGGACCTCTTCCACAGGTAAATCGCCGTCCCTTGCGAAACAGATGATTTACTTAC GGACCCGCGGCAGAAGCATACCTGCCTGTGACAGGTGTCACGGGTAGGCGATCCGCGAAGCAGAAACCAGA CTATCTCTGGCGAGAATCCAACCTAGTAAGTTCACAGATTTTATAAAAAGTATATAGTTAAGTGGCTATCT AGCCCGGGTTGATCCGGGACCCCGCGACAAAAGCTTACCTGCCTGTTACACAGGCAGGCAGTCGATTTGCT TACGGACCAGACTATATCTGGCGAGCCAAACACCAGTAAGTTCACAGGGGTTTAGGAGTTTTATTAAACTC TTTAATGATCCCTCCG

gb|JQ760139.1| Sordariomycetes sp. genotype 263 isolate FL0401

internal transcribed

spacer 1, partial sequence; $5.8 \mathrm{~S}$ ribosomal RNA gene

and internal transcribed spacer 2, complete sequence; and 28S

ribosomal RNA gene, partial sequence

Length $=1323$

Score $=1332$ bits $(721)$, Expect $=0.0$

Identities $=721 / 721(100 \%)$, Gaps $=0 / 721(0 \%)$

Strand=Plus/Minus

gb|HQ608148.1| Xylaria sp. TR166 internal transcribed spacer 1, partial sequence;

5.8S ribosomal RNA gene, complete sequence; and internal

transcribed spacer 2, partial sequence

Length $=727$

Score $=1299$ bits $(703)$, Expect $=0.0$

Identities $=707 / 709$ (99\%), Gaps $=0 / 709$ (0\%)

Strand=Plus/Minus

ESG-UK\#9-ITS1---- result = Pestalotiopsis caudata.; Query Coverage = $100 \% ;$ Max identity $=99 \% ;$ expected value $=0.0$ 
GTGACTTACCATTGTTGCCTCGGCAGAAGCTACCTGGTTACCTTACCTTGGAACGGCCTACCCTGTAGCGC CCTACCCTGGAACGGCCTACCCTGTAACGGCTGCCGGTGGACTACCAAACTCTTGTTATTTTATTGTAATC TGAGCGTCTTATTTTAATAAGTCAAAACTTTCAACAACGGATCTCTTGGTTCTGGCATCGATGAAGAACGC AGCGAAATGCGATAAGTAATGTGAATTGCAGAATTCAGTGAATCATCGAATCTTTGAACGCACATTGCGCC CATTAGTATTCTAGTGGGCATGCCTGTTCGAGCGTCATTTCAACCCTTAAGCCTAGCTTAGTGTTGGGAGC CTACTGCTTTTGCTAGCGGTAGCTCCTGAAATACAACGGCGGATCTGCGATATCCTCTGAGCGTAGTAATT TTTATCTCGCTTTTGACTGGAGTTGCAGCGTCTTTAGCCGCTAAACCCCCCAATTTTTAATGGTTGACCTC GGATCAGGTAGGAATACCCGCTGAACTTAAGCAT

FJ224110.1 Pestalotiopsis caudata isolate 126 18S ribosomal RNA gene, partial

sequence; internal transcribed spacer 1, 5.8S ribosomal

RNA gene, and internal transcribed spacer 2, complete sequence;

and 28S ribosomal RNA gene, partial sequence

Length $=1150$

Score $=970$ bits $(525)$, Expect $=0.0$

Identities $=530 / 532(99 \%)$, Gaps $=1 / 532(0 \%)$

Strand=Plus/Plus

ESG-UK\#9-ITS4---- result = Pestalotiopsis caudata ; Query Coverage = $100 \% ;$ Max identity $=99 \% ;$ expected value $=0.0$

CACCATTAAAAATTGGGGGGTTTAGCGGCTAAAGACGCTGCAACTCCAGTCAAAAGCGAGATAAAAATTAC TACGCTCAGAGGATATCGCAGATCCGCCGTTGTATTTCAGGAGCTACCGCTAGCAAAAGCAGTAGGCTCCC AACACTAAGCTAGGCTTAAGGGTTGAAATGACGCTCGAACAGGCATGCCCACTAGAATACTAATGGGCGCA ATGTGCGTTCAAAGATTCGATGATTCACTGAATTCTGCAATTCACATTACTTATCGCATTTCGCTGCGTTC TTCATCGATGCCAGAACCAAGAGATCCGTTGTTGAAAGTTTTGACTTATTAAAATAAGACGCTCAGATTAC AATAAAATAACAAGAGTTTGGTAGTCCACCGGCAGCCGTTACAGGGTAGGCCGTTCCAGGGTAGGGCGCTA CAGGGTAGGCCGTTCCAAGGTAAGGTAACCAGGTAGCTTCTGCCGAGGCAACAATGGTAAGTTCACATGGG TTGGGAGTTTAAAAAACTCTATAATGATCCCTCCGCA

$>$ gb|EF055188.1| Pestalotiopsis caudata strain K14DW 18S ribosomal RNA gene, partial sequence; internal transcribed spacer 1, 5.8S ribosomal RNA gene, and internal transcribed spacer 2, complete sequence; and 28S ribosomal RNA gene, partial sequence Length $=600$

Score $=977$ bits (529), Expect $=0.0$

Identities $=531 / 532(99 \%)$, Gaps $=0 / 532(0 \%)$

Strand=Plus/Minus

ESG-UK\#3-ITS1---- result = Trichoderma sp. Query Coverage = 100\%; Max identity $=99 \% ;$ expected value $=0.0$

**PCR inhibited multiple attempts. This was the best sequence though short.

CCGAGTTTACAАCTCCCAAACCCAATGTGAACGTTACCAAACTGTTGCCTCGGCGGGATCTCTGCCCCGGG TGCGTCGCAGCCCCGGACCAAGGCGCCCGCCGGAGGACCAACCAAAACTCTTATTGTATACCCCCTCGCGG GTTTTTTTATAATCTGAGCCTTCTCGGCGCCTCTCGTAGGCGTTTCGACCGAGTTTACAACTCCCAAACCC AATGTGAACGTTACCAAACTGTTGCCTCGGCGGGATCTCTGCCCCGGGTGCGTCGCAGCCCGGACCAAGCG CCCGCCGGAGGACCAACCAAAACTCTTATTGTATACCCCCTCGCGGGTTTTTTACTATCTGAGCCATCTCG GCGCCCCTCGTGGGCGTTTCGACCGAGTTTACAACTCCCAAACCCAATGTGAACGTTACCAAACTGTTGCC 
TCGGCGGGGTCACGCCCCGGGTGCGTAAAAGCCCGGAACCAGGCGCCCGCCGGAGGAACCAACCAAACTCT TTCTGTAGTCCCCTCGCGGACGTATTTCTTACAGCTCTGAGCAAAAATTCA

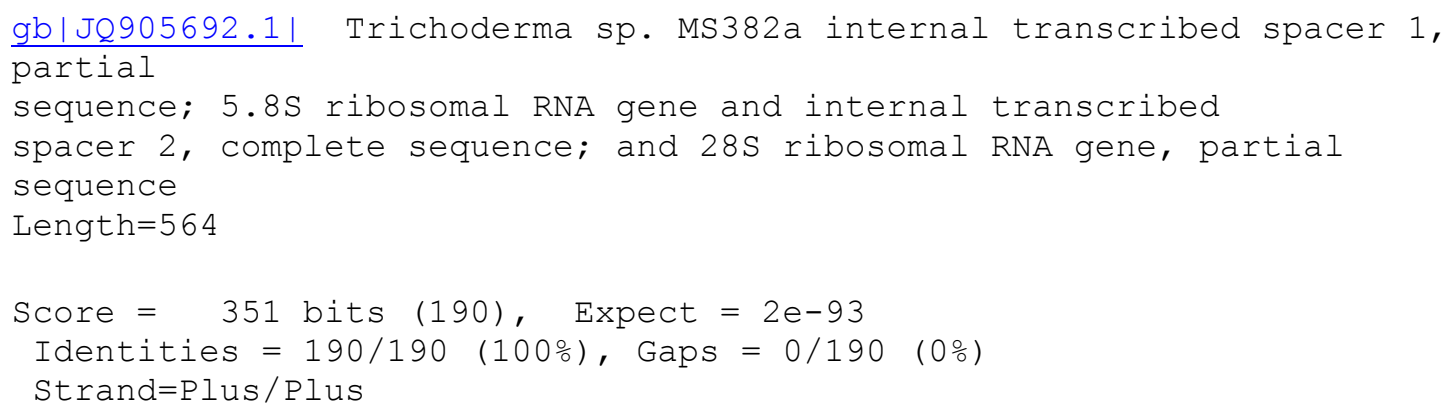

gb|GU566275.1| Mucor fragilis strain G6 18S ribosomal RNA gene, partial sequence;

internal transcribed spacer 1, 5.8S ribosomal RNA gene, and internal transcribed spacer 2, complete sequence; and 28S ribosomal RNA gene, partial sequence Length $=675$

Score $=1016$ bits $(550)$, Expect $=0.0$ Identities $=552 / 553$ (99\%), Gaps $=0 / 553(0 \%)$

Strand=Plus/Minus 


\section{LITERATURE CITED}

Alexopoulos, C.J., Mims, C.W., Blackwell, M. 1996. Introductory Mycology. John Wiley and Sons, Inc., New York. 869 pgs.

Altschul, S.F., Madden, T.L., Schäffer, A.A., Zhang, J., Zhang, Z., Miller, W. and Lipman, D.J. 1997. Gapped BLAST and PSI-BLAST: a new generation of protein database search programs. Nucleic Acids Res. 25:3389-3402

Anagnostakis, S.L. 1977. Vegetative compatibility of Endothia parasitica. Exp. Mycol. 1:306-316

Anagnostakis, S.L., Wagner, P.E. 1981. Hypovirulence, vegetative incompatibility, and the growth of cankers of chestnut blight. Phytopathology. 71:1198-1202

Anagnostakis, S.L. 1987. Chestnut Blight: The Classical Problem of an Introduced Pathogen. Mycologia. 79(1):23-37

Anderson, P. J., and H. W. Anderson. 1913. The chestnut blight fungus and a related saprophyte. The Proceedings of the PA Chestnut Blight Commission. Bulletin no. 4:1-26 pgs.

Anderson, P. J., and Babcock, D.C. 1913. Field studies on the dissemination and growth of the chestnut blight fungus. The Proceedings of the PA Chestnut Blight Commission. Bulletin no. 3:1-46 pgs.

Baird, R. 1991. Growth and stromata production of hypovirulent and virulent strains of Cryphonectria parasitica on dead Quercus rubra and Acer rubrum. Mycologia. 83:158162

Barnett, H., Hunter, B. 1998. Illustrated genera of imperfect fungi. APS Press., St. Paul, Minnesota. $4^{\text {th }}$ edition

Biraghi, A. 1953. Possible active resistance to Endothia parasitica in Castanea sativa. Rep. Congr. Int. Union For. Res. Org. 11:643-645

Braun, E.L. 1950. Deciduous Forests of Eastern North America. McGraw-Hill Book Co. Inc., New York. 1-596 pgs.

Campanile, G., Ruscelli, A., Luisi, N. 2007. Antagonistic activity of endophytic fungi towards Diplodia corticola assessed by in vitro and in planta tests. European Journal of Plant Pathology. 117(3):237-246

Choi, G., Nuss, D. 1992. Hypovirulence of chestnut blight fungus conferred by an infectious viral cDNA. Science. 257(5071, 800, ISSN: 00368075)

Cortesi, P., Milgroom, MG. 1998. Genetics of vegetative incompatibility in Cryphonectria parasitica. Appl. Environ. Microbio. 64(8):2988-2994 
Diller, J.D. 1965. Chestnut blight. USDA Forest Pest Leaflet. 94:5 pgs.

Dodds, J.A. 1980. Association of type 1 viral-like dsRNA with club-shaped particles in hypovirulent strains of Endothia parasitica. Virology. 107(1):1-12

Grente, J., Berthelay-Sauret, S. 1978. Biological control of chestnut blight in France. American Chestnut Symposium Proceeedings. (W. MacDonald, ed.), WV University Agric. Exp. Stn. And USDA.

Fulton, H. 1912. Recent notes on the chestnut bark disease. Pennsylvania Chestnut Blight Conference Report. pgs. 48-56.

Griffin, G.J. 1986. Chestnut blight and its control. Horticultural Reviews. 8:291-336

Havir, E.A., Anagnostakis, S.L. 1983. Oxalate production by virulent but not by hypovirulent strains of Endothia parasitica. Physiological Plant Pathology. 23(3):369376

Heiniger, U., Rigling, D. 1994. Biological control of chestnut blight in Europe. Ann. Rev. Phytopathology. 32:581-599

King, M. 2008. Saprophytic activity of Cryphonectria parasitica on American chestnut trees. Unpublished. West Virginia University, MacDonald, W.L. 1-21 pgs.

Kuhlman, E.G. 1978. The devastation of American chestnut by blight. Pgs. 1-3. Proceedings of the American Chestnut Symposium. MacDonald, W.L., Cech, F.C., Luchok, J., Smith, H.C. WV University Books, Morgantown, WV. 1-122 pgs.

Larena, Torres, De Cal, Liñán, Melgarejo, Domenichini, Bellini, Mandrin, Lichou, Ochoa de Eribe, Usall. 2004. Biological control of postharvest brown rot (Monilinia spp.) of peaches by field applications of Epicoccum nigrum. Biological Control. 32:305310

Lunsford, E. 1999. The American Chestnut Blight: An Agent of Biological \& Cultural Catastrophe. The American Biology Teacher. 61(8):588-593

MacDonald, W.L., Fulbright, D.W. 1991. Biological control of chestnut blight: use and limitations of transmissible hypovirulence. Plant Dis. 75:656-661

MacDonald, W.L. 1985. Hypovirulance: A potential biological control for chestnut blight. Journ. of the Amer. Ches. Found. 1(1):4

Marra, R. E., Cortesi, P. P., Bissegger, M. M., Milgroom, M. G. (2004). Mixed mating in natural populations of the chestnut blight fungus, Cryphonectria parasitica. Heredity. 93(2):189-195

McGuire, I.C., Davis, J.E., Double, M.L., MacDonald, W.L., Rauscher, J.T., McCawley, S.S., Milgroom, M.G. (2005). Heterokaryon formation and parasexual recombination between vegetatively incompatible lineages in a population of the chestnut blight fungus, Cryphonectria parasitica. Molecular Ecology. 14(12):3657-3669 
McCarroll, D.R., Thor, E. 1985. Pectolytic, cellulytic and proteolytic activities expressed by cultures of Endothia parasitica, and inhibition of these activities by components extracted from Chinese and American chestnut inner bark. Physiological Plant Pathology. 26(3):367-378

Milgroom, M.G. 1992. Comparison of genetic diversity in the chestnut blight fungus, Cryphonectria (Endothia) parasitica, from China and the United-States. Mycological Research. 96:1114-1120

Morris, T.J., Dodds, J.A. 1979. Isolation and analysis of double-stranded RNA from virus infected plant and fungal tissue. Phytopathology. 69:854-858

Murrill, W.A. 1906. A new chestnut disease. Torreya. 6:186-189

Murrill, W.A. 1906. Further remarks on a serious chestnut disease. Journal of N.Y. Botanical Gardens. 7:203-211

Nash, B.L., Stambaugh, W.J. 1982. Disease incidence, symptomology and vegetative compatibility types of Endothia parasitica on chestnut and oak hosts in North Carolina. In: Smith, H.C., MacDonald, W.L. Proceedings of the American Chestnut Symposium. West Virginia University. Pgs. 74-82

Nanako, A, Moriyama, H., Kodama, M., Arie, T., Teraoka T., Fukuhara, T. 2008. A novel mycovirus associated with four double-stranded RNAs affects host fungal growth in Alternaria alternate. Tottori. 4(101):680-8553

Newhouse, J.R. 1978. Chestnut Blight. Scientific American. 261(1):106-11

Nuss, D.L., Koltin, Y. 1990. Significance of dsRNA genetic elements in plant pathogenic fungi. Annu. Rev. Phytopathol. 28:37-58

Nuss, D.L. 2005. Hypovirulence: Mycoviruses at the fungal-plant interface. Nature Reviews Microbiology. 3(8):632-642

Powell, W.A. 1995. Vegetative incompatibility and mycelial death of Cryphonectria parasitica monitored with $\mathrm{pH}$ indicators. Mycologia. 87:738-741

Prospero, S., Condera, M., Heiniger, U., Rigling, D. 2006. Saprophytic activity and sporulation of Cryphonectria parasitica on dead chestnut wood in forests with naturally established hypovirulence. Phytopathology. 96:1337-1344

Puhalla, J.E., Anagnostakis, S. L. 1971. Endothia parasitica. Phytopatholgy. 61:169173

Rankin, W.H. 1914. Field studies on the Endothia canker of chestnut in NY state. Phytopathology. 5:45-47 
Robin, C.C., Capdevielle, X.X., Martin, M.M., Traver, C.C., Colinas, C.C. 2009. Cryphonectria parasitica vegetative compatibility type analysis of populations in southwestern France and northern Spain. Plant Pathology. 58(3): 527-535

Russell, E. 1987. Pre-light distribution of Castanea dentata (Marsh.) Borkh. Bulletin of the Torrey Bot. Club. 114:183-190

Saucier, J.R. 1945. American chestnut, an American Wood.

Marsh.Borkh. USDA.FS. 230:488-492

Shear, C.L., Stevens, N.E., Tiller, R.J. 1917. Endothia parasitica and related species. USDA Bulletin. 380:82

Shigo, A.L., Shortle, W.C., Garrett, P.W. 1977. Genetic control suggested in compartmentalization of discolored wood associated with tree wounds. Forest Science. 23(2):179-182

Shigo, A.L. 1986. A New Tree Biology. Facts, Photos, and Philosophies on Trees and Their Problems and Proper Care. Shigo and Trees, Associates; Durham, New Hampshire.

Stephenson, S.L., Adams, H.S., Lipford, M.L. 1991. The Present Distribution of Chestnut in the Upland Forest Communities of Virginia. Bulletin of the Torrey Botanical Club. 118(1):24-32

Stilwell, K.L., Wilbur, H.M., Werth, C.R., Taylor, D.R. 2003. Heterozygote advantage in the American chestnut, Castanea dentata (Fagaceae). American Journal of Botany. 90(2):207-213

Torsello, M.L. 1994. Cryphonectria parasitica cankers on scarlet oak (Quercus coccinea) in Pennsylvania. Plant Dis. 78:313-315

Welch, A., Stipanovic, A., Maynard, C., Powell, W. 2007. The effects of oxalic acid on transgenic Castanea dentata callus tissue expressing oxalate oxidase. Plant Science. 172(3):488-496

White, T., Bruns, T., Lee, S., Taylor, J. 1990. Amplification and direct sequencing of fungal ribosomal RNA genes for phylogenetics. In: PCR Protocols: a guide to methods and applications. (Innis MA, Gelfand DH, Sninsky JJ, White TJ, eds). Academic Press, New York, USA: 315-322.

West, R.F. 1988. Tolling the chestnut. American Forests. 94(10):76-77 